\title{
CARBON NANOSTRUCTURES WITH DIFFERENT DIMENSIONALITY - FROM SYNTHESIS TO APPLICATIONS
}

\author{
$\mathrm{Ph}$. D. thesis
}

\section{RÓBERT PUSKÁS}

\author{
University of Szeged
}

Department of Applied and Environmental Chemistry

Szeged

2015 
Supervisor:

Prof. Zoltán Kónya 


\section{Table of contents:}

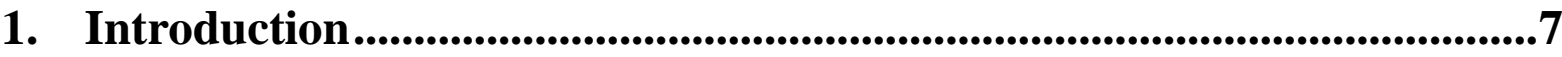

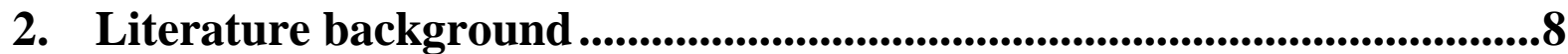

2.1. A brief history and application of carbon materials...................................... 8

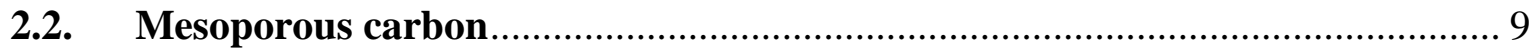

2.2.1. Introduction to mesoporous carbons ……………………………….... 9

2.2.2. Synthesis of mesoporous carbons …………….................................... 10

2.2.3. Modification of mesoporous carbons …………………………………... 13

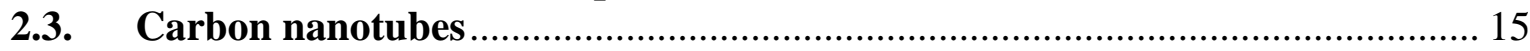

2.3.1. Various forms of carbon nanotubes …………………………………... 15

2.3.2. Preparation of carbon nanotubes …………………............................... 17

2.3.3. Purification of carbon nanotubes …………………………………...... 22

2.3.4. Modification of carbon nanotubes......................................................... 23

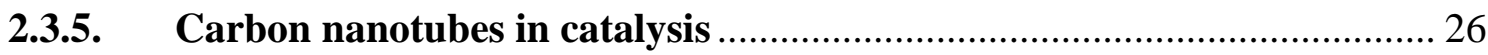

2.4. Decorating carbon materials with metal nanoparticles .................................. 28

2.5. Macroscopic carbon nanotube assemblies...……………………………….... 31

2.5.1. Introduction to macroscopic carbon nanotube structures........................ 31

2.5.2. Preparation of various carbon nanotubes assemblies ............................... 31

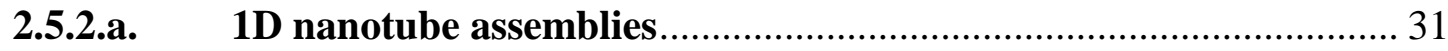

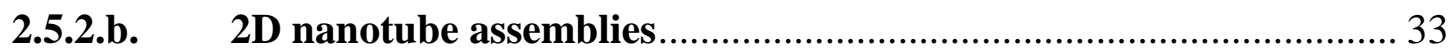

2.5.2.c. 3D nanotube assemblies.................................................................. 34

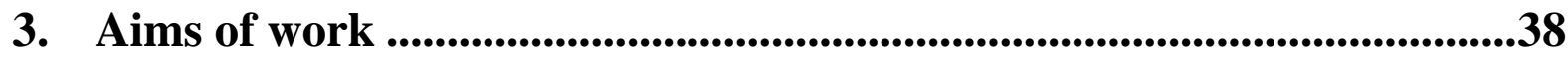

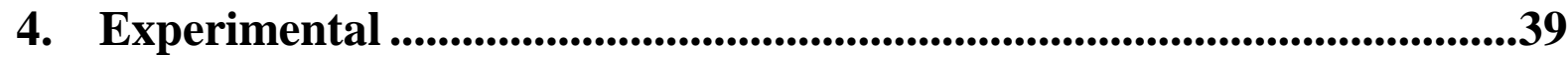

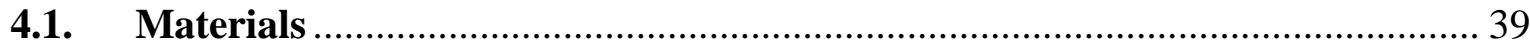

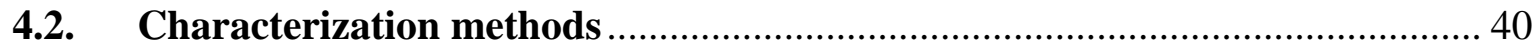

4.2.1. Transmission electron microscopy - Electron diffraction (TEM - ED)... 40

4.2.2. Scanning electron microscopy - Energy-dispersive X-ray spectroscopy (SEM - EDS)

4.2.3. X-ray diffractometry (XRD) ................................................................ 40

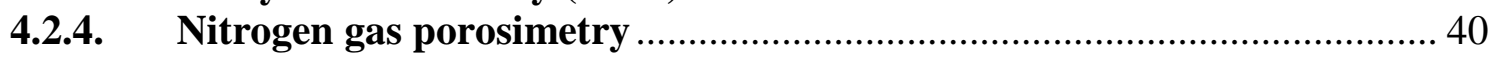

4.2.5. Thermogravimetry (TG) ……………………................................... 40

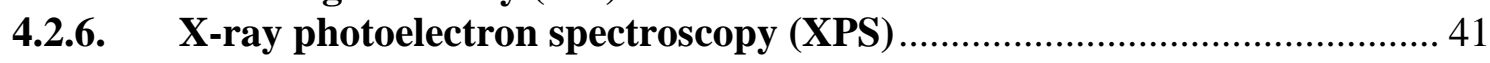

4.2.7. Dynamic mechanical analysis (DMA) ………………………………..... 41

4.2.8. Catalytic performance tests …………………...................................... 41

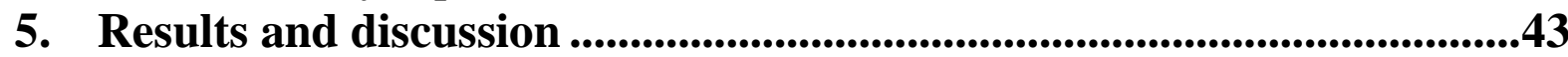

5.1. Preparation and characterization of mesoporous amorphous carbon supported Pd catalysts with tunable specific surface area and pore size ……............. 43

5.1.1. Preparation of mesoporous carbon supports ………………………...... 43

5.1.2. Characterization of silica templates and mesoporous carbon supports .. 44

5.1.3. Modification of mesoporous amorphous carbon structures with



5.1.4. Characterization of mesoporous carbon supported palladium catalysts 49

5.1.4.a. Nitrogen adsorption measurements .................................................. 49

5.1.4.b. Transmission electron microscopy ………….................................. 51

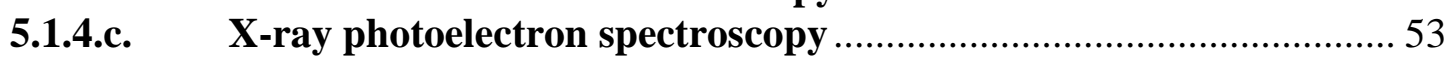

5.1.4.d. Catalytic tests ................................................................................. 54

5.2. Preparation and characterization of palladium and palladium-oxide nanoparticle modified oxidized carbon nanotubes. 
5.2.2. Characterization of palladium and palladium oxide modified "not-

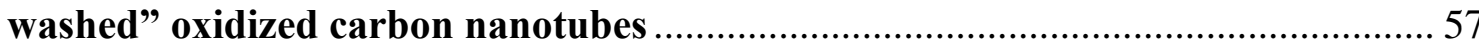

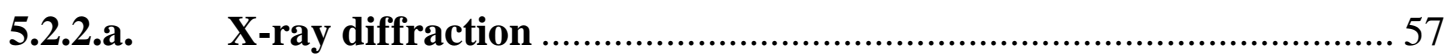

5.2.2.b. Transmission electron microscopy .............................................. 59

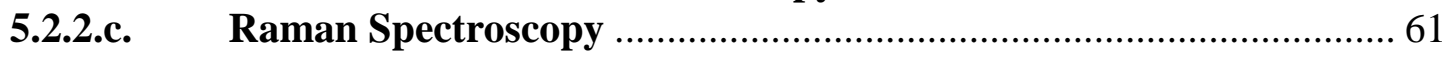

5.2.3. Characterization of palladium modified oxidized carbon nanotubes prepared with Soxhlet-extractor enhanced acetone washing. ..............................62 62

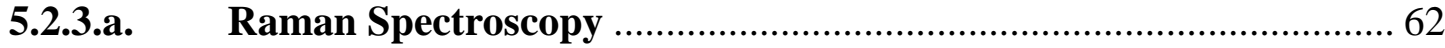

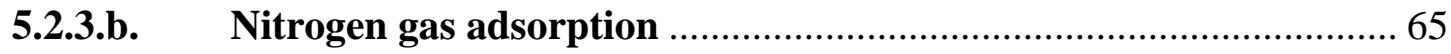

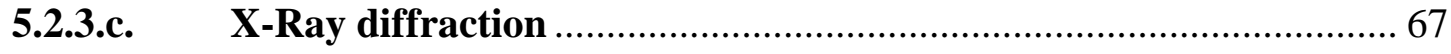

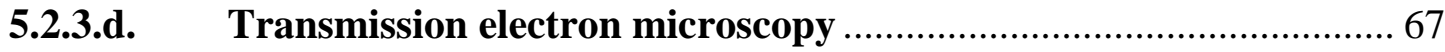

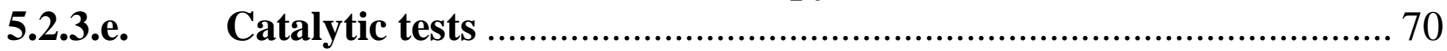

5.3. Preparation and characterization of $3 D$ structures based on functionalized

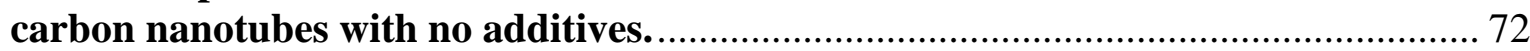

5.3.1. Preparation of 3D structures from carbon nanotubes ............................. 72

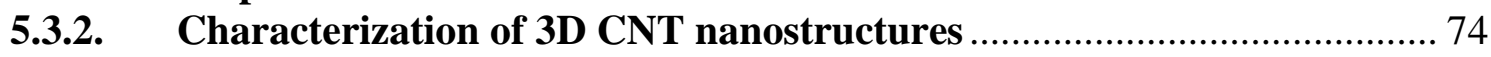

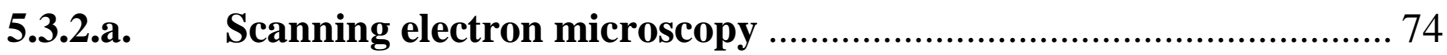

5.3.2.b. Nitrogen adsorption measurements ............................................... 76

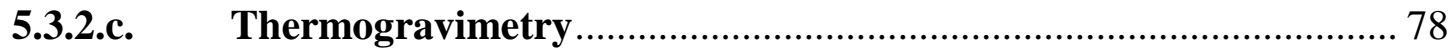

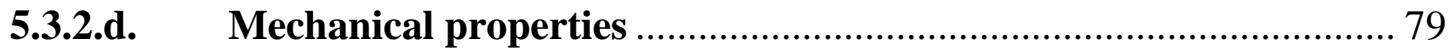

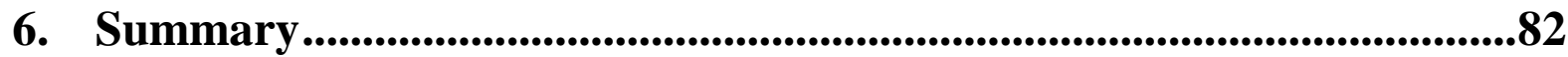

Acknowledgement ..................................................................................................86

References.....................................................................................................887 


\section{Used abbreviations}

$\mathrm{AC}$

BET

CCVD

$\mathrm{CNF}$

CNT

CNT-AW

CNT-BW

CNT-NW

$\mathrm{CO}_{2}$

CVD

CVI

dTG

DMA

DWCNT

ED

EDS

FA

FMWCNT

FWHM

GC

$\mathrm{H}_{2}$

HDP

HF

$\mathrm{HNO}_{3}$

$\mathrm{H}_{2} \mathrm{O}_{2}$

HPLC

ICDD-PDF

IUPAC

$\mathrm{KMnO}_{4}$

MC

$\mathrm{MnO}_{2}$

MWCNT
Amorphous carbon

Brunauer-Emmett-Teller method

Catalytic chemical vapor deposition

Carbon nanofiber

Carbon nanotube

"After washed" carbon nanotubes

"Before washed" carbon nanotubes

"Not washed" carbon nanotubes

Carbon dioxide

Chemical vapor deposition

Chemical vapor infiltration

Differential thermogravimetry

Dynamic Mechanical Analysis

Double-walled carbon nanotube

Electron diffraction

Energy dispersive X-ray spectroscopy

Furfuryl alcohol

Functionalized multiwalled carbon nanotubes

Full width at half maximum

Gas chromatography

Hydrogen

Homogenous Deposition Precipitation

Hydrogen fluoride

Nitric acid

Hydrogen peroxide

High performance liquid chromatography

International Center for Diffraction Data - Powder Diffraction File

International Union of Pure and Applied Chemistry

Potassium permanganate

Mesoporous Carbon

Manganese dioxide

Multiwalled carbon nanotubes 
$\mathrm{NH}_{3}$

$\mathrm{NH}_{4} \mathrm{OH}$

PTFE

PVA

$\mathrm{S}_{\mathrm{BET}}$

SEC

SEM

SWCNT

TEM

TG

TEOS

TOF

$\mathrm{V}_{\text {pore }}$

XPS

XRD
Ammonia

Ammonium hydroxide

Polytetrafluoroethylene

Polyvinyl alcohol

Specific surface area

Size exclusion chromatography

Scanning electron microscopy

Single-walled carbon nanotube

Transmission electron microscopy

Thermogravimetry

Tetraethyl orthosilicate

Turnover frequency

Specific pore volume

X-ray photoelectron spectroscopy

X-ray diffractometry 


\section{Introduction}

Nowadays is a world where new technologies are introduced almost daily, most of which are in need of energy. To mediate the ever growing energy requirements, new standards are introduced to catalyze the production of devices with higher energy efficiency. One way that has been followed for a couple of decades to increase energy efficiency is miniaturization, which is now solely driven by nanotechnology. Nanotechnology incorporates all research and processes that deal with matter in the range of 1 to $100 \mathrm{~nm}$. Since materials exhibit different characteristics in this size range than in the bulk phase, much effort has been put into developing different kinds of materials for different uses. Not only proposed uses exist, but nanomaterials are already found their way into everyday life, like computers, data storage devices, antiseptics, composite materials, self-cleaning surfaces, automobiles, industry processes, etc.

The use of nanostructures is not new in human history, as there is historical evidence that $4^{\text {th }}$ century Roman glassblowers made glass containing nanoparticles. Also, the widely acclaimed Damascus swords are known to contain nanomaterials as well. These use cases however were not deliberate, only after Richard P. Feynman's historical speech titled "There is Plenty of Room at the Bottom" held in 1959 started a real move towards nanomaterials and nanotechnology. In the following decades a number of leaps were made towards this through IBM and Bell Labs' quantum well, Taniguchi's “nanotechnology" term, Binning and Rohrer's scanning tunneling microscope, fullerenes, carbon nanotubes, graphene and well beyond.

Nanostructures are usually categorized by the number of dimensions which fit into the nano size range. If all dimensions fit into this size range then these materials are 0D or zero dimensional (quantum dots, nano clusters); if only two dimensions fit then they are 1D or one dimensional (nanotubes, nanowires, nanorods); if only one dimension fits then they are 2D or three dimensional (nanofilms); and if no dimensions fit the nano size range but the materials building up the structure are of any previous category, then they are $3 \mathrm{D}$ or three dimensional nanostructures. Such 3D nanostructures are carbon nanotube Bucky papers, aerogels or foams for example. 


\section{Literature background}

\subsection{A brief history and application of carbon materials}

Carbon materials have been used for millennia by mankind because of their high versatility. The earliest uses of charcoal started as early as 8000 years BC, which mainly consisted of burning the material for metal production. Later, in $3750 \mathrm{BC}$ charcoal was employed for the reduction of copper, manufacturing of bronze and adsorption of odorous vapors, and for various medical purposes, like mediating food poisoning cases, cleaning wounds, etc. At $5^{\text {th }}$ century BC carbons were applied for drinking water purification, taste and odor removal and disease prevention $[1,2,3,4]$.

Large scale usage however did not start until the $18^{\text {th }}$ century, where the advantageous properties of charcoal was started to be understood by a wider range of the public thanks to the work of Scheele for treatment of gases in 1773 and Lowitz for decolorification of contaminated tartaric acid solutions in 1786 . This latter property was then extensively used in the sugar industry for decolorization of raw sugar syrups. In 1822 Bussy developed the first activated carbon production procedure consisting of both thermal and chemical processes by heating blood with potash.

Industrial scale of powdered activated carbon production began in the very beginning of the $20^{\text {th }}$ century when von Ostreijko obtained two patents for chemical and thermal activation of carbon in 1900 and 1901, respectively. By utilizing the patents, "Eponit", a carbon material activated with steam and carbon dioxide entered the market in 1909. Chemical activation of sawdust with zinc chloride started on industrial scale at 1914.

The development of granular activated carbon was mainly driven by the events of World War 1, where poisonous gases were used against soldiers in the battlefields. In order to counter such attacks, gas masks were issued, where granular activated carbon was used to adsorb poisonous gases. Later, activated carbon was used in water and waste water treatment, solvent recovery, air purification and other industrial processes [5,6].

Another carbon allotrope, graphite, has also found very early usage in human history as early as $4^{\text {th }}$ millennium BC for pottery decoration [7]. In early $16^{\text {th }}$ century graphite was used for marking sheep in England, and later that century a special soft type of graphite from Borrowdale found military use, where graphite was used as a refractory material to line molds for cannonballs. This allowed the production of rounder, smoother balls which could be fired farther. In 1795 Nicolas-Jacques Conté invented the pencil, where graphite is still 
used to this day. In the past century graphite also found use in batteries, steel making, brake linings, lubricants, electronics, rubber industry and a lot of other areas [8].

Prior to the $19^{\text {th }}$ century diamond was used only for decoration and in some cases for engraving tools, but the increased availability since then allowed diamond to be used for a wide variety of things. Some notable applications include machinery manufacturing (saws, grinding wheels, boring, finishing, etc.), mineral exploration (drilling heads), ceramics and stone shaping, construction (concrete testing, masonry drilling), transportation (groove motorway concrete, bevel glass of vehicle window), electronics (slicing silicon wafers), materials science (high pressure environments, material hardness testing), optics (finishing surfaces) and a lot others [9].

Since their discovery a few decades ago, synthetic carbon allotropes like fullerenes, carbon nanotubes and graphene have been extensively studied because of their unique structure and properties. Many applications were envisioned for these materials, such as catalyst support, adsorbents, supercapacitor, field emission source, heat sink, hydrogen storage, water treatment, solar cells, electronics, medicine, actuators, batteries, etc. Despite the many potential usages, only a handful of these were realized. Carbon nanotubes for example are used as tips for atomic force microscopes, in tissue engineering for scaffolding for bone growth and as composite material for bicycles or wind turbines. Fullerenes are said to be close to be used as contrast agent for MRI or CT, or as drug or gene delivery material [10], while graphene is likely to be used in optical electronics (OLED, touchscreens) because of being highly transparent and conductive.

It can be concluded thus, that although carbon materials found use in early stages of human history dating as back as several thousand years BC, the exploitation of its real potential is still ahead of us.

\subsection{Mesoporous carbon}

\subsubsection{Introduction to mesoporous carbons}

Mesoporous carbon materials could generally be categorized as a special type of activated carbon, as both materials are built up almost entirely of carbon (other elements are also present due to functional groups), and both can be made of many different organic precursors with a high temperature carbonization process in an inert atmosphere. Also, both materials are usually amorphous in nature, but graphitic types are also possible in special cases. Furthermore, both materials exhibit exceptionally high specific surface areas, which make them suitable for a wide range of uses. The main difference between the two materials 
is the size of pores. While mesoporous materials usually exhibit narrow pore size distributions not exceeding the limits of the mesopore size range ( 2 to $50 \mathrm{~nm}$ ), activated carbon materials exhibit wide pore size distributions, where the pore size can be anywhere between the $\mathrm{nm}$ and $\mu \mathrm{m}$ range. This wide pore size distribution is due to the fact that in most cases such materials are made of organic precursors without any well-defined micro- and nanopore structure, like wood, coconut shell, bone, corn cob, apricot stone, pulp mill residues, waste tires, etc. Also, when these materials are carbonized, the pore and structure formation is not restricted by any material. Mesoporous carbons on the other hand are built on template materials with well-defined pore structures, which are finally inherited by the carbon material after the carbonization of the organic precursor. These both allow the tuning of the material for special use cases, and also enhances the physical properties of the product. Perhaps these factors were the main reasons why an immense amount of research was done in the field of mesoporous carbon production and modification.

\subsubsection{Synthesis of mesoporous carbons}

Several methods have been developed for the synthesis of mesoporous structures. Probably the most notable nowadays is the template method which was developed in the early 1980s [11,12]. This method utilizes basic steps that did not drastically change over time, thus proving the method's viability. The basic steps are as follow: the preparation of a template with controlled pore structure, impregnation of the template with some organic material, carbonization of the organics, and finally the removal of the template. These simple steps are still followed to this day, only the implementation of these steps changed. The simplicity of the method also allows the synthesis of both ordered and disordered pore structures, depending on the template, which both were extensively studied. The template method can also be categorized by the type of template used. If pre-synthesized organic or inorganic templates are used where no interactions take place between template and precursor it is called a hard template method. In this method the general structure of the resulting carbon is determined by the template only. The soft template method on the other hand relies on interactions between template and precursors. The templates are usually selfassembled organics, where molecules are organized by hydrophobic/hydrophilic interactions, hydrogen bonding, ion pairing, etc. The general structure of the resulting carbon is governed by solvents, mixing ratios and temperatures in this case. Only the hard template methods will be discussed because of their relevance in this work. 
Hard template methods can be utilized for the production of both ordered and disordered mesoporous structures. Disordered mesoporous structures can be synthesized through different approaches, which utilize copolymerization [13], anionic alumina [14,15], polymer beads [16], silica aerogels or xerogels [17,18,19] or silica spheres. Copolymerisation techniques are good when controllable pore size is needed as the ratio of precursors (TEOS and furfuryl alcohol for example) usually defines the resulting porosity of the carbon. Anionic alumina templates are suitable for the synthesis of both carbon nanotubes and amorphous carbon nanopipes. The use of polymer beads may be advantageous because it may eliminate the need for a dissolution step common for other template methods, as polymers thermally decompose while the precursor undergoes carbonization. Aluminosilicate gel templates were used to create mesoporous carbon foams with uniform pore size and opening windows. Xerogels can yield extremely high specific surface areas up to $2190 \mathrm{~m}^{2} / \mathrm{g}$, but it is important to remember that it is hard to control the final pore structure of the carbon, as the gels are usually prone to shrinkage at elevated temperatures where the carbonization happens.

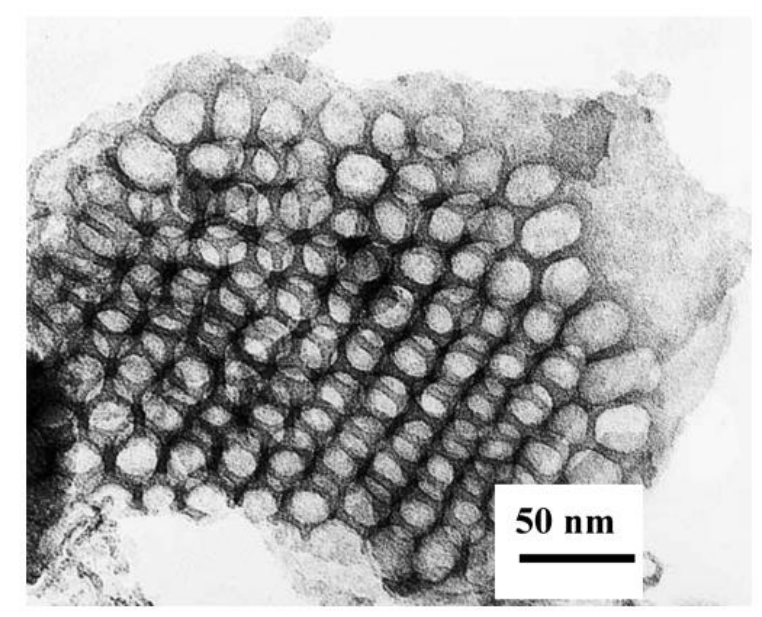

Figure 2.1. Mesocellular carbon foam templated from silica gel [19]

Colloidal silica particles were also investigated as templates, as they are probably the simplest option for reproducible carbon synthesis. Silica particles are thermally stable, easy to synthesize and cheap. Commercial silica nanoparticle sols are also available like Ludox ${ }^{\circledR}$ SM-30 or Ludox ${ }^{\circledR}$ HS-40 which are good candidates for such experiments, as carbons templated by these materials give carbon structures with very high $S_{\mathrm{BET}}\left(1512 \mathrm{~m}^{2} / \mathrm{g}\right)$ and $\mathrm{V}_{\text {pore }}\left(3.6 \mathrm{~cm}^{3} / \mathrm{g}\right)$ values [20]. The changing of precursor/silica ratio also allows a control over the resulting $\mathrm{S}_{\mathrm{BET}}\left(653-1228 \mathrm{~m}^{2} / \mathrm{g}\right)$ and $\mathrm{V}_{\text {pore }}\left(1.21-5.46 \mathrm{~cm}^{3} / \mathrm{g}\right)$ values [21]. Mesoporous carbons synthesized on silica spheres usually have spherical pores interconnected with 
smaller diameter interconnections, which are usually formed where the template particles come together. It is also possible to widen these interconnecting pores with sintering prior to impregnation, which will result in better connection between pores [22]. By choosing the proper silica spheres, bimodal mesoporous structures are also possible to create. Relatively larger, several tens of nanometers in diameter spheres are usually result in bimodal pore structure [23]. The first set of pores comes from the space liberated of nanoparticles, and the second from the space left between the spheres which were not filled with the precursor completely prior to carbonization. The bimodal distribution can also be achieved by using mixtures of ordered and colloidal silica spheres [24]. Since silica sphere templates exhibit high thermal stability, they are also suitable to be used in a relatively high temperature method, the well-known CVD method [25]. In this method there is no preliminary deposition of a precursor onto the surface of the template, but instead the precursor is thermally decomposed prior to deposition and only pure carbon meets the surface of the template.
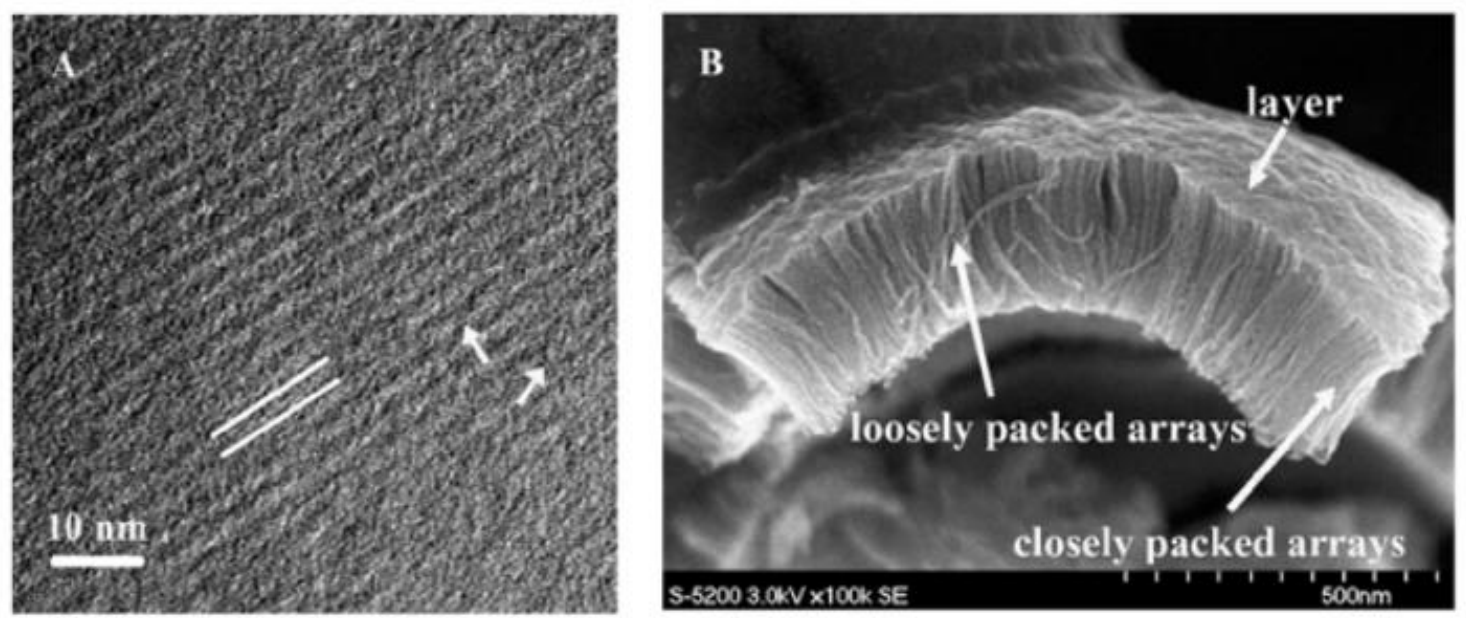

Figure 2.2. A) TEM and B) FESEM images of self-supported carbon nanowire array [29]

The first self-supported highly ordered mesoporous carbon was synthesized in 1999 on an MCM-48 aluminosilicate hard template [26]. Sucrose was impregnated in combination with sulfuric acid as carbon source, where the acid catalyzed the carbonization. The resulting material exhibited and average pore size of $3 \mathrm{~nm}$ with a unit cell size of $9 \mathrm{~nm}$, specific surface area of $1380 \mathrm{~m}^{2} / \mathrm{g}$ and a maximal total pore volume of $1.1 \mathrm{~cm}^{3} / \mathrm{g}$. Interestingly, the carbon material was not a true replica of the original template, as the cubic structures of the template and resulting material were different [27]. Studies have also shown that interconnected three-dimensional porous structures are necessary for the successful synthesis of ordered mesoporous carbons, as the hexagonally arranged one-dimensional cylindrical pores of MCM-41 for example did not yield positive results [28]. If the interconnectedness to 
the pores were restored in MCM-41, ultrathin carbon nanowire arrays could be synthesized [29]. The synthesis of ordered mesoporous carbons with bimodal pore size is also possible [30]. In this case porous nanoparticles were utilized as templates, where the smaller pores $(2.3 \mathrm{~nm})$ came from the inner pores of nanoparticles, while the larger ones $(25 \mathrm{~nm})$ from the interparticle spaces.

Different carbon sources were also used to synthesize mesoporous carbon materials, like sucrose [26], propylene [31], acetylene [32], acetonitrile [33], styrene [34], furfuryl alcohol [24], etc. While choosing the right carbon source, it must be remembered that the molecular structure of the carbon precursor can also affect the pore structure of the resulting material under certain synthesis conditions. While carbon precursors with loose molecular structures resulted in mesoporous structures complemented with micropores, fused aromatic precursors resulted in carbons with high mechanical strength and smaller specific surface area $[35,36,37]$.

\subsubsection{Modification of mesoporous carbons}

As most other carbon materials, mesoporous carbon structures can also be tuned for specific uses by modifying the physical and/or chemical characteristics. The physical characteristics such as specific surface area and total pore volume can be altered by several techniques. Probably the most basic approach is the heat treatment of material at elevated temperatures. It must be noted though, that while heat treatment usually has beneficial effects on both specific surface area and total pore volume, it also alters the surface chemistry, as acidic functional groups are thermally not that stable $[38,39]$. This explains why heat treated carbons acquire basic characteristics. The additional usage of methane and steam during heat treatments was found to alter the hydrogen and oxygen content of the resulting material. Steam treated samples contained half as much hydrogen then methane treated ones, while steam treated samples contained slightly higher amount of oxygen [40]. Apart from various heat treatment techniques, ozonation was also found to increase the specific surface area of carbons, as the reaction of ozone with the carbon structure enlarged existing pores and created new ones [41]. An increased amount of oxygen containing functional groups was also detected.

Amorphous carbon surfaces usually contain surface functional groups which can give the surface acidic, basic or neutral characteristics. It must be noted though, that even if no treatments were done, carbon materials still contain functional groups which are the result of the carbon structure itself. Amorphous carbon can be represented by randomly aligned small 
graphitic planes which are interconnected by aliphatic bindings groups. Heteroatoms are also bound to the periphery of graphitic planes which can assume the character of functional groups if found on the surface. These surface functionalities can either be modified or new functional groups can also be created. The overall surface characteristics can be altered by acidic, basic chemical treatments and/or with impregnation.

Acidic treatments mainly aim at the increase of acidic surface functional groups on the surface. Such acidic groups are carboxyl, carbonyl, hydroxyl, lactone, quinone and carboxylic anhydrate, which are created when the carbon surface is exposed to oxidizing media like nitric acid, hydrogen peroxide, etc. Such groups are also created when oxygen or ozone is used at elevated temperatures $[42,41]$. Acidic sites are usually beneficial for the uptake of metallic ions due to chelating and complex forming mechanisms. It must be noted though, that while acidic treatment increases the amount of functionalities however, the textural characteristics of the carbon usually suffer some kind of degradation, like decrease in $\mathrm{S}_{\mathrm{BET}}[43,44]$. This decrease is usually attributed to the destruction of porous structure and pore blockage (mainly microporous) caused by severe acid treatment. Other methods have also been studied in order to increase the amount of functionalities without decreasing porosity. One such promising method is treatment with oxygen plasma. The plasma did not affect microporosity, while acidic groups were also formed, thus proving the viability of the method [45]. This was explained by a hypothesis, which states that plasma does not enter into the internal structure of the carbon because of the high reactivity of mono-oxygen radicals, thus only the surface is affected and the general structure remains mostly intact [46].

Basic treatments are most commonly used to tune AC materials for organics uptake. One study suggests that $\mathrm{NaOH}$ treatment increases organics uptake, because the $\mathrm{OH}^{-}$ion reacts with existing surface functional groups, causing a major increase in phenolic groups [47]. These groups in turn change surface hydrophobicity to favor organic uptake. Ammonia treatment at high temperatures also indicate good results for organic adsorption $[48,49,50]$. It must be noted though, that while basic treatment increases organics adsorption, the uptake of metal ions severely decrease because of the reaction of the reduction of chelating groups on the surface.

Impregnation or coating of AC was also studied, where impregnation means the fine distribution of various chemicals on the surface. The main reasons for impregnation are to optimize existing properties of the material, promote synergism between impregnating agent and carbon to increase adsorption capacity, and to distribute an agent on a large surface to make it more accessible for reactions. Impregnation with tetrabutyl ammonium for example 
can increase metal uptakes several times [51]. Impregnation with metals also showed increased uptakes for certain species, such as arsenic or fluoride [52,53].

\subsection{Carbon nanotubes}

\subsubsection{Various forms of carbon nanotubes}

Carbon nanotube (CNT) is a special, artificial allotrope of carbon, which exhibits special graphite like structure made up of individual coiled graphene sheets that form cylindrical structure with diameters less than a $100 \mathrm{~nm}$ and lengths up to several millimeters. Extreme cases of nanotube length ranging from $0.5 \mathrm{~m}$ to the diameter of a phenyl group have also been reported, where the longest nanotube exhibited a length-to-diameter ratio of up to 132,000,000:1 [54]. The shortest possible carbon nanotube is in fact the organic compound cycloparaphenylene or "carbon nanohoop" (Fig. 2.3) which was has been successfully synthesized in 2008 [55].

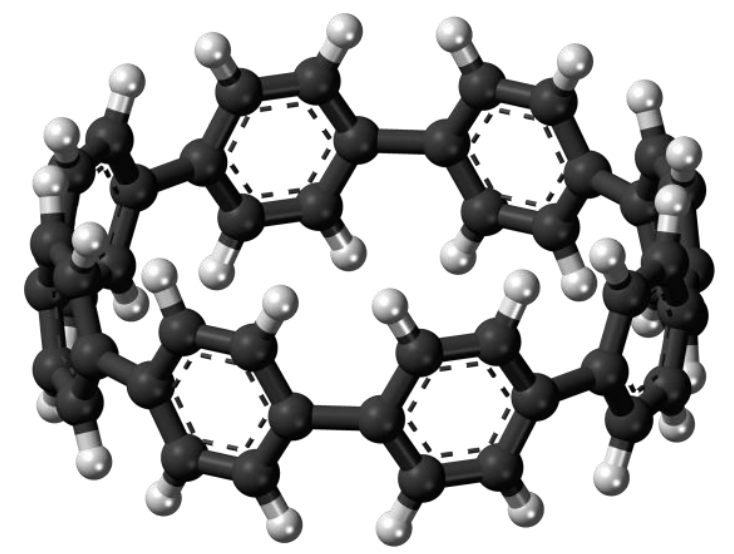

Figure 2.3: The structure of cycloparaphenylene, the shortest possible carbon nanotube

Carbon nanotubes can be classified in different ways depending on the number of walls, chirality or helicity, and wall type. According to the number of walls, single-walled, double-walled and multi-walled carbon nanotubes can be distinguished (Fig 2.4). This means that single-walled nanotubes for example have only a single layer of coiled graphitic layer or in other words the thickness of the wall is a single atom wide. Double-walled CNTs have to graphitic layers, while multi-walled almost any number above, depending on the synthesis conditions. The distance between the individual graphene walls is usually $\sim 0.34 \mathrm{~nm}$. 

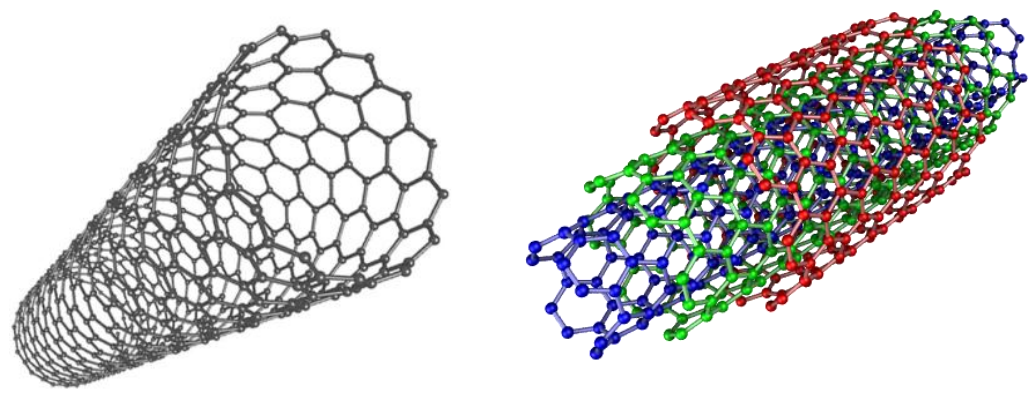

Figure 2.4. Single-walled and multi-walled carbon nanotube structures

Since graphene sheets can be rolled up in different ways, a simple method based on indices has been proposed to describe nanotubes [56]. According to this nomenclature, CNTs can be classified into two major families, the armchair and zigzag families, whose names are based on the aspect of the rolled graphene edge at the end of the tube. Both zigzag and armchair nanotubes are achiral, whereas other tubes are chiral. To understand this difference, the concept of helicity vector $\left(\mathrm{C}_{\mathrm{h}}\right)$ must be explained (Fig 2.5). $\mathrm{C}_{\mathrm{h}}$ is a vector equal to OA, where $\mathrm{O}$ is an arbitrary atom selected in a $\mathrm{C}_{6}$ ring of the graphene plane, and $\mathrm{A}$ is an equivalent carbon atom selected in another ring. OA can be decomposed into two vectors that are parallel to the graphene lattice vectors $\mathrm{a}$ and $\mathrm{b}$. By counting equivalent carbon atoms crossed by the vectors in each adjacent $\mathrm{C}_{6}$ ring, we get the indices $\mathrm{n}$ and $\mathrm{m}$, which are in this case 4 and 1 , respectively. All nanotubes are considered chiral where $n \neq m \neq 0$, and achiral where $m=0$ (zigzag) and $n=m$ (armchair).

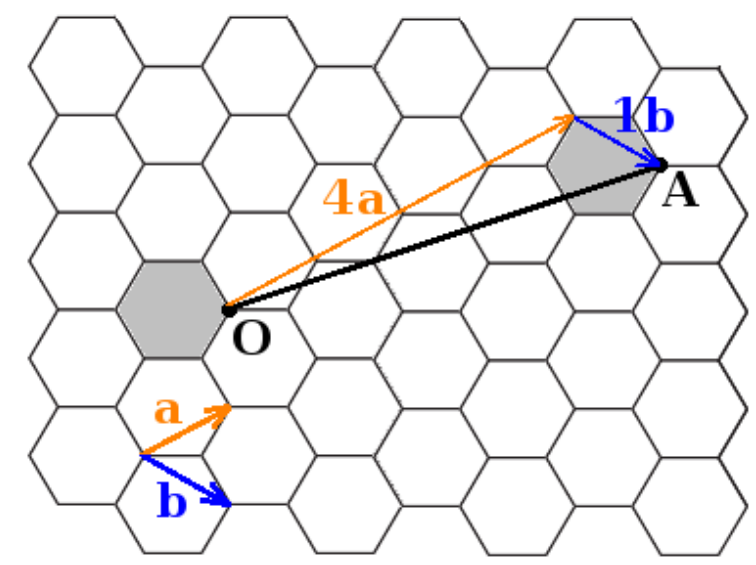

Figure 2.5. The helicity vector of CNTs

Multi-walled carbon nanotubes with several wall types have been synthesized in the past decades (Fig 2.6). Probably the most well-known is the "hollow tube" form that was 
reported in Iijima's well known article, where the axis of the graphene plane is parallel to the tube axis [57]. Herringbone structures are also possible, where graphene planes are at angle with the tube axis, forming hollow, stacked cone shaped graphene sheets [58]. This form also exists without a hollow structure, but those materials are classified as carbon nanofibers $(\mathrm{CNF})$. A transitional structure between the two aforementioned ones is the bamboo form, where the nanotube is periodically closed by a complete cone along the length of the tube forming compartments inside [59].

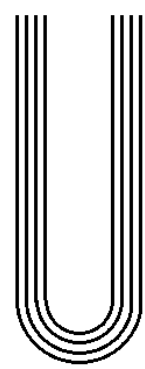

Hollow tube

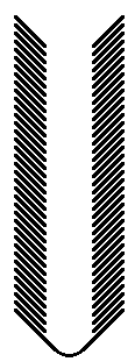

Herringbone

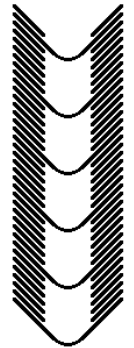

Bamboo

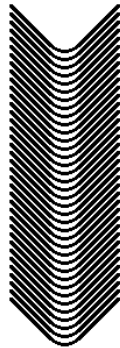

CNF

Figure 2.6. Various forms of multi-walled carbon nanotubes. A structurally closely related type of carbon nanofiber (CNF) is also shown.

\subsubsection{Preparation of carbon nanotubes}

There are four common methods to synthesize carbon nanotubes: Electric arc plasma, pulsed laser vaporization, catalytic chemical vapor deposition (CCVD) and the templatebased methods. The first two methods are less frequently used nowadays, as CCVD can be used for the preparation of both high quantity and/or quality nanotubes thanks to the relative simplicity and high customizability of the method.

Briefly both, arc and laser methods use graphite electrodes to produce CNTs. The anode or target, respectively, is atomized in high temperatures in a form of plasma reaching several thousand degrees Celsius. These methods usually generate both multi-walled and single-walled nanotubes depending on the setup. The growth mechanism for forming SWCNTs is described as a mechanism involving the atomization of the electrode at the high temperatures generated either by the arc or laser, where the atoms then move out from the hot zone forming liquid droplets with a size of several tens of nanometers, which contain both carbon species and metal catalyst. These droplets then cool down as they move out further resulting in the decrease of carbon solubility in the metal, which causes carbon to be expelled to the surface of the catalyst where SWCNTs start to grow radially [60]. This type of CNT growth produces nanotubes with relatively narrow diameter distribution centered at $1.38 \mathrm{~nm}$ and high impurity content. Fullerenes, catalyst particles, amorphous carbon, polyaromatic 
carbon soot, carbon shells are all found, thus the removal of this many types of impurities can be a challenge. Purification of nanotubes is described in the following section.

The difference between the two methods is the applied form of energy input. The arc discharge method utilizes an electric arc in a controlled environment with low pressure and inert atmosphere. During the process, the anode is consumed thus maintaining a constant distance between the electrodes is of paramount importance. This basic setup has been modified in different ways to produce different amounts or types of products by simply exchanging the catalysts (if any) and carbon source in most cases.

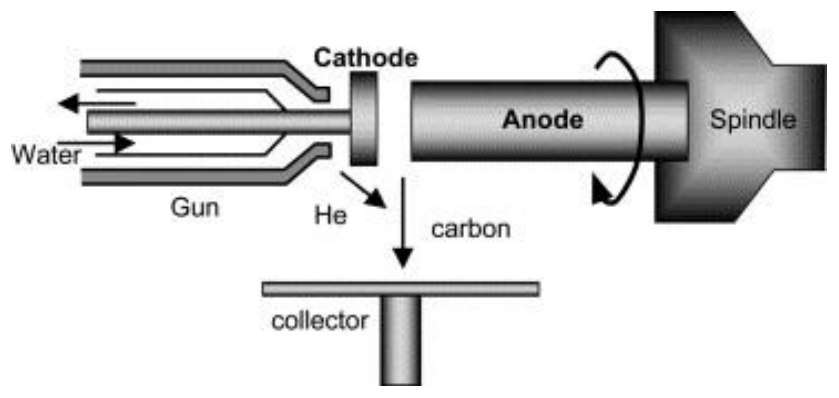

Figure 2.7. Rotating electrode setup for CNT production [61]

To simply produce a larger amount of MWCNTs, rotating pure graphite electrodes were used in 500 torr $\mathrm{He}$ atmosphere. Both hollow tube and herringbone tubes were observed as product [61]. By modifying the graphite electrode with metallic particles (1 at\% Y, 4.2 at\% Ni), a high amount of SWCNTs were produced. It must be mentioned though, that large quantities of rubbery soot, web-like structures, and other deposits were also observed [62]. If $\mathrm{Ho} / \mathrm{Ni}$ catalyst was used, web- and collar-like assemblies of SWCNT ribbons with lengths up to $10-20 \mathrm{~cm}$ were observed. The CNTs exhibiting diameters in the range of 1.30-1.64 nm formed bundles with diameters of 30-60 $\mathrm{nm}$ [63]. It can be seen, that $\mathrm{Ni}$ plays an essential role in SWCNT synthesis, however the addition of rare-earth elements can have a strong influence on the resulting product as well. The addition of other materials has also been studied, where the addition of sulphur either in the form of FeS or elemental S resulted in the formation of DWCNTs $[64,65,66]$. Sulphur can also lead to the formation of filled CNTs, or increase MWCNT diameter when PVA/Fe is used as catalyst [67].

Apart from the basic inert atmosphere environment, others have also been tested for the arc discharge method. When liquid nitrogen was tested as medium, the procedure could yield both MWCNTs [68] or SWCNTs [69]. Deionized water was also tested and was compared to liquid nitrogen as medium. It has been found that the quality of produced CNTs greatly depended on the applied voltage in liquid nitrogen, and could only obtain good quality 
nanotubes in specific parts of the sample, while water medium yielded better results. It was concluded, that despite the very low temperature of the nitrogen, it does not provide a good thermal exchange because of the violent evaporation, thus water provides a more efficient cooling. [70]. To provide even better cooling, $\mathrm{NaCl}$ solution was also tested to produce nanotubes [71].

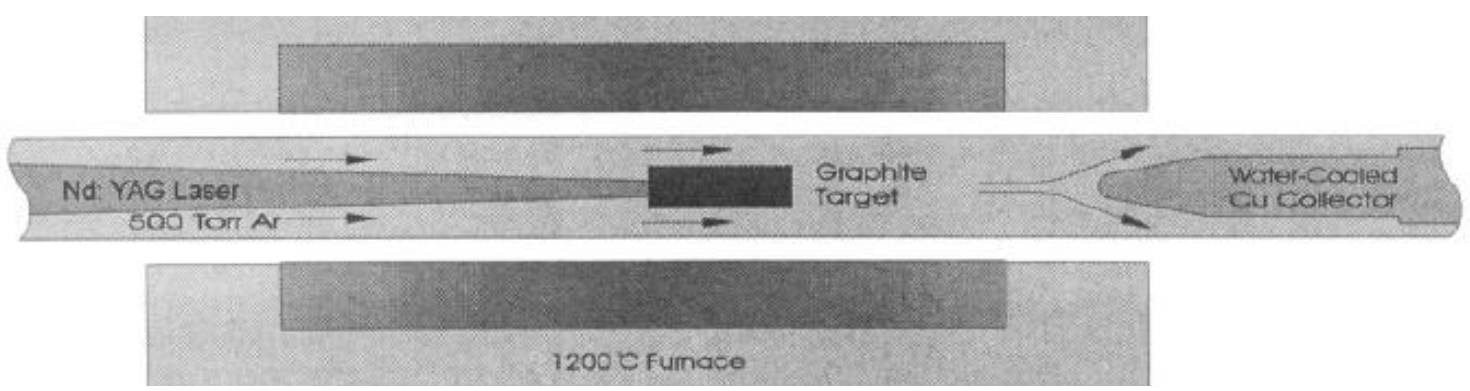

Figure 2.8. Laser ablation CNT synthesis setup [72]

The laser ablation method was first demonstrated in the middle of 90s, which utilized the same apparatus used for the production of fullerenes, metallofullerenes and MWCNTs [72]. The laser beam was scanned across the target surface in a high temperature environment to maintain a smooth surface and the resulting soot was swept out by flowing argon gas and was collected on a water cooled copper plate. This method was then modified by many other researchers to study the effect of the type of metal catalyst and ratio [73,74,75], gas type and pressure [76,77,78], furnace temperature [79,80,81] and laser parameters $[82,83,84]$. As a general summary of those findings it can be concluded, that the highest yield of CNTs can be produced with a Ni/Y catalyst, where the amount of $\mathrm{Ni}$ is higher than that of Y. Ni/Co catalyst with equal metal ratio was also found to be suitable. Gas pressures below 100 Torr did not yield significant amounts of nanotubes. The type of inert gas was also found to be important, as while CNTs grew in Ar or $\mathrm{N}_{2}$ atmosphere, no growth was observed in He. The yield and quality of nanotubes also differed if laser was operated in continuous or pulse mode. Higher laser intensity and higher furnace temperature was found to form generally larger nanotubes.

As mentioned, CCVD is probably the most widely used synthesis method for CNTs, nowadays, which can also be used for the production of boron nitride nanotubes, a close sibling of carbon nanotubes [85]. The method itself can be traced back to the 1950s at least, where a Russian team reported the synthesis of graphitic hollow structures on iron catalyst using carbon monoxide as carbon source [86]. In 1976 another team reported the synthesis of hollow graphitic structures by pyrolysing benzene [87]. After the well-known publication of Iijima [88], the reintroduction of CVD based CNT synthesis followed shortly [89]. 

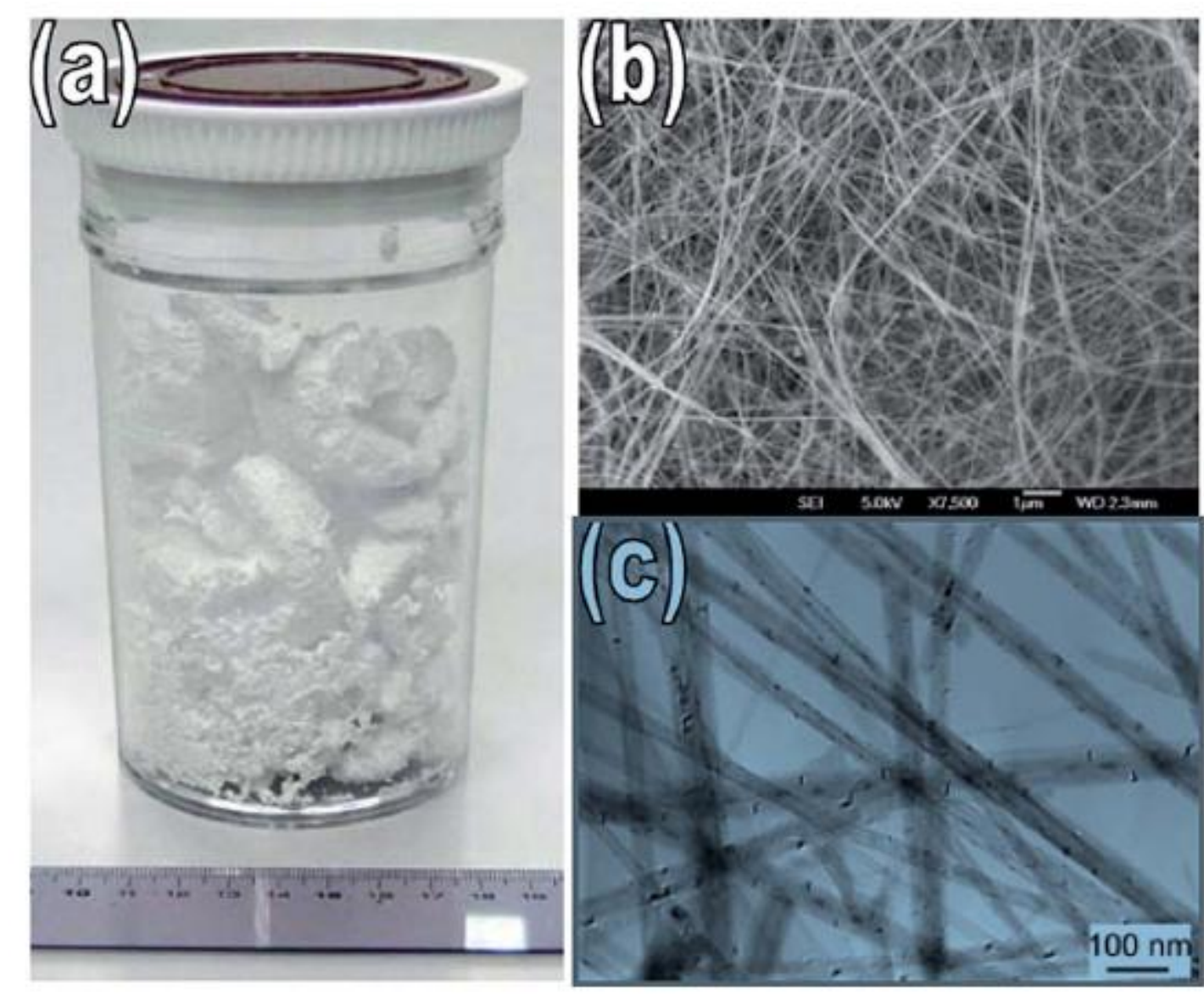

Figure 2.9.A) Photographic B) SEM and C) TEM image of boron nitride nanotubes (BNNT) [90]

The success behind the CVD method can most probably found in its general simplicity, as for a successful synthesis one basically needs only a carbon source, a metal catalyst, a tube or dome with controlled atmosphere and high enough temperatures. This simplicity also holds great possibilities for a wide range of variation, as it is clearly demonstrated by the sheer number of CVD procedures found in literature. Some of the most notable types besides the "simple" CVD are the microwave plasma-enhanced CVD [91], filament assisted CVD [92], water assisted [93] CVD, alcohol CVD [94], etc. There are some common parameters though, that remain fairly constant in all CVD types, like the gases used for producing inert atmosphere. Almost exclusively nitrogen, argon or helium is used for that purpose [95]. The type of carbon source most commonly used also shows rather small variations, as mostly light hydrocarbons, (ethylene, acetylene, methane) [96,97,98,99], some aromatics (xylene, benzene, toluene) [100,101,102], alcohols (ethanol, 2-propanol) [103,104,105,106] are used. Other types of carbon sources were also tested, like polyacrylonitrile [107,108,109] or polypyrrole $[110,111,112]$ that proved to be effective carbon sources, but the use of polymers cannot be considered widespread. 
An impressive amount of studies dealt with the effect of catalyst types on the quality and/or quantity of the CNT products. MWCNTs were grown on iron [113,114,115,116], cobalt $[117,118,119]$ and nickel $[120,121,122]$ while SWCNT production is also possible on iron $[123,124,125,126]$ cobalt $[127,128,129]$ and seldom on nickel [130]. Alloys of these metals are usually found to be more effective catalysts than standalone metals $[131,132,133,134]$. As it is clearly seen, transition metals are the most commonly used catalysts but some other metals were also studied for nanotube growth, like palladium [135]. The primary role of other metals though seems to be being a cocatalyst, which if used alone, does not show any activity from the perspective of CNT growth. Probably the most important cocatalyst is molybdenum which was added to both iron and cobalt $[136,137,138,139,140]$. Platinum, palladium and chromium were also tested as cocatalysts in conjunction with cobalt-nickel in order to lower the synthesis temperature to 773-823 K [141]. The necessity of such metals for this purpose however is questionable, as pure iron-cobalt or iron-cobaltnickel catalysts supported on aluminum are known to produce CNTs at temperatures as low as $673-723 \mathrm{~K}$ [142].

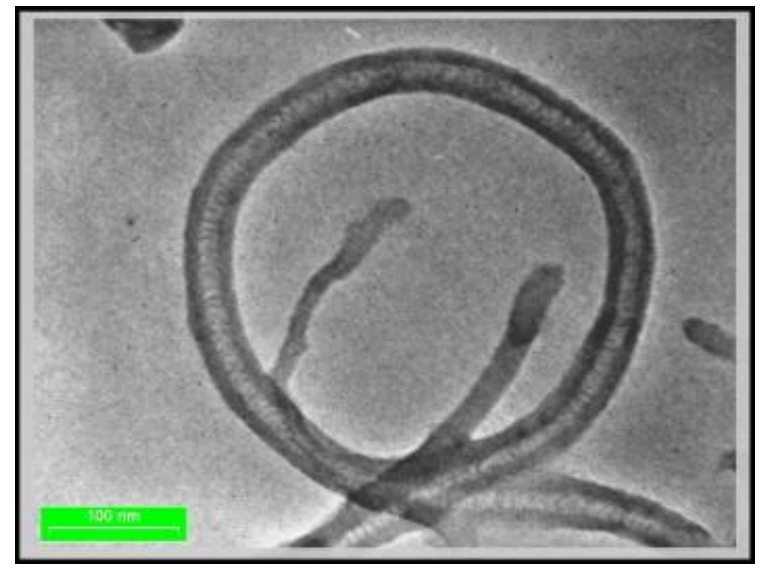

Figure 2.10. Large diameter CNT synthesized by sonochemical/hydrothermal method [144]

Alternative methods for nanotube production also exist, like the sonochemical/hydrothermal technique that has also been used to synthesize CNTs apart from other carbonaceous nanostructures, like nanorods, nanowires, nanobelts or nano-onions. This process mainly yields nanotubes with relatively large inner diameters [143,144]. Another alternative method is the production of nanotubes through electrolysis, which was proposed relatively shortly after other commonly used methods $[145,146]$. In this method the alkali or alkaline-earth metals are electroextracted from their chloride salts by putting and melting these salts $(\mathrm{LiCl}, \mathrm{LiBr} \mathrm{KCl}, \mathrm{NaCl})$ in a graphite crucible which also serves as anode. Another graphite electrode (cathode) is immersed into the molten salt to close the circuit. Metals are 
said to play and important role in some radical reactions with the help of electrical potential at the graphite surface of the cathode, where the graphite is consumed to form nanotubes.

\subsubsection{Purification of carbon nanotubes}

Several methods have been developed for nanotube purification, mostly because most current synthesis methods do not provide clean nanotubes, instead different type of impurities are also formed as byproducts. Purification methods can be divided according to their nature: chemical purification, physical purification and multi-step purification.

Chemical purification can happen in both wet and dry conditions. Wet conditions usually refer to oxidation in strong acid solutions, while the dry conditions usually refer to controlled etching in air or oxygen. Both approaches rely on the fact that amorphous carbon, carbon fragments, organics are generally more prone to oxidation then the graphitic structure of CNTs. It must be noted though, that such oxidation methods usually do not leave nanotubes intact, the tips of nanotubes for example are also affected in most cases.

For wet methods probably the most common agents used are mineral acids, as they are relatively cheap, easy to handle and no complicated procedures are necessary $[147,148,149,150,151]$. It is important to understand, that while refluxing CNTs in concentrated acid for long periods of time indeed remove most impurities, nanotubes themselves are also likely to be damaged. Refluxing in low concentration acids for shorter times on the other hand usually do not remove all impurities, thus finding the proper balance is crucial.

Oxidants other than mineral acids have also been studied for purification [152,153,154,155]. Such oxidants include potassium permanganate, hydrogen peroxide or metal cations of sufficiently high oxidation potential for example, but such methods are in many cases not used alone, as additional steps are required for the removal of solid leftovers, such as $\mathrm{MnO}_{2}$ after $\mathrm{KMnO}_{4}$ oxidation. In such cases agents not leaving byproducts behind are more preferable.

An interesting method using alkali has been established for the simultaneous purification and opening of nanotubes $[156,157]$. Sodium hydroxide was physically mixed with MWCNTs at a ratio of 3:1, which was followed by a heat treatment at $973 \mathrm{~K}$. This method seemed to remove catalyst metal particles, amorphous carbon and catalyst support but did not damage the sidewalls of nanotubes.

Microwave-assisted purification of CNTs in acid solutions have also appeared in recent years $[158,159,160,161]$. It exploits the fact that concentrated acid was found to rapidly 
absorb microwaves, allowing a quick dissolving of metal particles without the damaging of tubes. Hydrogen peroxide was also tested with the microwave-assisted technique, and it was found that $\mathrm{H}_{2} \mathrm{O}_{2}$ exhibited a higher oxidizing power towards amorphous carbon and metal particles than $\mathrm{HNO}_{3}$ over a range of temperatures, indicating that $\mathrm{H}_{2} \mathrm{O}_{2}$ is a more preferable purification agent in this case [162].

Purification of CNTs can also be conducted in gas phase, where dry or wet air, carbon dioxide, hydrogen or ammonia are commonly used. Thermal oxidation in air at moderate temperatures is effective at removing carbonaceous impurities as the presence of oxygen may result in breaking of CNT walls and weakening of the binding between entangled CNTs. As a result, amorphous content can be etched away from the walls [163,164]. It is important to choose the annealing temperature well, so that the vast majority of nanotubes are not etched away along with the impurities. Studies have found that amorphous content generally starts to decompose at $753 \mathrm{~K}$, thus temperatures much higher than this will easily result in the decomposition of nanotubes as well [165].

Other gases have also been used to purify CNTs, like carbon dioxide, hydrogen or ammonia. $\mathrm{CO}_{2}$ was found to be suitable for the selective removal of disordered carbon coming from the nanotube tips' destruction [166]. High temperature $\mathrm{H}_{2}$ can be used to remove both amorphous carbon and carbon coating on metal catalyst particles [167], and $\mathrm{NH}_{3}$ was used as a safer alternative to hydrogen [168].

There are also physical methods to purify carbon nanotubes, of which the most prominent ones are filtration, ultrasonication and chromatography. Although filtration is an easy a cheap solution, it has disadvantages, as the method is known to leave some amorphous content on the nanotube surfaces. It was also found, that this method is not well usable for low quality soot generated by arc discharge for example [169,170,171]. Sonication is an effective process for the removal of amorphous impurities, but sonicated nanotubes are also known to have a high concentration of defects. The outer graphitic layers were also found to be stripped off, leading to the thinning of nanotubes. Solvents were also found to alter the extent at which nanotubes get damaged mostly because of the difference in energy transfer capabilities of the different liquids [172,173]. High performance liquid chromatography (HPLC) [174] and size exclusion chromatography (SEC) [175,176] were also proposed for cleaning nanotubes.

\subsubsection{Modification of carbon nanotubes}


Carbon nanotubes are inherently inert chemically, which makes them unsuitable for most applications. This was probably the main driving force behind the many attempts made to modify CNTs, which modifications open up a wide range of possibilities for CNT application. There are several types of modifications available depending on the goal of the method. Nanotubes can be functionalized for attaching sidewall functional groups; doped for inserting nonisoelectronic atoms into the carbon structure (other doping types also exist); decorated with various nanoparticles, organic compounds; and filled with atoms, molecules, nanoparticles, nanowires.

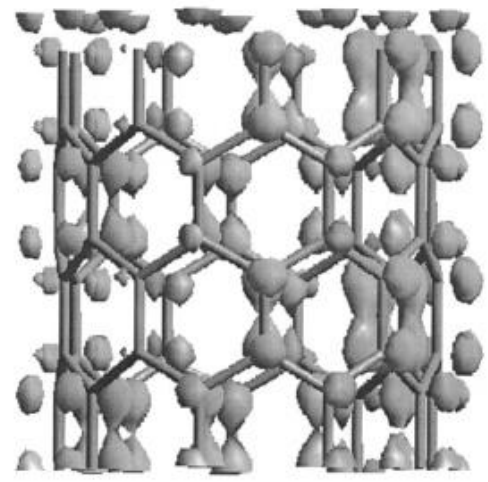

a)

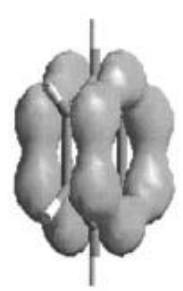

$\mathrm{C}_{6} \mathrm{H}_{6}$

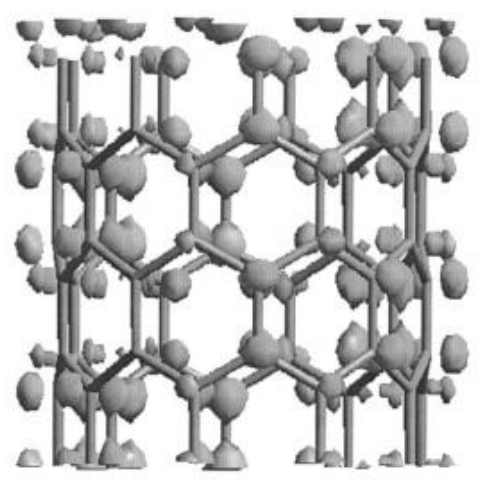

b)

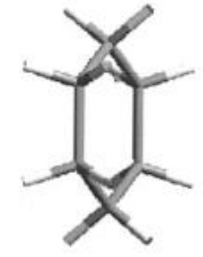

$\mathrm{C}_{6} \mathrm{H}_{12}$

Figure 2.11. Atomic structure and isosurface for the electron density distribution for the top valence band below the Fermi level. A) $\mathrm{C}_{6} \mathrm{H}_{6}$ adsorbed $(9,0)$ SWNT. B) $\mathrm{C}_{6} \mathrm{H}_{12}$ adsorbed $(9,0)$ SWNT. Coupling of the $\pi$ electrons between the molecule and the SWNT can be seen in the case of benzene adsorption [177]

Functionalization itself means, that the foreign component introduced does not form a separate phase, instead it is attached chemically or otherwise to the CNTs. It is not irreversible, defunctionalization can happen either chemically or physically. There are two basic categories of functionalization: noncovalent and covalent. Noncovalent functionalization mainly utilizes physisorption and subsequent organization of different substances on the surface of nanotubes [177]. It includes hydrophobic, $\pi-\pi$ stacking, Van der Waals and electrostatic interactions. Noncovalent functionalization has several uses, like the sorption of octadecylamine on SWCNTs for example, which allows the separation of semiconducting nanotubes from metallic ones [178]. Attaching various proteins, like cytochrome c, glucose oxidase, bovine serum albumin and many others $[179,180,181]$ is also possible. Other biomolecules were also found to attach to CNT surfaces well, which could be used to separate CNTs into fractions according to their electronic structures, diameters or helicity $[182,183,184,185]$.

Covalent functionalization itself can be divided to first generation and second generation functionalization. First generation functionalization mainly consists of attaching 
relatively simple functional groups to the surface by means of e.g. oxidation, halogenation thiolation, etc., while second generation functionalization utilizes well understood reaction routes to modify existing functional groups and attach a wide spectrum of organics, biomolecules and other materials.

Probably the most widely used first generation functionalization is the oxidation of nanotubes. Oxidation can happen in liquid or gas phase with various agents. The first method to oxidize nanotubes in gas phase was conducted in air at elevated temperatures above $973 \mathrm{~K}$, where caps and outer layers of nanotubes were found to be oxidized away [186]. A better, more controllable approach could be utilizing nitric acid vapor as a simple way to introduce oxygen containing groups to nanotube [187]. Photo-assisted oxidation of CNTs has also been investigated using oxygen [188,189]. Gas phase oxidations have an advantage of being able to omit filtration, washing and drying steps. The liquid phase oxidation however shows much greater acceptance, as it seems to be more variable allowing far greater control over the product. For this purpose different oxidizing agents, like $\mathrm{HNO}_{3}, \mathrm{H}_{2} \mathrm{SO}_{4}$, piranha $\left(\mathrm{H}_{2} \mathrm{SO}_{4} / \mathrm{H}_{2} \mathrm{O}_{2}\right.$ mixture), $\mathrm{H}_{2} \mathrm{O}_{2} \quad \mathrm{NH}_{4} \mathrm{OH} / \mathrm{H}_{2} \mathrm{O}_{2}, \quad \mathrm{HCl}$ and others have investigated [190,191,192,193,194]. It has been found that nitric acid oxidation usually resulted in a high amount of oxygen containing functional groups, while piranha oxidation was found to usually attack existing damage sites, which led to shortened nanotubes. The usage of $\mathrm{H}_{2} \mathrm{O}_{2}$ without $\mathrm{H}_{2} \mathrm{SO}_{4}$ was efficient at oxidizing nanotubes without damaging their skeleton. $\mathrm{HCl}$ usually does not yield notable amount of functional groups, thus it is better suited for purification of nanotubes.

Second generation functionalization mainly uses long-established chemical reaction routes to modify functional groups already attached to the surface of nanotubes though first generation functionalization. Fluorinated nanotubes for example can be further modified by reacting with magnesium bromide or alkyllithium precursors to generate sidewall-alkylated nanotubes [195] or with organic peroxides like lauroyl peroxide or benzoyl peroxide to produce undecyl or phenyl sidewall functionalized nanotubes [196]. The carboxylic group of oxidized CNTs can be used to create amino functional groups via the Hofmann rearrangement or Curtis rearrangement [197]. Carboxylic groups have also been subjected to silanization to attach silane groups containing epoxy end-groups, which was intended to increase the compatibility of CNTs with a polymer matrix [198]. The attachment of biomolecules have also been reported via second generation functionalization, where carboxyl groups are first converted into acyl chloride via reduction with thionyl or oxalyl chloride, then porcine pancreases lipase, amino lipase or lysine are attached via amidation of 
the previously formed acyl chloride [199,200]. The production of polypeptide-grafted CNTs is also possible by using amine-functionalized nanotubes [201]. As it is clearly seen, the attachment of a wide variety of molecules is only limited by the functional groups initially attached to the surface of CNTs and the type of reaction used. Since reactions with carboxyl groups and other simple molecules are well understood within organic chemistry, the possibilities for attaching a wide variety of organics and biomolecules to CNT surfaces are almost limitless.

\subsubsection{Carbon nanotubes in catalysis}

Carbon nanotubes have also been proposed to be used in the field of catalysis and much work has been done in this area. The reason researchers envisioned great possibilities for CNTs in this field is the resistance to acidic/basic media which allows use in harsh environments; high purity which helps avoiding self-poisoning; good thermal stability and conductivity that resist and limit catalyst damaging hot-spots; possibility to control the surface chemistry; specific metal-support interactions due to good electrical conductivity; and finally recoverability of supported metal catalysts by burning the carbonaceous support. MWCNTs are also said to be advantageous over many other support materials because of its inherent mesoporous structure, which allows better adsorption, diffusion and desorption of chemical species then found on supports with micropores. Also, the possibility to perform reactions in confined space has also been found an attractive option. Because of the aforementioned properties, carbon nanotubes have been studied to be used as support in many type of reactions, like hydrogenation, dehydrogenation, oxidation, ammonia synthesis, polymerization, hydrotreatment, etc.

The support material holds great significance, as demonstrated by several hydrogenation studies, where different supports were compared. In a hydrogenation reaction of benzene, CNTs were generally found advantageous over other supports, as with an equal loading, the nanotube supported Pd catalysts were found to yield higher conversion then zeolite or AC supported ones [202]. Also, the study revealed that if diluted Pd salt solutions were used for the support impregnation, smaller particles were obtained. Another study compared pristine and oxidized nanotubes and AC as supports, where results showed that while all samples showed similar initial conversions despite AC having several times higher specific surface area, the AC based catalyst deactivated much faster than CNT based ones, which again proves why CNTs can be better supports. [203]. The study also revealed an increase in particle diameter on spent catalysts, which can be the result of nanoparticle 
sintering during the activation process. In the hydrogenation of phenyl-acetylene CNT, AC and carbon black supported catalysts showed high conversions, but $\mathrm{Pd} / \mathrm{CNT}$ showed the highest selectivity. The AC supported catalyst also showed agglomeration of particles [204]. It can thus be seen, that there are several examples where nanotube supported catalysts show better performance from the perspective of catalyst deactivation, particle agglomeration, particle loading and selectivity. These beneficial effects are said to come from geometric, textural and electronic effects as also stated in a comparative study where $\gamma-\mathrm{Al}_{2} \mathrm{O}_{3}$ and $\mathrm{SiO}_{2}$ were compared with CNTs [205].

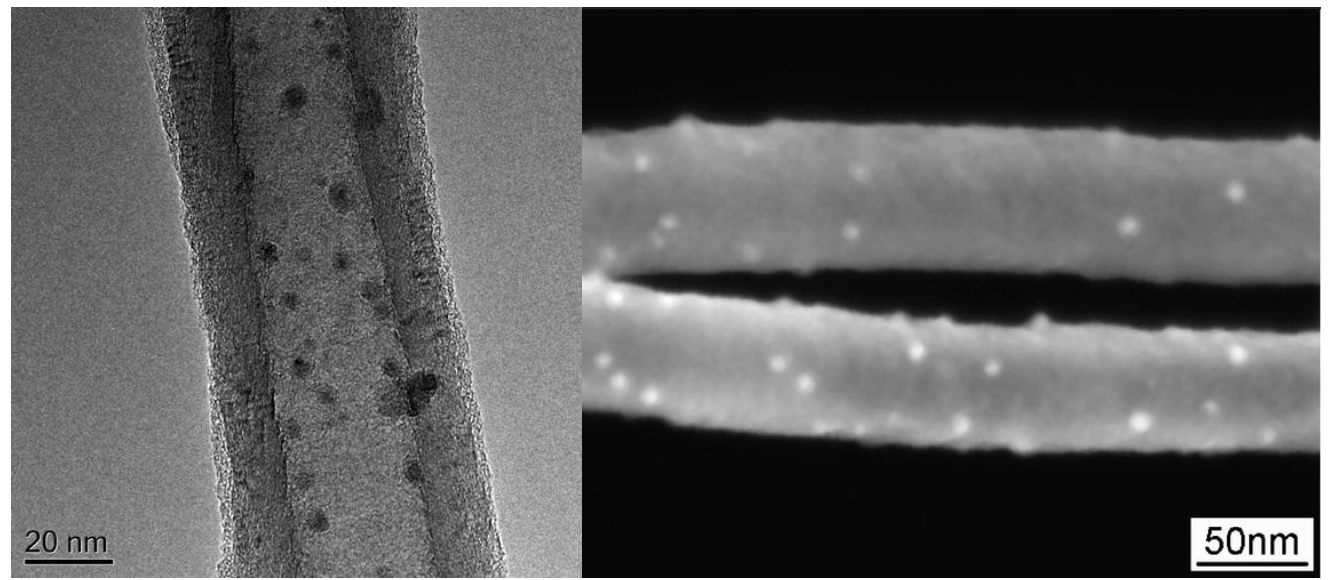

Figure 2.12. Ni nanoparticles on the inner side (left image) and outer side (right image) of carbon nanotube walls [207]

Also, confinement effects within CNTs were also studied, as these are also thought to greatly contribute to better catalyst performance on CNT supports. While normal methods, like the wet impregnation method, usually deposits nanoparticles on both the inner and outer surface of nanotubes, several methods were developed for the selective incorporation of nanoparticles to the inner side of nanotubes. Some notable ones are the reduction of organometallic precursors in supercritical carbon dioxide [206] or using surface chemistry to attract and repel nanoparticles on the inner and outer side of nanotubes, respectively [209]. By suspending nanoparticles in aqueous or organic solvents, nanoparticles can be selectively deposited on either the outer or inner surface, respectively [207]. Catalytic tests were also performed on such nanotubes. The selective hydrogenation of cinnamaldehyde for example showed an improvement in $\mathrm{C}=\mathrm{O}$ hydrogenation, rather than $\mathrm{C}=\mathrm{C}$ hydrogenation when $\mathrm{Pt}$ particles were located inside larger diameter nanotubes rather than on the outside of smaller ones [208]. In another hydrogenation reaction of cinnamaldehyde Pt-Ru nanoparticles were confined inside nanotubes, which showed similar beneficial effects. They showed higher selectivity towards the formation of cinnamyl alcohol if compared with particles deposited on the outside of the walls [209]. Pd particles were also found to show beneficial effects when 
supported on the inner nanotube walls. In a liquid phase reaction Pd particles showed better conversion then particles supported on other supports [202]. The beneficial effects of confinement on chemical reactions were also studied by theoretical studies [210,211,212]. It has been shown that chemical reactions can be influenced by confinement due to the reduced reaction volume which probably helps reactants collide more frequently as explained by the collision theory of chemical reactions. Also, the rate of reactions can be altered by several orders of magnitude within narrow ranges of pore sizes. When gas phase was compared to confined space, the endothermicity and reaction barrier were considerably reduced inside nanotubes. Also, the beneficial effect on selectivity in some reactions has been attributed to the absence of functional groups within the nanotubes.

Adsorption-desorption is also of paramount importance on carbon nanotubes from a catalytic point of view, thus much effort was made to understand these processes $[213,214,215,216,217]$. Studies showed that hydrophobic, and $\pi-\pi$ interactions are the most important factors that govern hydrocarbon adsorption onto carbon nanotubes. Hydrocarbons containing $\pi$ electrons were found to adsorb more readily onto carbon nanotubes than those without them. If nanotube surfaces were modified by functional groups, no relation was found between the adsorption of organics and the extent of functionalization. Electron donors such as PAHs were found to adsorb much stronger with the progress of functionalization, but electron acceptors such as nitroaromatics show an increase of adsorption only after mild oxidation. In an electrochemical experiment surface hydrogen was also found to be affected by functionalization. The accumulation of carboxylic groups by oxidation on carbon surfaces can increase surface conductivity by providing a mobile proton layer along the surface of carbon particles. This enhances the ability of spilled over hydrogen to diffuse to other supported metal nanoparticles more quickly, thus further lowering the inhibition effect of diffusion of reactants. It is unclear however if this effect is as strong in gaseous medium as in liquid medium. In a paper the adsorption of various C6 hydrocarbon rings were also studied on various mesoporous supports [218]. It has been found that the adsorption of the aromatics is sensitive to pressure changes on carbon nanotubes, which makes them suitable for hydrogenation reactions in more saturated feeds.

\subsection{Decorating carbon materials with metal nanoparticles}

Carbon materials can be decorated with metal nanoparticles through physical, chemical and electrochemical methods. Probably the most convenient method to deposit nanoparticles on carbon surfaces is during the synthesis of the carbon material itself. Nanoparticles such as 
$\mathrm{Co}, \mathrm{Ni}$ or Fe do not need any special procedures to be deposited, as these are usually found inside the nanotubes as a byproduct of CNT growth. Such nanoparticles are also found to be present on the surface if organometallic precursors, such as ferrocene, cobaltocene or nickelocene are used as catalyst [219,220]. These precursors are usually sprayed into the reactor where the organometallics decompose, which is followed by condensation and particle forming on all surfaces. The limitation of this technique is that depositing other metals proves very hard, and the control over particle size and metal concentration is very limited. In another vapor based technique, the laser ablation method, the concentration of metal is well tunable. Here a sheet of metal is irradiated with a high power laser under inert atmosphere and the metal vapor condenses as particles on the surface of the support [221]. Control over nanoparticle size is still limited though.

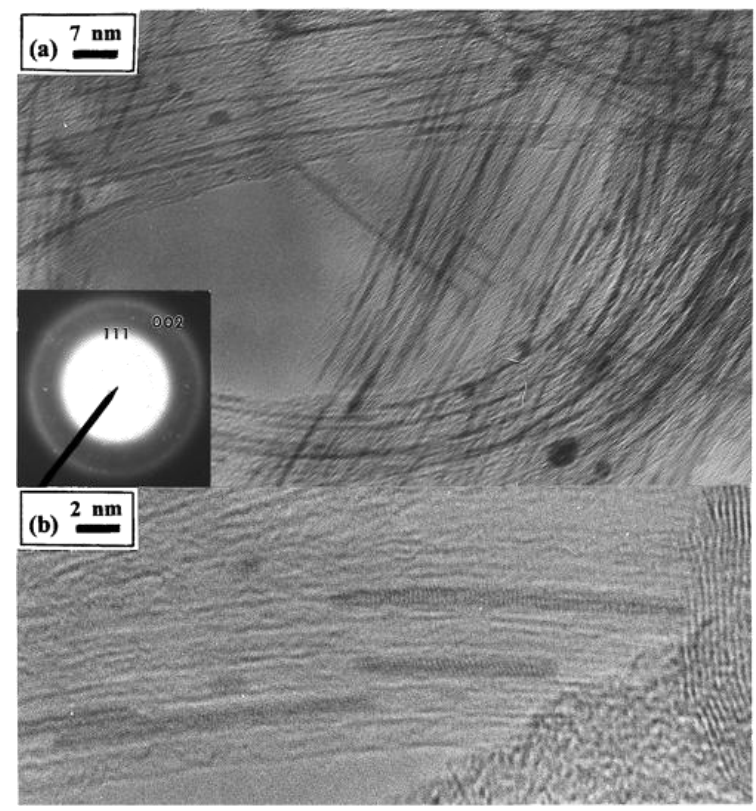

Figure 2.13. Gold nanowires found between nanotubes in an SWCNT bundle [223]

Liquid phase techniques also exist, among which the wet impregnation method is the most widely used, mostly because of its simplicity and usability with most supports and metal precursors. The wet impregnation method involves the impregnation of the support surface with a solution of metal precursor, usually some kind of a salt, which is followed by drying and calcination, where the precursor decomposes and nanoparticles form. Depending on the calcination method and precursors, a further reduction step might also be necessary to form metallic particles. This method has been used for depositing Pd, Pt, Ag, Au among others, where both nanoparticles and nanowires were made [222,223]. The nanowires only existed between SWCNTs in the bundle though, meaning that form of the wires is the result of limited space between the tubes. The homogenenous deposition precipitation (HDP) 
method is another liquid phase technique, where the precursor is reacted with another substance in the presence of the support, which results in the precipitation of a metal oxide onto the surface [224]. The oxide is then reduced to form metallic particles. This method is also known to have better nanoparticle size control then the wet impregnation method, and could be used to deposit rare earth elements such as europium or terbium among more conventional elements [225]. High concentration of nanoparticles loading is also possible. If the nanotubes are dispersed in a solution of metal salt and the salt is reduced in the liquid phase rather than gaseous phase as discussed previously, a very high loading ( $40 \mathrm{wt} \%)$ of metal is possible [226].

Another widely used liquid phase method for decoration is the polyol process [227,228,229]. This method involves the reduction of metal salts in liquid phase by the polyol. This is achieved by heating/refluxing the liquid containing both support and polyol, where the polyol decomposes which then acts as reducing agent. The size of nanoparticles depends on the amount of polyol in the mixture and the rate of reduction. For this reason the microwave assisted polyol nanoparticle coating was devised, where the heating rate, thus decomposition of the polyol is much faster than in standard heating procedures $[227,230]$. As a result, the precursor is reduced much faster and thermal gradients also become less significant, which both help nanoparticle formation by promoting instantaneous nucleation of metal clusters.

The decoration of a wide array of materials like SWCNTs [231], MWCNTs [232], carbon nanofoams [233], etc. is possible with the electroless deposition, where metal ions are reduced at a catalytically active region. The electrons needed for reduction are derived from the heterogeneous oxidation of a reducing agent, thus no external electrical current is needed, hence the name electroless deposition. The catalytically active region is not necessarily on a real catalyst particle, but surfaces with high free energy are also suitable, such as nanoparticles. The main types of electroless processes are the galvanic displacement, autocatalytic and substrate catalyzed methods [234]. At the galvanic displacement method the surface serves as both electron source and reducing agent for metal precursor reduction. At the substrate catalyzed method on the other hand the surface only serves as a catalyst for the reduction of the metal salt by a separate reducing agent. At the autocatalytic method the already deposited metal serves as catalyst for the reduction of the metal salt, the type of the surface is thus irrelevant. The latter two methods though need surface activation to start the deposition process. 


\subsection{Macroscopic carbon nanotube assemblies}

\subsubsection{Introduction to macroscopic carbon nanotube structures}

Individual carbon nanotubes can exhibit exceptional physical properties (mechanical, electrical, etc.), but exploiting these properties proved to be hard so far. Two of the major limiting factors behind the exploitation of these properties are the inertness of carbon nanotubes and that they come in a powdery form. The powder form also adds concern for safe handling the material as these powdery nanomaterials still have not well understood health effects. Although the powdered form is preferred in certain industry applications, like catalysis or nanocomposites, it is not the best form for filters, capacitors or heat sinks for example. For overcoming these problems, macroscopic functional materials need to be created such as 1D fibers, 2D sheets and 3D bulk structures, which could bring the possibility of exploiting the outstanding properties of CNTs one step closer. The major challenge behind this is to being able to assemble these individual nanomaterials into a macroscopic assembly, while still retaining all the advantageous properties. Several studies dealt already with this problem and a variety of solutions have been proposed. Basically there are three main approaches for creating bulk CNT based materials: the first is growing the material in a fashion that it forms some kind of assembly during the synthesis itself; the second where structures are assembled after the CNT synthesis mostly but not exceptionally from dispersions; and third where existing CNT structures like aligned CNT arrays or aerogels are transformed into threads, films, etc. These approaches have advantages over the other, as the first approach usually yields very high quality products, the second has much greater flexibility and third usually has the possibility to yield products on scales close or equal to semi-industrial scale. The high quality of the first method comes from very well defined, fine-tuned synthesis procedures. The greater flexibility of the second method comes from the fact that the synthesis and assembly are separate steps, which makes it possible to functionalize, clean, dope or decorate nanotubes prior to assembly. These modifications will also have an effect on the final bulk product, thus several pathways for fine tuning the properties of the assembly also becomes available. The advantage of the third method comes from the possibility of a truly continuous production of the material in the reactor itself.

\subsubsection{Preparation of various carbon nanotubes assemblies}

\subsection{2.a. 1D nanotube assemblies}

For direct fabrication of macroscopic CNT materials several attempts have been made. 1D structures such as fibers have directly been synthesized with a vertically floating CVD 
method, which produces SWCNT strands of several centimeters in length and ten to hundred micrometer thickness [235]. Although this is a direct synthetic route for CNT fiber formation, it must be noted that the final products suffer from limitations in length and reproducibility.

An intermediate way between the direct and post-synthetic assembly based approaches also exists for the preparation of nanotube assemblies, which are well suited for the creation of CNT fibers. Through these approaches fibers are created through spinning of different CNT structures, like CNT aerogels, super-aligned CNT arrays or CNT films. Spinning from CNT films usually needs an anisotropic CNT film as starting material. This method has an advantage of being able to control the diameters and twisting angles of the final product well, but has a disadvantage of being capable of producing threads with finite lengths only, as the length is basically restricted by the size of the film [236,237].

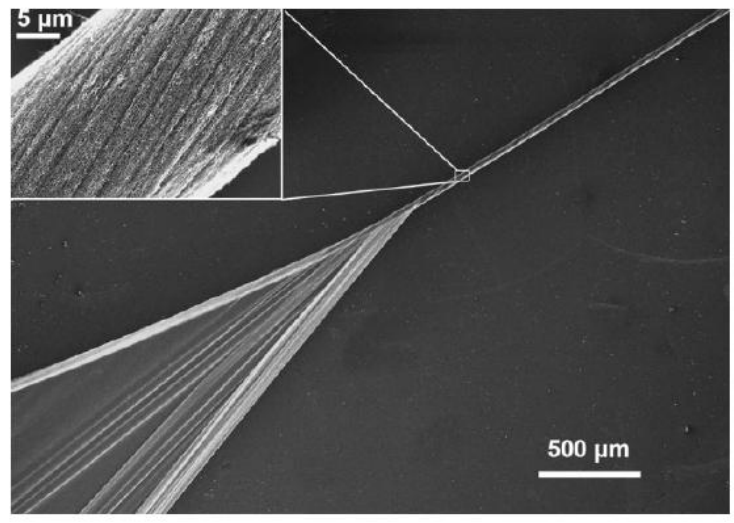

Figure 2.14. 1D nanofiber being drawn from 2DCNT film [237]

Twisting fibers from aligned CNT arrays has also been studied, and such approaches yielded fibers with electrical conductivity of $300 \mathrm{~S} / \mathrm{cm}$ and tensile strength in the range of 200-500 MPa [238]. The tensile strength could be increased up to $2 \mathrm{GPa}$ if post-spin twisting was introduced [239]. The tensile strength can also be increased by shrinking of the fiber by passing through acetone or ethanol [240]. Utilizing a laser etching method allows a fine control over the width of the resulting fibers, where the width of the fiber is determined by the width of the aligned CNT array. Fibers as thin as $10 \mu \mathrm{m}$ were created [241].

Spinning fibers from CNT aerogels hold great potential, as continuous growth of fibers can be achieved with relative ease, unlike previous methods. The key for this is to be able to rapidly synthesize high purity nanotube aerogels for spinning [242,243]. This method can result in fibers with conductivity up to $8000 \mathrm{~S} / \mathrm{cm}$ and tensile strength ranging from 0.1 to 1 GPa [244]. By controlling the synthetic conditions and spinning velocities well, multilayered CNT fibers with good macroscale uniformity and several kilometers lengths were also 
synthesized, which shows that industrial scale production of CNT fibers is now very close [245].

\subsection{2.b. 2D nanotube assemblies}

For the direct synthesis of macroscopic 2D free-standing CNT films the floatingcatalyst CVD method is equally suitable just as for the synthesis of 1D structures [246,247]. The arc-discharge method is also suitable for the direct production of CNT films but as the floating CVD method, it suffers from low product yield. Also, there are a lot of variables like the carbon source, substrate, catalyst, etc. which both can serve as a means for flexible growth control and also can overcomplicate the overall synthesis.

CNT arrays can not only be used for the creation of CNT fibers as discussed previously, but CNT films can also be drawn from them [248,249,250]. Such films exhibit large sizes up to $10 \mathrm{~cm}$ in width and $100 \mathrm{~m}$ in length where the integral nanotubes are aligned in a parallel fashion along the draw axis. The optical transmittance, electrical conductivity and light emission properties also seem to be controllable by adjusting the diameter of nanotubes and the length of the as-produced aligned CNT arrays [251].

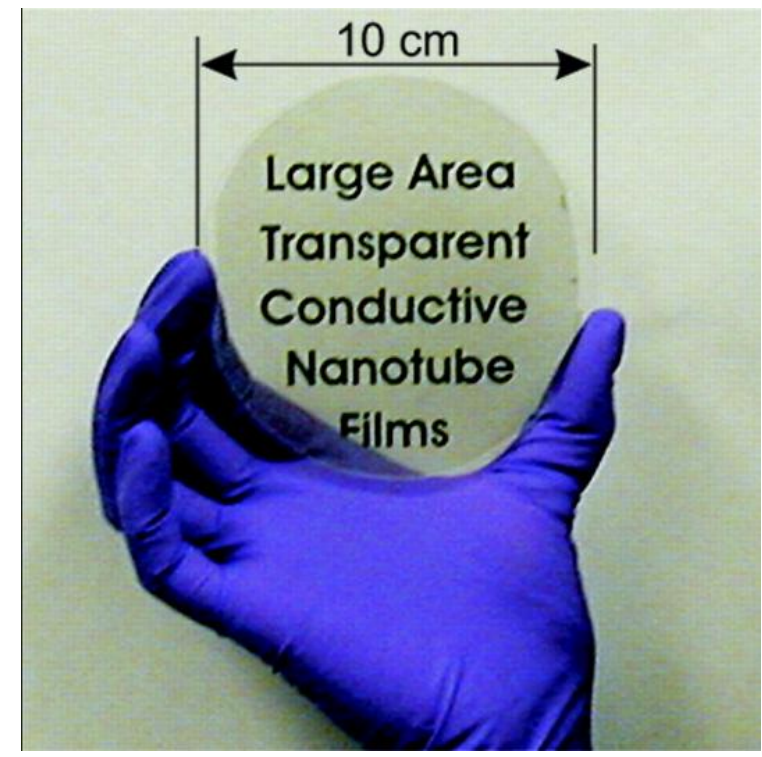

Figure 2.15. A $80 \mathrm{~nm}$ thin transparent CNT film on sapphire substrate [257]

Suspension based techniques also exist for the fabrication of CNT films such as Langmuir-Blodgett deposition [252], spin coating [253], airbrushing [254] or drop drying from solvents [255]. It must be noted, that although these processes can successfully produce CNT films, special care is needed for the transfer of the films from the original substrates. Also, very thin films with thicknesses well below the micrometer range created with these 
methods easily break or get otherwise damaged which as a result always need some kind of a support material.

Free-standing films or Bucky papers on the other hand do not need such precautions because of the more advantageous mechanical properties resulting from higher thicknesses above micrometers. Such films are easily fabricated by filtering out nanotubes from a suspension and then drying them at moderate temperatures [256]. This method allows great control over the resulting product, as the thickness of the films mostly depend on the volume and concentration of the suspension filtered. With the addition of surfactants to the suspension, ultra-thin translucent films can also be fabricated [257]. The filtration method however can only produce Bucky papers with random CNT alignment, thus the so called "domino pushing method" was devised, which allows the creation of Bucky papers with aligned CNTs [258]. This method utilizes CNT arrays as starting material, where the array is first covered with a microporous membrane, then a cylinder is rolled from one edge to the other pushing down CNTs along the axis of the cylinder movement. The resulting Bucky paper exhibited a somewhat higher conductivity of $200 \mathrm{~S} / \mathrm{m}$ over the $150 \mathrm{~S} / \mathrm{m}$ of conventional Bucky papers.

\subsection{2.c. 3D nanotube assemblies}

Three dimensional nanotube structures can mostly be classified by the alignment of CNTs in the structures. One very common type is where nanotubes are parallelly aligned thus form "CNT forests". Such CNT arrays are mostly synthesized by the CVD method, where the growth of both MWCNT based [259,260,261] and SWCNT based [262,263] forests is possible. It must be noted though, that many of the resulting forests created with various CVD methods suffer from a large amount of impurities like amorphous carbon or catalyst particles. Utilizing water vapor during the forest synthesis can reduce the amount of amorphous content, while also the activity and lifetime of the catalyst increased [264]. The proper amount of water was also investigated, and it was found that while some water vapor can increase the quality of the final product, too much water has a negative effect [265]. By optimizing all reaction conditions, including water content, a $21.7 \mathrm{~mm}$ high CNT forest was grown recently, which was a record at the time [266].

The effect of substrate was also investigated, and it was found that alumina has a crucial role for CNT super growth, where nanotube forests with millimeter scale height can be grown in very short times, like $10 \mathrm{~min}$ [267]. Weather was also found to affect nanotube forest growth. A study conducted over a 6 months period revealed that ambient humidity, 
barometric pressure and sample to sample position can greatly alter the reproducibility of experiments [268].

The safe transfer of such forests from one substrate to another can also be an important issue in certain applications, as forests supported on metallic substrates exhibit good fieldemission properties [269], while polymer supported forests can be used as flexible gas sensors [270]. Also, by growing multi-layered CNT arrays, it is possible to peel each layer off without damaging the inner structure, thus arrays with no substrate are also possible to create if needed [271].

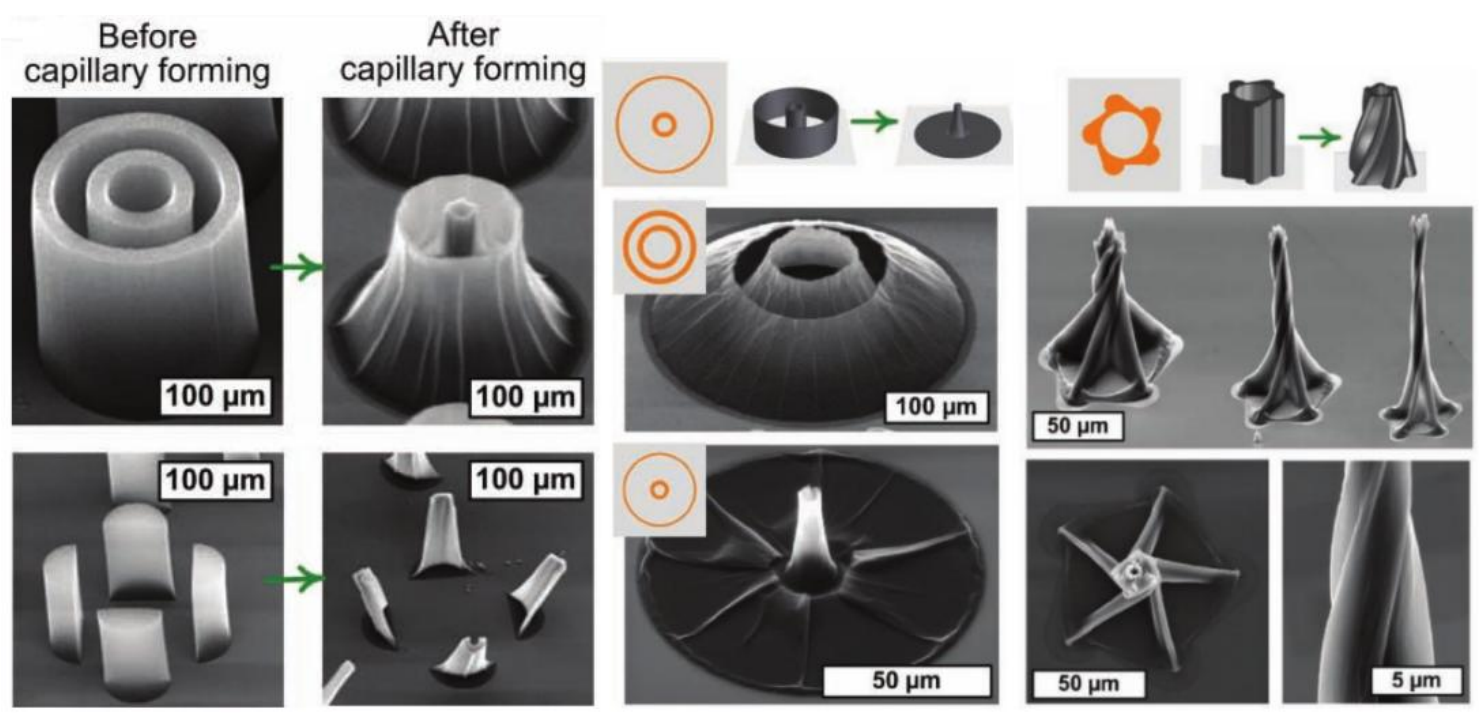

Figure 2.16. Various carbon nanotube microforms created by the "capillary forming" technique [272]

Such nanotube arrays can then be further utilized as source for other CNT macrostructures, as described previously, but it is also possible to transform them to form various three dimensional microstructures by utilizing the "capillary forming" technique [272]. This technique allows the formation of semicylinders, cylindrical forests, concentric microwells, "blooming flowers", micro-helices or thin-walled lattices. These microstructures could then be utilized as a template for materials with advanced surfaces.

Three dimensional structures with unaligned nanotubes also exist and can be prepared directly by CVD. One of such structures are CNT sponges which proved to be lightweight, reversibly and elastically deformable and exhibited high porosity [273]. By applying various post synthetic treatments, as synthesized or modified nanotubes can be transformed into three dimensional objects. One such approach is the polymerization of nanotubes under high pressure and high temperature. By applying pressures and temperatures up to $10 \mathrm{GPa}$ and $1773 \mathrm{~K}$, SWCNTs were transformed into graphite- and diamond-like microcrystalline phases, but further works seems to be needed to form continuous macroscopic crystallites with this 
method [274]. Spark plasma sintering can also be utilized for solid nanotube bulk preparation, where the resulting material exhibits low flexural strength but relatively high fracture toughness which were explained by weak bonding between nanotubes [275]. When the spark plasma sintering method was supplemented by the de-functionalization cross linking technique, it resulted in nanotube blocks with strong, covalent intertube bonding [276].

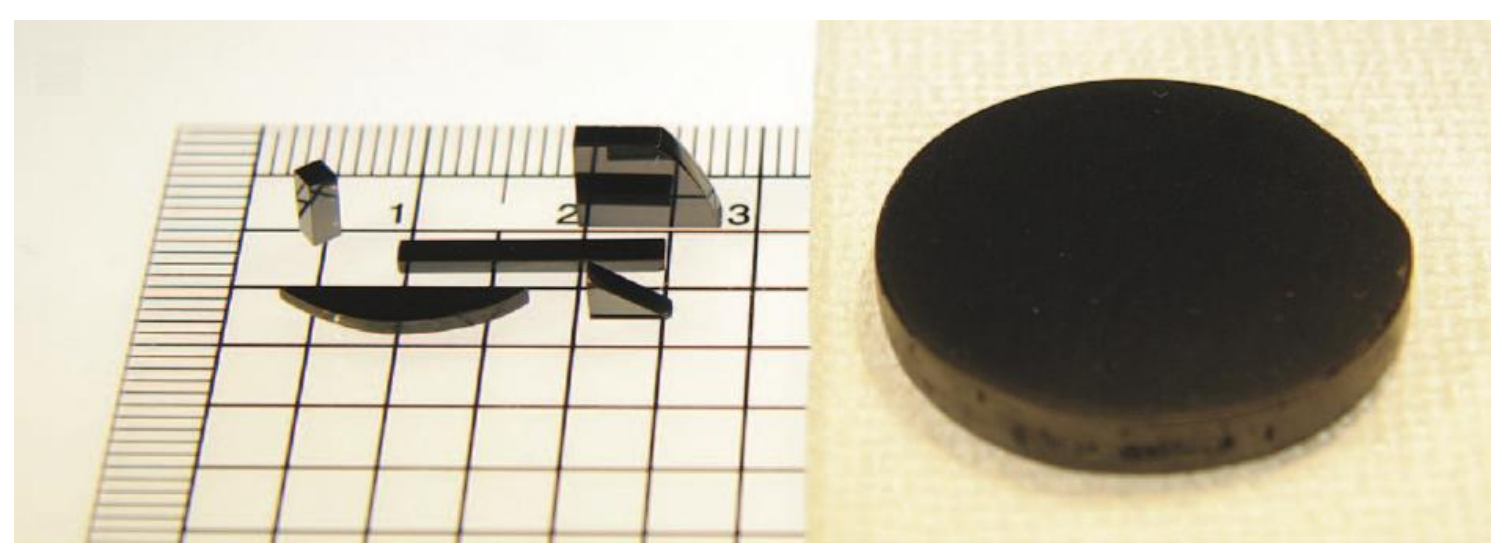

Figure 2.17. CNT blocks made using the de-fluorination technique [276]

The method used fluorinated or carboxylated nanotubes as starting material, which are subjected to high temperature $(1273 \mathrm{~K})$ and pressure $(80 \mathrm{MPa})$ in vacuo in a spark plasma sintering system. The resulting CNT blocks exhibited better mechanical properties than commercial graphite, but the conductivities were somewhat lower. De-fluorinated samples unanimously showed better results than de-carboxylated ones. The bulk density of such materials is relatively close to graphite $\left(\rho_{\mathrm{CNT}} \approx 1.3-1.4 \mathrm{~g} / \mathrm{cm}^{3}, \rho_{\text {graphite }} \approx 1.8-2.1 \mathrm{~g} / \mathrm{cm}^{3}\right.$ ), but nanotube based materials, CNT aerogels, with densities as low as $0.01-0.03 \mathrm{~g} / \mathrm{cm}^{3}$ are also possible [277,278]. Such aerogels were found to be somewhat conductive $(\sim 0.01 \mathrm{~S} / \mathrm{cm}$ or lower) and mechanically strong if the aerogels were reinforced with small amounts of PVA. Aerogels without reinforcement do not show reversible deformation or high mechanical strength, however three PVA reinforced aerogel blocks of $13 \mathrm{mg}$ total mass could support $100 \mathrm{~g}$, which is several orders of magnitude more than their own weight. Aerogels can also be reinforced with graphene, which grants these materials incredible resilience against compressive stress [279]. Such samples showed little fatigue even after 2000 or $10^{6}$ compression cycles at $1 \mathrm{~Hz}$ or $100 \mathrm{~Hz}$, respectively. 


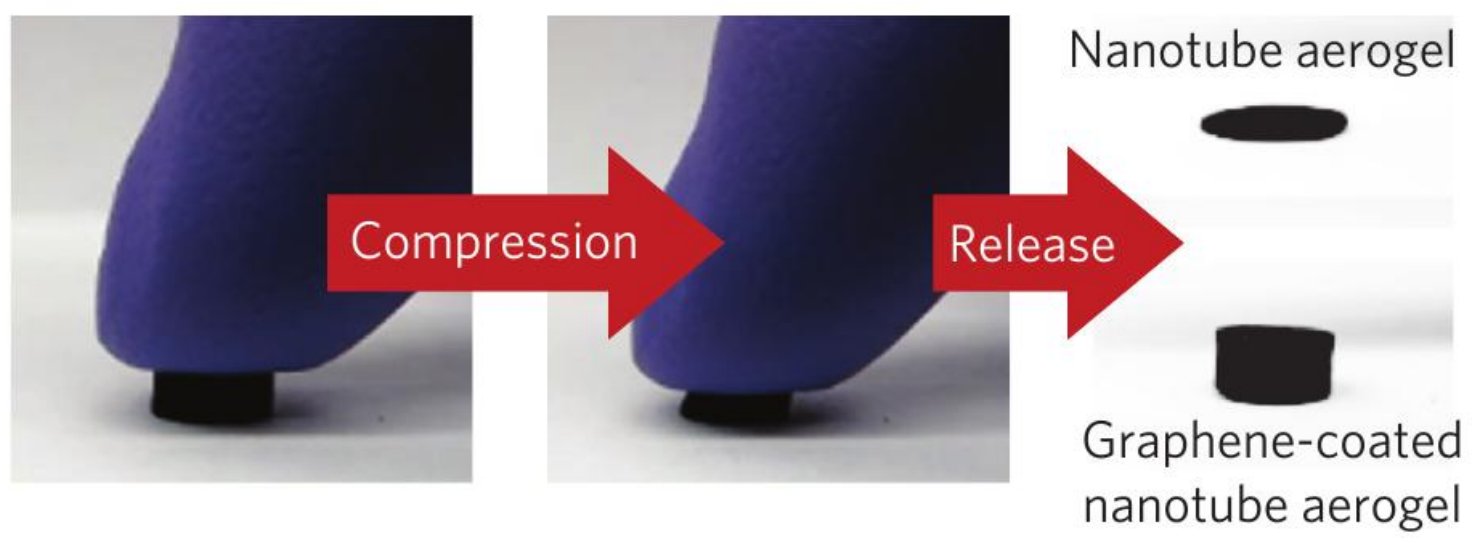

Figure 2.18. Carbon nanotube aerogels with or without graphene reinforcement [279] 


\section{Aims of work}

As discussed in the previous section, the search for carbon materials with better structure and surface environmental control has extensively been studied. For this reason many solutions were devised to control these properties, thus the aim of this work is to add more simple and reproducible methods to achieve these goals, which are also capable of circumventing the drawbacks of some existing methods. Establishing a better understanding on the effects of the much discussed nanotube functionalization was also the aim of the work, as no clear picture has been established yet. Finally, a lot of work has been done lately in devising methods to create 3D carbon nanotube based nanostructures, but unfortunately most of them prevent the fine control of the nanotubes by modification as a result of the synthetic routes themselves. This work tries to provide a means to circumvent this problem.

The main points of the work are summarized as follows:

1. To establish a method for controlling the physical properties of mesoporous carbon structures with minimal impact on wall structure arising from synthesis conditions.

2. To modify mesoporous structures with $\mathrm{Pd}$ using various techniques to reveal differences in chemical and physical properties caused by different synthetic approaches.

3. To establish an understanding of the nanoparticle size change on carbon nanotubes oxidized for different times.

4. To reveal the function of different surface moieties on carbon nanotube properties arising from both nanotube synthesis and oxidation.

5. To prepare 3D nanostructures purely from carbon nanotubes in a simple manner, where the assembly of the $3 \mathrm{D}$ structure has minimal impact on the nanotubes themselves. 


\section{Experimental}

\subsection{Materials}

For the preparation of amorphous carbon supported $\mathrm{Rh}, \mathrm{Pt}, \mathrm{Pd}$ catalysts the following materials were required:

Ludox ${ }^{\circledR}$ SM30 silica colloid - Sigma-Aldrich

Ludox ${ }^{\circledR}$ AS40 silica colloid - Sigma-Aldrich

Ludox ${ }^{\circledR}$ HS40 silica colloid - Sigma-Aldrich

Acetylene - Messer Hungaria Ltd.

Nitrogen - Messer Hungaria Ltd.

$\mathrm{NaOH}$ - Molar Chemicals Ltd., Hungary

HF solution (38\%) - Molar Chemicals Ltd., Hungary

$\operatorname{Pt}\left(\mathrm{NH}_{3}\right)_{4} \mathrm{Cl}_{2}-$ Sigma-Aldrich

Pd(II)-acetate - Sigma-Aldrich

$\mathrm{RhCl}_{3}-$ Sigma-Aldrich

For the preparation of carbon nanotube supported Pd catalysts the following materials were required:

Multiwalled carbon nanotubes (MWCNT) - SzTE TTIK AKKT

Pd-acetate - Sigma-Aldrich

cc. $\mathrm{HNO}_{3}-$ Molar Chemicals Ltd., Hungary

Acetone - Molar Chemicals Ltd., Hungary

For the catalytic tests of various carbon supported materials the following materials were used:

Cyclohexene - Sigma-Aldrich

Hydrogen - Messer Hungaria Ltd.

Nitrogen - Messer Hungaria Ltd.

Tetraethyl orthosilicate (TEOS) - Sigma-Aldrich

Ammonia solution (30\%) - Molar Chemicals Ltd., Hungary

Patosolv ${ }^{\circledR}$ - Molar Chemicals Ltd., Hungary 


\subsection{Characterization methods}

\subsubsection{Transmission electron microscopy - Electron diffraction (TEM - ED)}

Transmission electron microscopy is essential for determining the size, morphology and structure of nanomaterials. For transmission electron microscopy a FEI Tecnai G2 20 XTWIN microscope was used, operated with a tungsten cathode with an accelerating voltage of $200 \mathrm{kV}$. Samples were suspended in ethanol in order to load minute amounts of material onto lacey carbon copper grids for high resolution imaging.

Electron diffraction was used for the determination of the crystal structure of localized areas on samples in order to ascertain the homogeneity of the materials.

\subsubsection{Scanning electron microscopy - Energy-dispersive X-ray spectroscopy (SEM - EDS)}

Scanning electron microscopy was used for the determination of the general structure and morphology of 3D materials fabricated from carbon nanotubes. For these measurements a Hitachi S-4700 microscope was used equipped with a field emission gun operated with accelerating voltages of $5-30 \mathrm{kV}$.

Energy dispersive X-ray spectroscopy was used for the determination of the elemental composition of samples. This technique was especially useful for the determination of metal content in catalysts.

\subsubsection{X-ray diffractometry (XRD)}

The crystal structure of samples was determined by powder X-ray diffractometry with a Rigaku Miniflex II diffractometer using $\mathrm{CuK} \alpha(\lambda=1.5418 \AA \AA)$ radiation. Samples were loaded onto a Si singlecrystal holder cut specifically not to give signals. Peaks were identified by comparing diffractograms with ICDD-PDF cards.

\subsubsection{Nitrogen gas porosimetry}

Nitrogen adsorption isotherms were collected with a Quantachrome NOVA 3200 instrument at $-196{ }^{\circ} \mathrm{C}(77 \mathrm{~K})$. Specific surface area and pore size distribution data were calculated by the well-known BET and BJH equations using adsorption and desorption isotherms, respectively.

\subsubsection{Thermogravimetry (TG)}


Thermal stability of samples was determined by thermogravimetry, using a TA Instruments TGA Q500 and Setaram Labsys instruments for 3D CNT structures and powder samples, respectively. Differential weight loss vs. temperature (differential thermogravimetric - dTG) curves were also calculated from weight loss vs. temperature (thermogravimetric - TG) curves. Measurements were conducted in both inert (nitrogen) and oxidative (air) atmospheres.

\subsubsection{X-ray photoelectron spectroscopy (XPS)}

X-ray photoelectron spectra were collected using a SPECS instrument equipped with a PHOIBOS 150 MCD 9 hemispherical electron energy analyzer operated in the FAT mode. The excitation source was the $K_{\alpha}$ radiation of an aluminum anode $(h v=1486.6 \mathrm{eV})$. The Xray gun was operated at $210 \mathrm{~W}(14 \mathrm{kV}, 15 \mathrm{~mA})$. The pass energy was set to $20 \mathrm{eV}$, the step size was $25 \mathrm{meV}$ and the collection time in one channel was $250 \mathrm{~ms}$. Typically, 5-10 scans were added to acquire a single spectrum. Energy referencing was not applied, spectra are represented as acquired. Powder-like samples were evenly laid on one side of a double-sized adhesive tape, the other side being attached to the sample holder of the XPS instrument. The samples were evacuated at room temperature, and then inserted into the analysis chamber of the XPS instrument. No other treatments were applied.

\subsubsection{Dynamic mechanical analysis (DMA)}

Mechanical properties of samples were determined by dynamic mechanical analysis using a TA Instruments DMA Q800 instrument. Stiffness vs. temperature curves were obtained by fixing samples in single cantilever clamps. Tests were performed at heating rate of $5 \mathrm{~K} / \mathrm{min}$ and using a frequency of $18 \mathrm{~Hz}$. The frequency was chosen according to a preliminary frequency sweep measurement of the sample, which showed the most linear response in this region. PTFE and aluminum sheets with comparable size were also measured as reference materials.

\subsubsection{Catalytic performance tests}

Catalytic performance tests were conducted in a U-shaped continuous flow glass reactor. In a typical experiment $0.01 \mathrm{~g}$ of catalyst was mechanically dispersed in $1 \mathrm{~g}$ of Stöber silica in an agate mortar, where the silica was used as catalyst bed. Cyclohexene was introduced using a temperature controlled saturator, where the gas was forced through short tube shaped glass inserts to prevent small droplet from escaping the saturator. Gas flows 
were set by using mass flow controllers. Gas products were analyzed by injecting a fixed amount of gas $(250 \mu \mathrm{l})$ into an Agilent GC 6820 gas chromatograph equipped with a flame ionization detector. Peak areas were integrated using the Cerity factory provided software.

TOF was calculated using metal concentration data obtained with EDS and surface atom numbers calculated from average particle diameters scaled on TEM micrographs. All particles were assumed to be spherical for the calculation of surface atoms. For the determination of TOF, data recorded after 10 minutes of reaction were used in all cases. 


\section{Results and discussion}

\subsection{Preparation and characterization of mesoporous amorphous carbon supported Pd catalysts with tunable specific surface area and pore size}

\subsubsection{Preparation of mesoporous carbon supports}

Mesoporous amorphous carbon materials were prepared by the chemical vapor infiltration method (CVI). Ludox ${ }^{\circledR}$ silica colloids with different particle size were chosen as template materials. The average size of SM30, AS40 and HS40 silica particles were found to be 5-8, 13-20 and 8-11 nm, respectively. Generally, $30 \mathrm{~g}$ of silica template was used for the synthesis, where both single and bicomponent mixtures of 1:3, 1:1 and 3:1 ratios were applied, forming 12 different compositions in all. The solvents were evaporated in a rotary evaporator at 363-423 K, which took 1-1.5 h. The solid matter was further dried in an oven at $423 \mathrm{~K}$ in air overnight to remove the remaining moisture. The resulting template material was then placed in a quartz boat, which was then slid into a quartz tube. The tube was purged with $300 \mathrm{~cm}^{3} / \mathrm{min}$ nitrogen flow for 10 min prior to starting the reaction. A schematic of this arrangement is depicted in Fig. 5.1. The tube was then pushed into a preheated (973 K) tube furnace and acetylene with a flow rate of $30 \mathrm{~cm}^{3} / \mathrm{min}$ was introduced to start the reaction. After $60 \mathrm{~min}$ of reaction time the acetylene flow was stopped and the reactor temperature was raised to $1173 \mathrm{~K}$ in $8 \mathrm{~min}$. The carbonization procedure was continued for $60 \mathrm{~min}$.

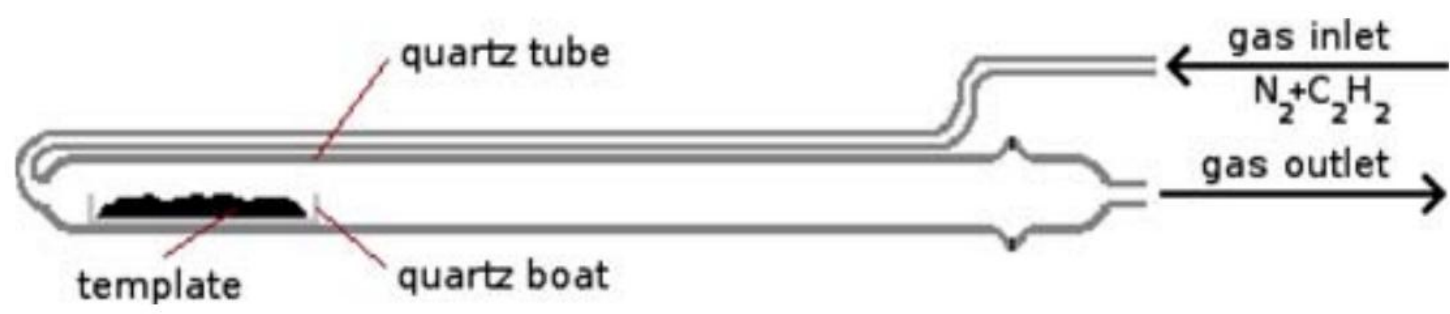

Figure 5.1. Schematic of reactor arrangement for the preparation of mesoporous amorphous carbon structures

After reaction time the quartz reactor was removed from the furnace and was allowed to cool to room temperature. The silica template was removed by etching with $150 \mathrm{~cm}^{3} 40 \%$ HF solution for $12 \mathrm{~h}$. Samples were washed with distilled water several times then dried overnight at $353 \mathrm{~K}$ in air. This procedure was repeated once more to ensure all silica template was removed. A simplified workflow of the synthesis is depicted in Fig. 5.2. 


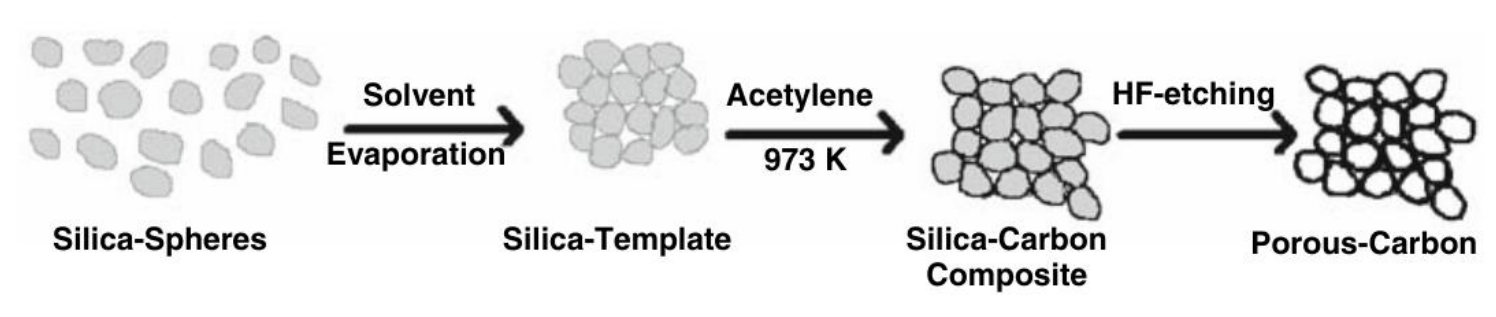

Figure 5.2. Scheme of synthesis procedure of amorphous carbon support

\subsubsection{Characterization of silica templates and mesoporous carbon supports}

(a)

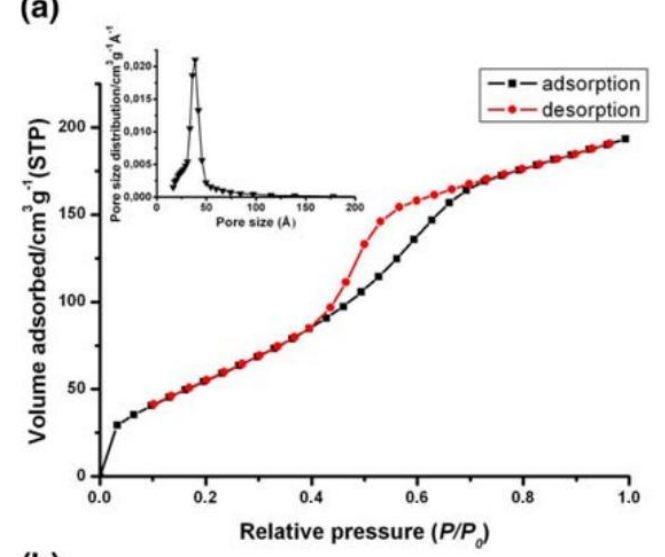

(b)

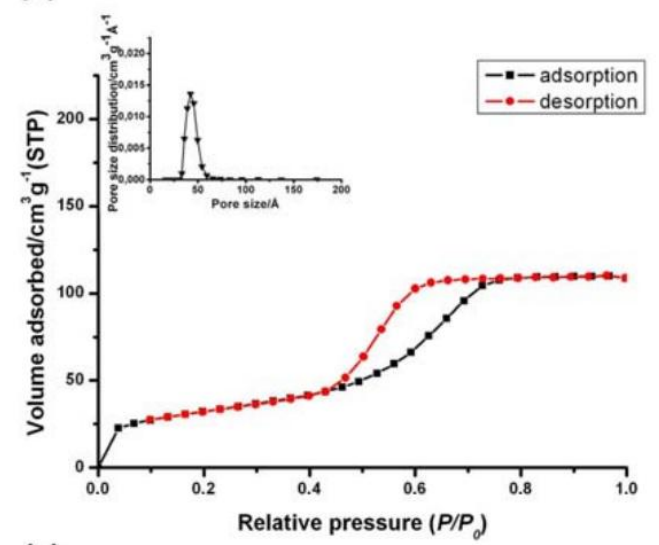

(c)

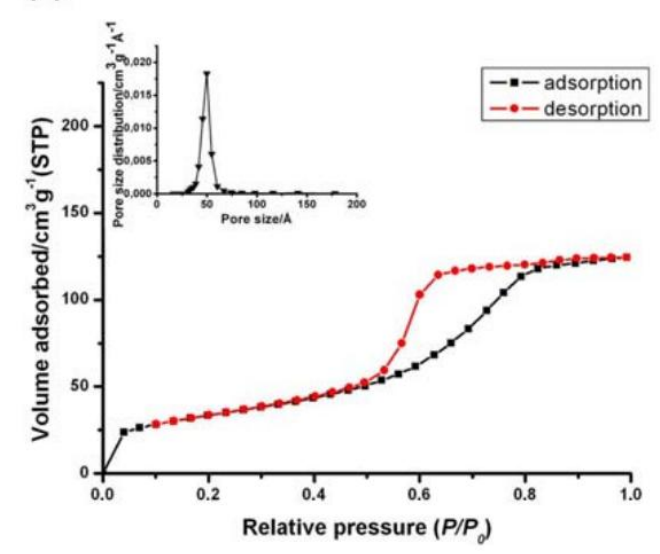

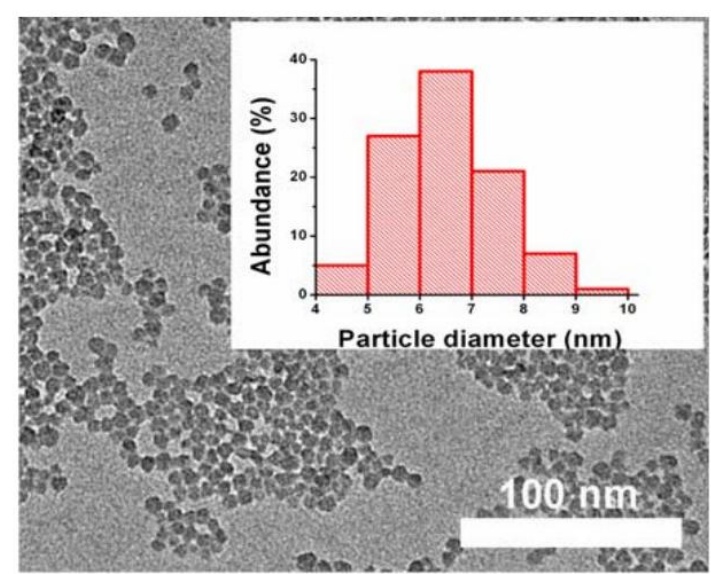
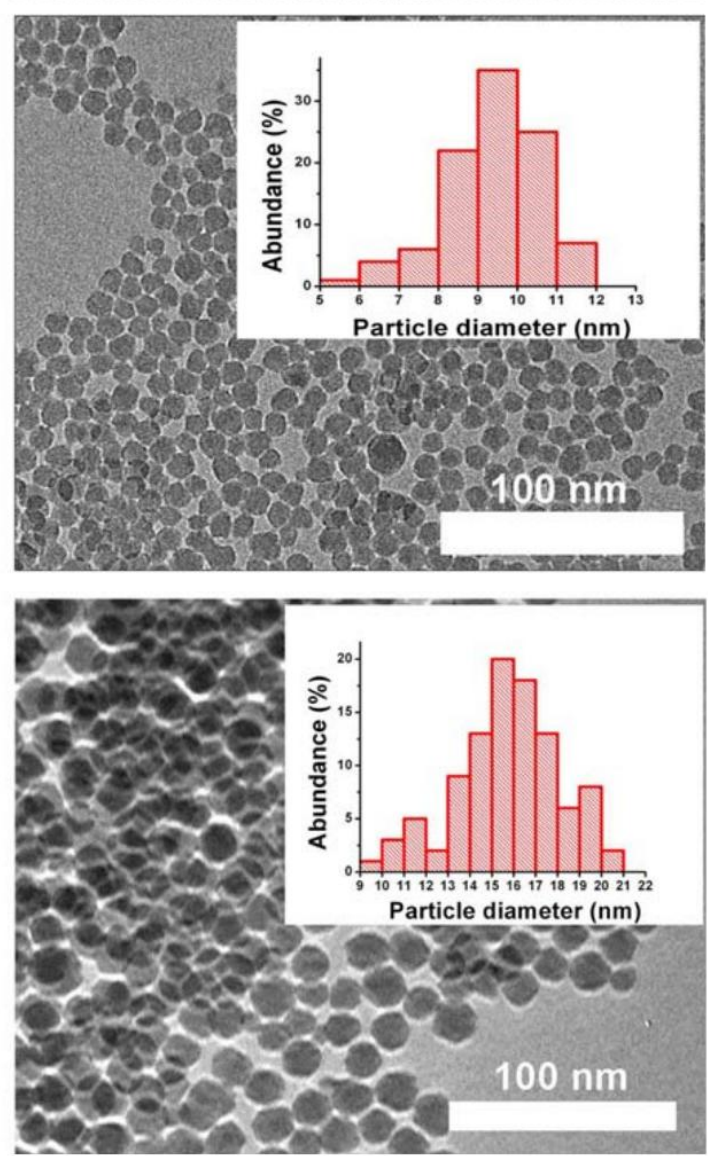

Figure 5.3 Nitrogen adsorption isotherms, pore size distributions (as insert in isotherms), TEM images and particle size distribution histograms (as insert in TEM micrographs) of template materials a) SM30, b) HS40 and c) AS40 
As first step the dried Ludox ${ }^{\circledR}$ template materials were investigated, where the silica spheres form a mesoporous structure consisting of few mm aggregates. Both TEM and nitrogen adsorption-desorption measurements were conducted to determine particle size and pore size distribution of samples. At least 200 particles were scaled manually on TEM micrographs. Adsorption isotherms, pore size distributions, TEM micrographs and histograms of SM30, HS40 and AS40 non-mixed templates are presented in Fig. 5.3.

Table 5.1. Specific surface area, specific pore volume, average pore diameter and average particle diameter of all silica template materials used.

\begin{tabular}{lcccc}
\hline Template & $\mathrm{S}_{\mathrm{BET}}\left(\mathrm{m}^{2} / \mathrm{g}\right)$ & $\mathrm{V}_{\text {pore }}\left(\mathrm{cm}^{3} / \mathrm{g}\right)$ & $\mathrm{d}_{\text {av.pore }}(\mathrm{nm})$ & $\mathrm{d}_{\text {av.particle }}(\mathrm{nm})$ \\
\hline SM30 & 239 & 0.35 & 4 & $5-8$ \\
HS40 & 112 & 0.19 & 4.7 & $8-11$ \\
AS40 & 118 & 0.2 & 5 & $14-18$ \\
\hline
\end{tabular}

Nitrogen sorption isotherms of all silica templates presented in Fig 5.3. are characteristic of Type IV as defined by IUPAC. The SM30 dry silica material exhibited a specific surface area value of $239 \mathrm{~m}^{2} / \mathrm{g}$, average pore size of $4 \mathrm{~nm}$, specific pore volume of $0.35 \mathrm{~cm}^{3} / \mathrm{g}$ and an average particle diameter of $5-8 \mathrm{~nm}$. The HS40 dried material showed a specific surface area value of $112 \mathrm{~m}^{2} / \mathrm{g}$, average pore size of $4.7 \mathrm{~nm}$, specific pore volume of $0.19 \mathrm{~cm}^{3} / \mathrm{g}$ and an average particle diameter of $8-11 \mathrm{~nm}$. The AS40 dry template showed specific surface area value of $118 \mathrm{~m}^{2} / \mathrm{g}$, average pore size of $5 \mathrm{~nm}$, specific pore volume of $0.2 \mathrm{~cm}^{3} / \mathrm{g}$ and an average particle diameter of $14-18 \mathrm{~nm}$. This data is also tabulated in Table 5.1. To follow the changes in pore system characteristics happening during the synthesis procedure, nitrogen sorption isotherms were acquired at each step of the synthesis. The resulting isotherms, $S_{\mathrm{BET}}$ and $\mathrm{V}_{\text {pore }}$ values are presented in Fig. 5.4. 

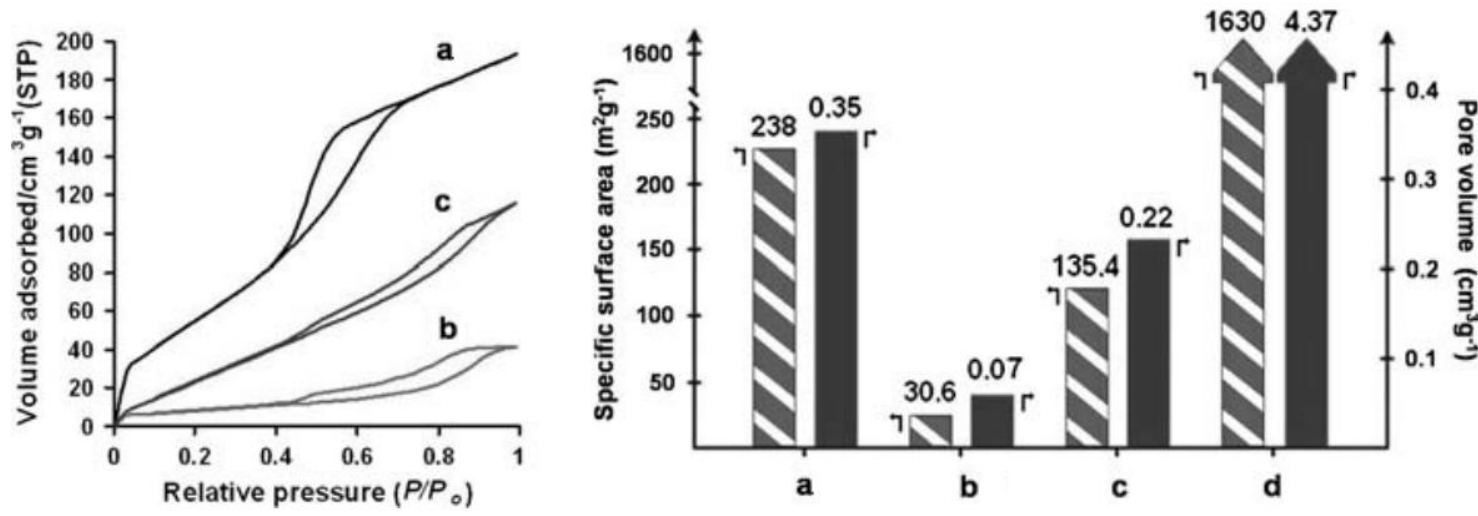

Figure 5.4. Pore system analysis of materials during each synthesis step performed on SM30 silica template. a) dried SM30 template, b) SM30 template after synthesis (silica-carbon composite), c) SM30 silica-carbon composite after grinding in mortar, $d$ ) resulting mesoporous carbon

Sorption isotherms of silica template, silica-carbon composite and ground silica-carbon composite are presented in Fig 5.4. All isotherms exhibit characteristics of Type IV isotherms defined by IUPAC. As it is clearly seen on the isotherms presented in Fig 5.4., the pore structure of the template material changes drastically after reaction, as the initial $\mathrm{S}_{\mathrm{BET}}$ and $\mathrm{V}_{\text {pore }}$ values of $238 \mathrm{~m}^{2} / \mathrm{g}$ and $0.35 \mathrm{~cm}^{3} / \mathrm{g}$, respectively drop to $31 \mathrm{~m}^{2} / \mathrm{g}$ and $0.07 \mathrm{~cm}^{3} / \mathrm{g}$, respectively representing an $80 \%$ drop. This means that during synthesis the forming amorphous carbon layer partially at least blocks the few nanometer pores found between the silica spheres of the silica template. This blockage of pores most likely occurs at the entrance of the pores. To prove this, some of the material was ground in a mortar then investigated again by nitrogen sorption. This resulted in a rise of $S_{\text {BET }}$ and $V_{\text {pore }}$ values to $135 \mathrm{~m}^{2} / \mathrm{g}$ and $0.22 \mathrm{~cm}^{3} / \mathrm{g}$, respectively, which proves the above presented theory. It is noteworthy, that during the grinding of $1 \mathrm{~mm}$ silica-carbon composite particles no white areas corresponding to pure silica were found in the resulting smaller pieces, all areas were found to be evenly black. This proves that during synthesis the carbon vapor can infiltrate even into the biggest silica aggregates of the template, thus the carbon coverage on all nanoparticles is even.

As discussed in the literature section, the $\mathrm{S}_{\mathrm{BET}}$ and $\mathrm{V}_{\text {pore }}$ values were controlled by changing the precursor/silica ratio [21], which has a disadvantage of producing non-uniform sidewall thicknesses. To provide a means to fine-tune the specific surface area and total pore volume of the resulting carbon structure without such inconsistencies, binary mixtures with weight ratios of 1:3, 1:1, 3:1 have also been prepared from the previously mentioned templates exhibiting various particle size. This method both ensured standard growth procedure and a good dispersion of particles. The resulting $S_{\mathrm{BET}}$ and $\mathrm{V}_{\text {pore }}$ values of all as prepared samples are tabulated in Table 5.2. 
Table 5.2. Specific surface area and total pore volume of mesoporous carbon materials prepared from various silica template mixtures. It is well seen that by selecting the right template mixture the $S_{\mathrm{BET}}$ and $V_{\text {pore }}$ of the resulting material can be well tuned in ranges starting from $\sim 620 . \mathrm{m}^{2} / \mathrm{g}$ to $\sim 1600 \mathrm{~m}^{2} / \mathrm{g}$ and $\sim 1$ $\mathrm{cm}^{3} / \mathrm{g}$ to $\sim 4.4 \mathrm{~cm}^{3} / \mathrm{g}$, respectively.

Used template (weight ratio) $\quad \mathrm{S}_{\mathrm{BET}}\left(\mathrm{m}^{2} / \mathrm{g}\right) \quad \mathrm{V}_{\text {pore }}\left(\mathrm{cm}^{3} / \mathrm{g}\right)$

\begin{tabular}{lcl}
\hline AS40 & 624 & 0.99 \\
AS40:HS40 = 3:1 & 755 & 2.49 \\
AS40:HS40 = 1:1 & 745 & 2.88 \\
AS40:HS40 = 1:3 & 854 & 3.17 \\
HS40 & 1017 & 3.55 \\
SM30:HS40 = 3:1 & 1018 & 3.45 \\
SM30:HS40 = 1:1 & 1300 & 3.85 \\
SM30:HS40 = 1:3 & 1421 & 4.12 \\
SM30 & 1630 & 4.37 \\
SM30:AS40 = 3:1 & 1439 & 3.72 \\
SM30:AS40 = 1:1 & 961 & 2.64 \\
SM30:AS40 = 1:3 & 868 & 2.23 \\
AS40 & 624 & 0.99 \\
\hline
\end{tabular}

A clear tendency is visible in $S_{B E T}$ and $V_{\text {pore }}$ with the changing of the mixture composition at all prepared series. While the amorphous carbon synthesized using AS40 template exhibited $\mathrm{S}_{\mathrm{BET}}$ and $\mathrm{V}_{\text {pore }}$ values of $624 \mathrm{~m}^{2} / \mathrm{g}$ and $0.99 \mathrm{~cm}^{3} / \mathrm{g}$, respectively, samples with added SM30 ratios of 1:3, 1:1 and $3: 1$ showed $868 \mathrm{~m}^{2} / \mathrm{g}, 2.23 \mathrm{~cm}^{3} / \mathrm{g}, 961 \mathrm{~m}^{2} / \mathrm{g}, 2.64$ $\mathrm{cm}^{3} / \mathrm{g}$ and $1439 \mathrm{~m}^{2} / \mathrm{g}, 3.72 \mathrm{~cm}^{3} / \mathrm{g}$, respectively. The carbon material synthesized using pure SM30 had the highest values of $1630 \mathrm{~m}^{2} / \mathrm{g}$ and $4.37 \mathrm{~cm}^{3} / \mathrm{g}$. It is clearly seen, that with the growth in ratio of the silica template component consisting of smaller particles affects specific surface area and specific pore volume positively. Other sample series exhibited similar tendencies.

SM30 derived AC supports were chosen for later studies with noble metal modification, as this material exhibited the most advantageous properties. To determine the thermal stability of these materials thermogravimetric measurements were performed. Samples were inspected in both oxidative and inert atmospheres at $10^{\circ} \mathrm{C} / \mathrm{min}$ heating rate. TG curves are presented in Fig. 5.5. A less than $3 \mathrm{wt} \%$ of weight loss was detected for AC sample annealed in inert atmosphere, however in air a significant weight loss started at $773 \mathrm{~K}$ which ended around $1123 \mathrm{~K}$. These results are in the same regime as most other common carbon 
allotropes, which clearly demonstrate the thermal stability and suitability of the material as catalyst support.

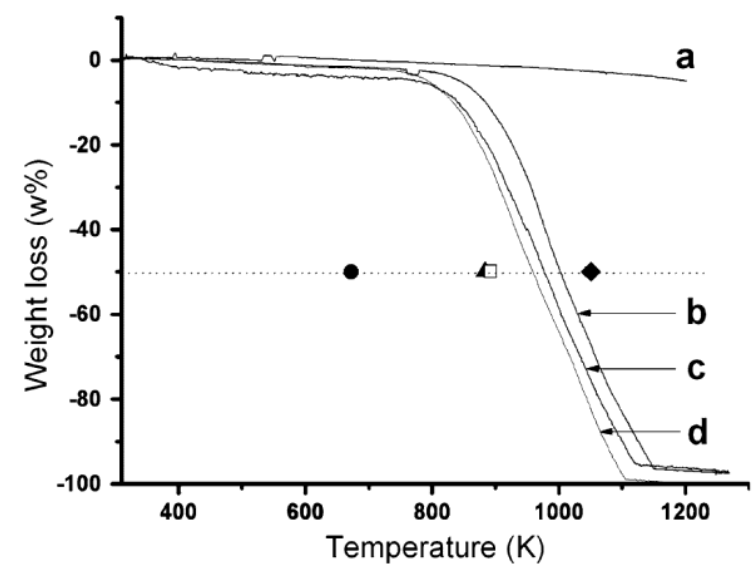

Figure 5.5. Thermogravimetric curves of SM30 derived AC analyzed in a) nitrogen and b) oxidative atmosphere, c) commercial activated carbon (Fluka) in air and d) MWCNT (made in our lab) in air. Literature data at $\mathbf{5 0 \%}$ weight loss is also presented for comparison: $\bullet$ amorphous carbon, $\boldsymbol{\Delta}$ pure $\mathbf{C 6 0}$, $\bullet$ diamond, $\square$ MWNCT graphitized at $3000 \mathrm{~K}$.

\subsubsection{Modification of mesoporous amorphous carbon structures with palladium nanoparticles}

To produce Pd modified mesoporous carbon (MC) structures, similar synthetic routes were taken for the preparation of the carbon support as described in section 5.1.1. In order to study the effect of synthesis temperature on nanoparticles and later catalytic conversion, samples with carbonization temperature of $973 \mathrm{~K}$ have also been created besides the original temperature of $1173 \mathrm{~K}$. For easy referencing, these samples were denoted as MC77 and MC79, respectively, which stand for Mesoporous Carbon $700+700^{\circ} \mathrm{C}$ or Mesoporous Carbon $700+900^{\circ} \mathrm{C}$.

Pd nanoparticles were introduced by selecting two different methods. The first method is the well-established wet impregnation procedure, where $22 \mathrm{mg}$ of Pd-acetate was dissolved in $100 \mathrm{ml}$ of toluene, and then $200 \mathrm{mg}$ of carbon material was added to the solution. The mixture was ultrasonically treated in an ultrasonic bath for $15 \mathrm{~min}$, and then stirred with a magnetic stirrer for $24 \mathrm{~h}$ at room temperature. The solvent was finally separated with centrifugation, and the carbon material was dried overnight at $353 \mathrm{~K}$. Nanoparticles were formed by thermal annealing of the samples in Ar atmosphere, where the samples were placed in a quartz tube using ceramic crucibles. The tube was purged with pure argon (300 $\mathrm{ml} / \mathrm{min}$ ) for $10 \mathrm{~min}$ to remove oxygen, after which the tube was placed into a preheated furnace. The furnace was operated at $458 \mathrm{~K}$ for $2 \mathrm{~h}$, then was further heated to $653 \mathrm{~K}$ at a rate of $20 \mathrm{~K} / \mathrm{min}$ and was left there for $1 \mathrm{~h}$. The tube was finally allowed to cool naturally to room 
temperature outside of the oven. Samples modified with this method have got an "-I" suffix in their names (MC77-I and MC79-I).

The second method, which is from now on called the "Pd-sol method", required a separate synthesis of Pd nanoparticles and a slight modification of the original synthesis method of the mesoporous carbon support. Pd nanoparticles were synthesized as described by Nutt et al [280]. The as-prepared Pd-sol was simply added to the SM30 colloid silica sol prior to vacuum evaporation, which ensured a very even distribution of nanoparticles. The mixture was then treated as described earlier to obtain the carbon deposition on the sample; no further modifications were made to the procedure. Samples prepared with this method have got a "-C" suffix in their names (MC77-C and MC79-C).

For the removal of silica templates a second method with $\mathrm{NaOH}$ etching was introduced besides the previously described HF method. Shortly, the $\mathrm{NaOH}$ etching was performed in a PTFE coated steel autoclave $\left(400 \mathrm{~cm}^{3}\right.$ total volume), where the composite material was mixed with $10 \mathrm{~mol} / \mathrm{dm}^{3} \mathrm{NaOH}$ solution). The sealed autoclave was then placed in a furnace preheated to $413 \mathrm{~K}$ for $24 \mathrm{~h}$. Afterwards, the material was cleaned with filtration and was washed with distilled water until neutral $\mathrm{pH}$ was reached. The samples were finally dried in air at $353 \mathrm{~K}$ overnight. This method was repeated to ensure all silica material was removed. For easier understanding of the procedures taken to synthesize Pd modified mesoporous carbon, a schematic is provided in Fig. 5.6.

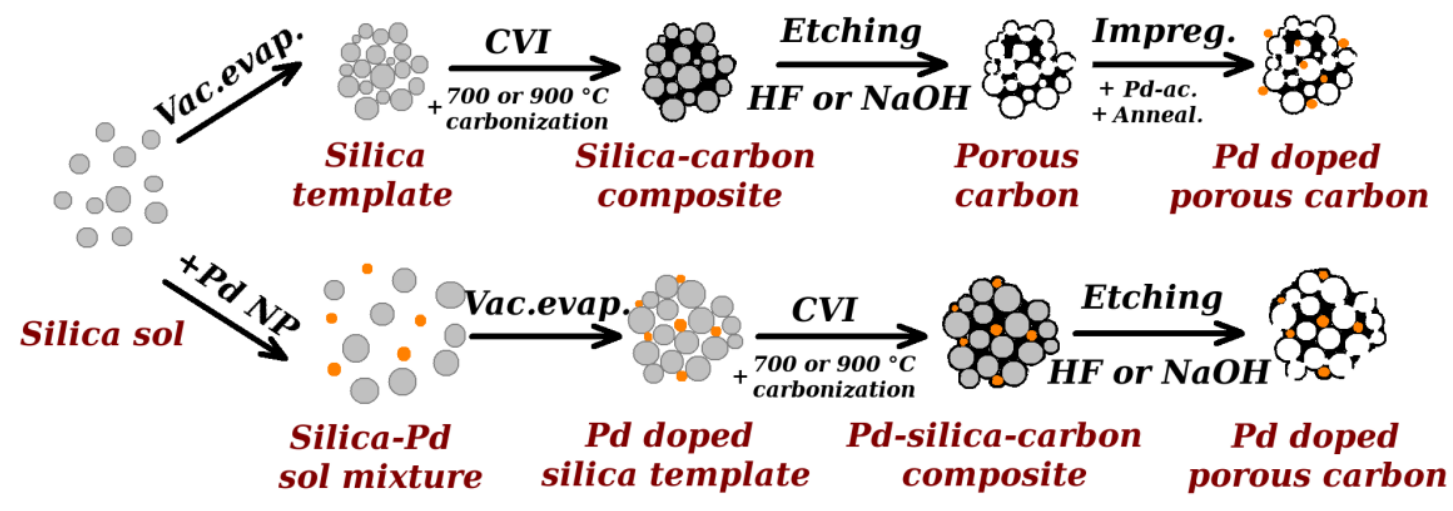

Figure 5.6. A simplified schematic of the different routes taken for the synthesis of Pd/mesoporous carbon catalysts. The upper path represents a catalyst prepared with the wet impregnation method, while the bottom path shows the "Pd-sol method".

\subsubsection{Characterization of mesoporous carbon supported palladium catalysts}

\subsection{4.a. Nitrogen adsorption measurements}



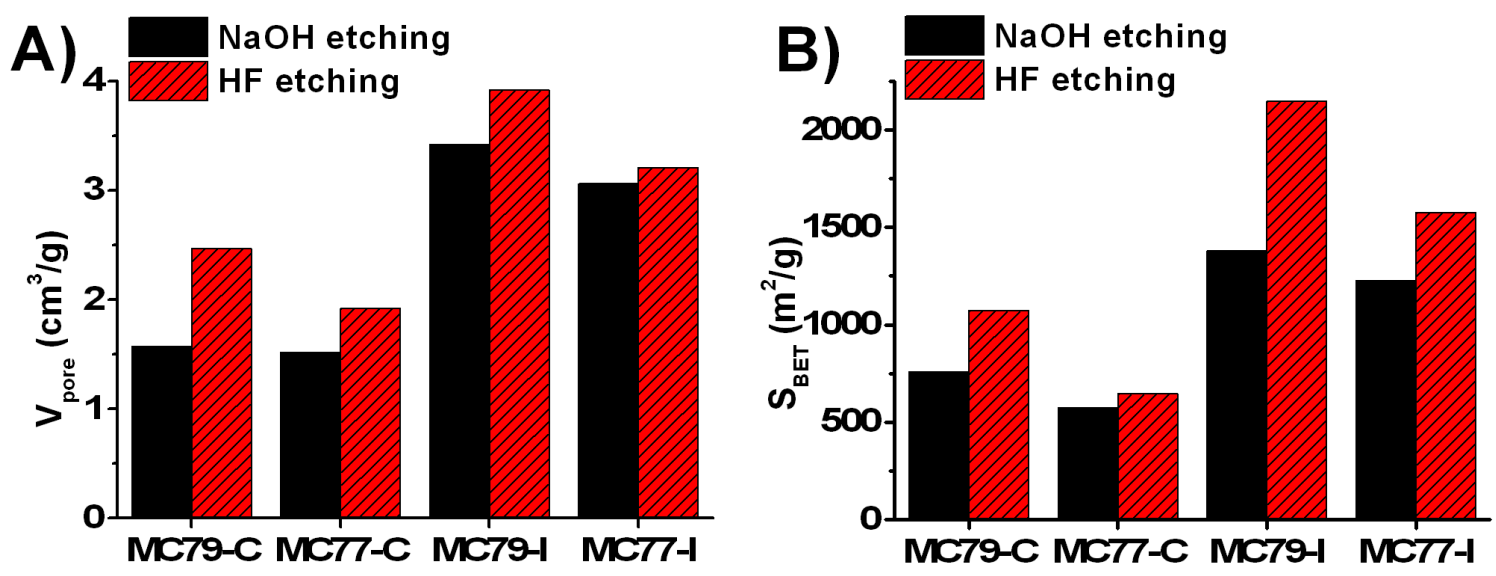

Fig. 5.7. A) Total pore volume and B) specific surface area of the various Pd/mesoporous carbon catalysts. Samples prepared with the "Pd-sol method" clearly demonstrate about twice as low total pore volume and specific surface area then samples prepared with the wet impregnation method. HF etching mostly resulted in materials with higher total pore volume and specific surface area compared to the $\mathrm{NaOH}$ treated catalysts.

Nitrogen adsorption measurements were conducted on both the raw mesoporous carbon supports carbonized at $973 \mathrm{~K}$ and $1173 \mathrm{~K}$ and the mesoporous carbon supported $\mathrm{Pd}$ nanoparticle catalysts. The newly created raw materials MC77 and MC79 exhibited outstanding $\mathrm{S}_{\mathrm{BET}}$ values of $1695 \mathrm{~m}^{2} / \mathrm{g}$ and $1746 \mathrm{~m}^{2} / \mathrm{g}$, respectively, which is considerably higher than most commercially available carbon materials, which are only in the range of a few hundred $\mathrm{m}^{2} / \mathrm{g}$ or less (Fig. 5.8.). The total pore volume of samples was equally found to be above average with values of $3.15 \mathrm{~cm}^{3} / \mathrm{g}$ and $3.51 \mathrm{~cm}^{3} / \mathrm{g}$ for samples MC77 and MC79, respectively. The introduction of $\mathrm{Pd}$ into the carbon structure had limited effect on $\mathrm{V}_{\text {pore }}$ and $\mathrm{S}_{\mathrm{BET}}$ values, depending on the method used. As it is clearly seen on Fig 5.7.B samples prepared with the "Pd-sol method" (MC77-C and MC79-C) showed S $\mathrm{BET}_{\text {values 570-1070 }}$ $\mathrm{m}^{2} / \mathrm{g}$ which are to $70 \%$ to $40 \%$ smaller than values of raw samples. Total pore volumes showed equally high drops. This is most probably caused by the presence of sodium citrate and tannic acid used for the preparation of the Pd sol, which function as excess carbon source during synthesis, as after vacuum evaporation of the silica template the nanoparticles are evenly covered by these organic substances. By taking into account the excess carbon source and the different decomposition characteristics of these substances, a different resulting carbon structure is not unlikely. This assumption is validated by the visible difference in carbon structures between catalysts prepared with the "Pd-sol" and wet impregnation methods on TEM micrographs as discussed later (Fig 5.9). Samples prepared with the wet impregnation method showed $S_{B E T}$ and $V_{\text {pore }}$ values of $1230 \mathrm{~m}^{2} / \mathrm{g}$ to $2150 \mathrm{~m}^{2} / \mathrm{g}$ and 3.06 $\mathrm{cm}^{3} / \mathrm{g}$ to $3.92 \mathrm{~cm}^{3} / \mathrm{g}$, respectively, which are in the range of the original raw materials. 
A)

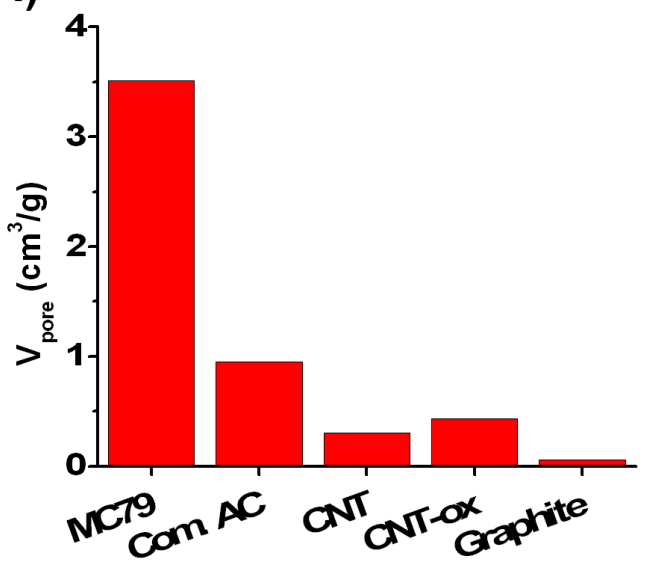

B)

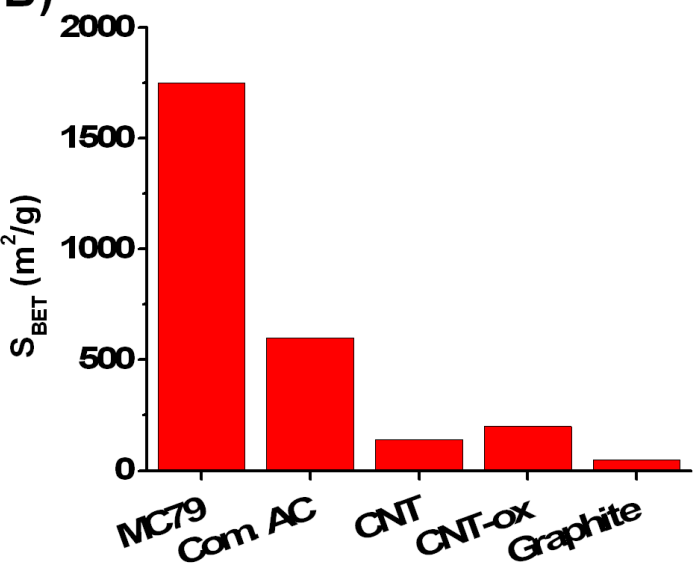

Figure 5.8. A) Total pore volume and B) specific surface area of mesoporous carbon carbonized at 1173

$\mathrm{K}$ has outstanding specific surface area compared to other carbon allotropes (commercial activated carbon (Sigma-Aldrich) - Com. AC, carbon nanotubes - CNT, oxidized carbon nanotubes - CNT-ox and graphite)

Interestingly, the method used for etching the template seems to affect the specific surface area and total pore volume. Samples etched with $\mathrm{NaOH}$ show constantly lower $\mathrm{S}_{\mathrm{BET}}$ and moderately lower " $\mathrm{V}_{\text {pore }}$ " values then their HF etched counterparts, which could mean that HF seems to be a more efficient agent for the removal of the template. This may be due to the fact that while the reaction of $\mathrm{SiO}_{2}$ and $\mathrm{HF}$ produces $\mathrm{SiF}_{4}$, which leaves the system in a gaseous form, the reaction of $\mathrm{NaOH}$ and $\mathrm{SiO}_{2}$ on the other hand forms waterglass, which is a liquid often with high viscosity.

Carbonization temperature also affected $\mathrm{S}_{\mathrm{BET}}$, where a higher carbonization temperature resulted in a higher specific surface area for all samples.

\subsection{4.b. Transmission electron microscopy}

TEM micrographs were acquired for all Pd modified samples (Fig. 5.9.). The images clearly show the amorphous nature of the carbon structure of all samples, however clear differences are discernable between samples modified with the "Pd-sol" and wet impregnation methods. Samples prepared with the wet impregnation method (MC77-I and MC79-I) clearly showed a foamy structure with relatively spacious pores and thin walls, but samples prepared with the "Pd-sol" method (MC77-C and MC79-C) exhibited a more dense structure. These findings are in agreement with observations made at nitrogen sorption, as discussed earlier. Nitrogen sorption data also indicated a difference between etching methods implemented, but this difference is not evident on the general look of the carbon structures. This could mean, that the etching agent does not have any effect on the carbon structure, only on the efficiency of the template removal. 



Figure 5.9. TEM micrographs of Pd-MC nanocomposites. I: MC79-C, II: MC77-C, III: MC79-I, IV: MC77-I. Micrographs with captions " $A$ " and " $B$ " represent etching performed with $\mathrm{NaOH}$ and $\mathrm{HF}$ respectively. Samples prepared with the "Pd-sol method" clearly demonstrate a more dense structure, hence the twice lower specific surface area if compared to samples prepared with the wet impregantion method.

Pd nanoparticles are well dispersed within the carbon structure, no particles were found outside the carbon matrix. Particles supported on impregnated samples MC79-I and MC77-I exhibited diameters of 3.4-3.6 $\mathrm{nm}$ and 3.0-4.2 $\mathrm{nm}$, respectively, however particles on carbon structures modified by the "Pd-sol method" showed higher values of 8.0-8.9 nm and 4.2-5.6 $\mathrm{nm}$ for MC79-C and MC77-C, respectively (Fig. 5.10.). The average diameter of the particles in the original sol was $4.9 \mathrm{~nm}$, thus the increased diameters found in samples carbonized at $1173 \mathrm{~K}$ is assumed to be the result of sintering. Interestingly, the diameters found for samples carbonized at $973 \mathrm{~K}$ (MC77-C) do not show significantly higher values then found for the original sol, which means, that particles supported on MC are mostly resistant to sintering up 
to $973 \mathrm{~K}$. Such clear difference in diameters caused by carbonization temperatures is not evident on samples prepared with the wet impregnation method. Particles exhibit nearly identical diameters on samples carbonized at $1173 \mathrm{~K}$, regardless of the etching method used. Particles found on sample MC77-I etched with $\mathrm{NaOH}$ show essentially identical diameter if compared to MC79-I samples, however particles found on NaOH etched MC77-I are slightly larger in diameter. This could mean that carbon structures carbonized at higher temperatures result in more stable structures for Pd nanoparticles.

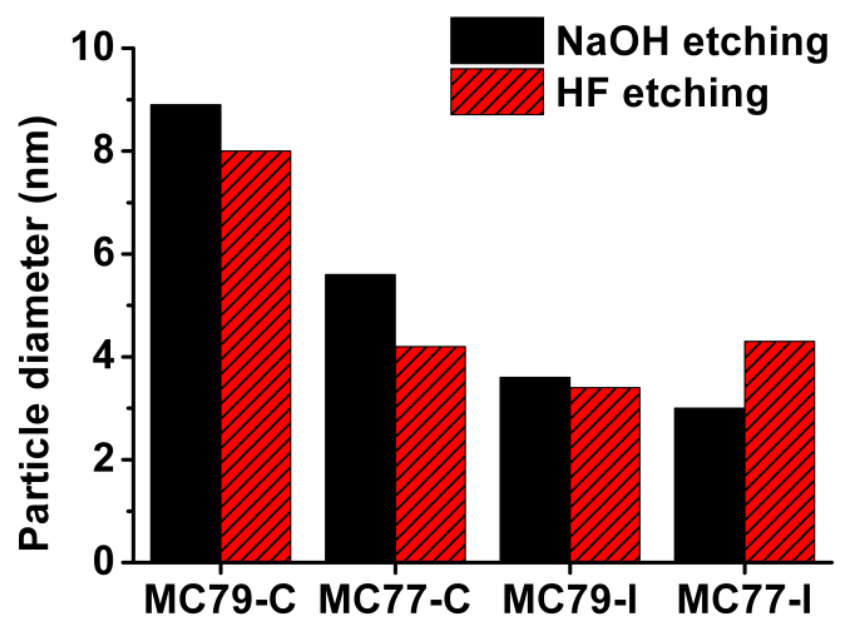

Fig. 5.10. Average diameter of Pd nanoparticles supported on various mesoporous carbon supports. Samples made by impregnation resulted in 3.4-4.2 $\mathrm{nm}$ Pd nanoparticles, however premixing of the assynthesized Pd nanoparticles with the silica template (Pd-sol method) resulted in larger particle diameter (4.2-8.9 nm) due to the sintering of the particles at higher carbonization temperature. Etching method has no significant effect on the particle diameter.

\subsection{4.c. X-ray photoelectron spectroscopy}

All four mesoporous carbon supported Pd nanoparticle catalysts (MC79-C, MC77-C, MC79-I, MC77-I) were investigated by XPS. Low resolution survey, and high resolution O 1s, C 1s, Na 1s and F 1s (where relevant) spectra were taken. Since the spectra obtained were practically energy correct, no further calibration was applied. The $\mathrm{O} 1 \mathrm{~s}$ signal can be fitted with three components in all cases. The most intense component is at $534.3 \mathrm{eV}$, supposedly from water, but in $\mathrm{NaOH}$ treated samples the most intense signal is at $532.5 \mathrm{eV}$, due to $\mathrm{OH}$ groups.

Very intense strongly asymmetric C 1s spectra were recorded in all four Pd-loaded samples. The peak maxima were at $285.1 \mathrm{eV}$, characteristic of amorphous carbon. At least three, or even more components could be identified on the high binding energy side of the spectra. These all originate from various carbon-oxygen groups, among them from - $\mathrm{COOH}$. 
Weak signal maxima between 1072.3-1072.6 eV can be attributed to sodium in $\mathrm{NaOH}$ etched samples. The high binding energy reveals that sodium is very positive, possibly in the form of oxide, hydroxide, or halogenide. Fluorine 1s spectra were also taken in relevant samples. This very low intensity signal peaks at $689.7 \mathrm{eV}$, marking a very positive fluorine ion. This binding energy is much higher than found in alkali-fluorides, but matches the values found in C-F containing compounds.

\subsection{4.d. Catalytic tests}

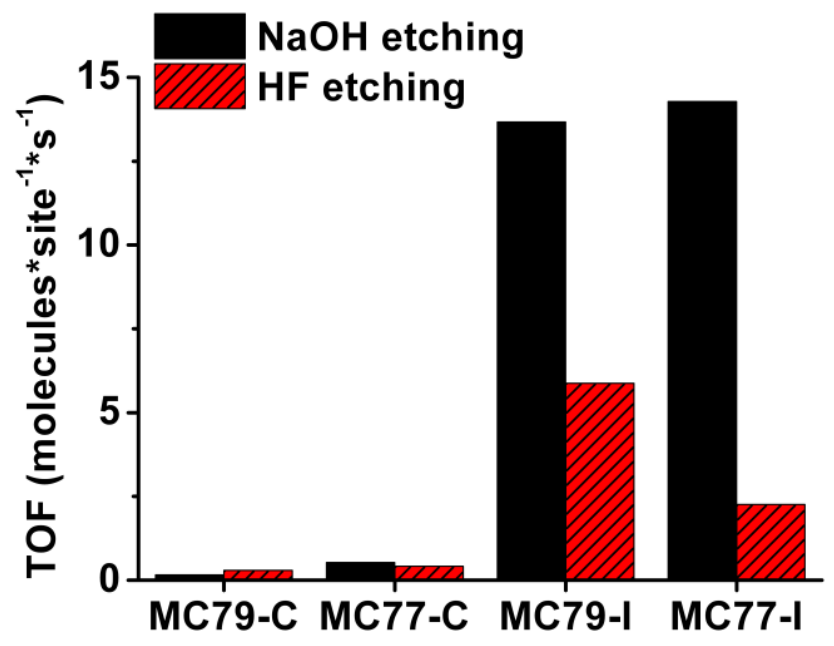

Fig. 5.11. Catalytic performance of various mesoporous carbon supported Pd catalysts in cyclohexene hydrogenation to cyclohexane at $313 \mathrm{~K}$. Catalysts with nanoparticles impregnated onto $\mathrm{NaOH}$ etched mesoporous carbon show extremely high TOF values if compared to HF etched ones (MC79-I and MC77I). Samples prepared with the "Pd-sol method" (MC79-C and MC77-C) show very low activity regardless of synthesis temperature, which is a result of nanoparticles trapped into the pores of the support during the carbonization process of the mesoporous carbon synthesis.

Catalytic hydrogenation of cyclohexene to cyclohexane was chosen as model reaction for the test of catalysts. A great difference in TOF is visible between samples prepared with the "Pd-sol" and wet impregnation methods. MC79-C and MC77-C samples exhibited TOF values of 0.5 molecules $\cdot \operatorname{sit}^{-1} \cdot \mathrm{s}^{-1}$ or smaller, regardless of carbonization temperature or etching method used. Samples prepared with the wet impregnation method however, showed considerably higher TOF of 5.9-13.7 and 2.2-14.3 molecules $\cdot \operatorname{site}^{-1} \cdot \mathrm{s}^{-1}$ for MC79-I and MC77I, respectively. The low TOF values of samples prepared with the "Pd-sol method" could be mostly attributed to the encapsulation of nanoparticles into the carbon matrix during the synthesis process, as the extra organics used as stabilizing agent for the Pd sol possibly form a more dense carbon structure. This assumption is proved by both TEM and BET results, as 
described earlier. Another possible effect contributing to low TOF values could be the enlarged particle size due to sintering that occurs at elevated temperatures. This effect however can be considered to be much smaller however, as HF etched samples prepared with the wet impregnation and "Pd-sol methods" carbonized at 973K exhibited virtually identical particles diameters, yet the TOF values differ greatly.

Unexpectedly high TOF values of $\sim 14$ molecules $\cdot \operatorname{site}^{-1} \cdot \mathrm{s}^{-1}$ were obtained for impregnated samples etched with $\mathrm{NaOH}$, which are several times higher than TOF values found in other publications. It must be noted that these extreme values are found for samples exhibiting 25-35\% lower specific surface area if compared to samples etched with HF. These seemingly controversial results could be attributed to the promoting effect of the minute amounts of sodium present in the carbon structure remaining from the etching process with $\mathrm{NaOH}$. An elevated concentration of hydroxyl groups in these samples could also contribute to this extraordinary activity. The presence of both $\mathrm{Na}$ and hydroxyl groups are confirmed by XPS data. Interestingly, the fluorine containing groups found in HF etched samples did not seem to have any major effect on the catalytic activity of these catalysts.

\subsection{Preparation and characterization of palladium and palladium-oxide nanoparticle modified oxidized carbon nanotubes.}

A systematic oxidation of carbon nanotubes with nitric acid has been performed in order to reveal not well comprehended aspects of functionalization, including effects on specific surface area, nanotube structure and on nanoparticle modified samples, supported nanoparticle size and/or catalytic activity. For this purpose several different sample series were synthesized using a wide range of oxidation times ranging from $0 \mathrm{~h}$ to $24 \mathrm{~h}$. Nanotubes were modified with both Pd and PdO nanoparticles in order to reveal possible differences in effects of functionalization on oxide or metallic nanoparticle size. To be able to separately study the effects of residual synthetic (post-CCVD) impurities, functional groups, oxidation debris and structural defects, a Soxhlet-extractor enhanced acetone washing process was also incorporated into the overall synthesis procedure.

\subsubsection{Nitric acid assisted carbon nanotube oxidation and modification with palladium and palladium-oxide nanoparticles}

MWCNTs prepared by the catalytic chemical vapor deposition (CCVD) method in our lab were used as basis material for all experiments [113]. The oxidation of nanotubes was performed with thermally assisted oxidation in concentrated $\mathrm{HNO}_{3}$ for different amounts of 
time ranging from $0 \mathrm{~h}$ to $24 \mathrm{~h}$ in $4 \mathrm{~h}$ steps. For the oxidation of $4 \mathrm{~g}$ nanotubes $500 \mathrm{ml}$ of $\mathrm{HNO}_{3}$ solution was used. After oxidation, samples were washed with deionized water until neutral $\mathrm{pH}$ was reached, then dried in air at $353 \mathrm{~K}$ overnight. Two other sample series were also created, where the oxidation process was preceded or followed by a Soxhlet-extractor enhanced acetone washing, which series were denoted as "Before Washed" carbon nanotubes (CNT-BW) or "After Washed" carbon nanotubes (CNT-AW), respectively. Nanotubes prepared without acetone washing were denoted as "Not Washed" carbon nanotubes (CNTNW). For the preparation of washed samples $4-4 \mathrm{~g}$ of nanotubes were placed into a shell made of filter paper. Nanotubes were washed with $500 \mathrm{ml}$ of acetone for $48 \mathrm{~h}$ in the Soxhlet extractor, then dried at $353 \mathrm{~K}$ overnight.

Nanotubes were modified with the wet impregnation method similar to the method described for MC samples in section 5.1.3. $22 \mathrm{mg}$ of Pd-acetate was dissolved in $100 \mathrm{ml}$ of toluene, then $200 \mathrm{mg}$ of CNT was added to the solution. The mixture was sonicated in an ultrasonic bath $(80 \mathrm{~W}$ ) for $15 \mathrm{~min}$ to disperse nanotubes, and then mixed for $24 \mathrm{~h}$ at room temperature. The nanotubes were separated from the solvent by centrifugation, then dried in air at $353 \mathrm{~K}$ overnight.

For the formation of nanoparticles, two slightly different approaches were chosen. In the first method samples were annealed in air at $485 \mathrm{~K}$ for $2 \mathrm{~h}$ and $653 \mathrm{~K}$ for $1 \mathrm{~h}$ to form an impregnated intermediate for further processing. Intermediate samples were then divided into three parts, where the first was kept as reference, while the second and third parts were subjected to a second annealing process in air or $\mathrm{N}_{2}-\mathrm{H}_{2}$ gas $\left(10: 1,330 \mathrm{~cm}^{3} / \mathrm{min}\right)$ for $3 \mathrm{~h}$. Samples annealed in air or $\mathrm{N}_{2}-\mathrm{H}_{2}$ gas get the "PdO-“" or "Pd-" prefixes, respectively, while the "-380" suffix will denote the second calcination process when applicable.

The second method only utilized a single annealing process in nitrogen $\left(300 \mathrm{~cm}^{3} / \mathrm{min}\right)$ with identical temperatures and times as described in the first method to form metallic $\mathrm{Pd}$ nanoparticles. Pd-impregnated samples were denoted as Pd-CNT-BW, Pd-CNT-NW and PdCNT-AW. A simplified graphical schematic is presented in Figure 5.12. to help better understand the changes made by various preparation steps on the surface environment of carbon nanotubes. The schematic is applicable for samples prepared with the second impregnation method only. 


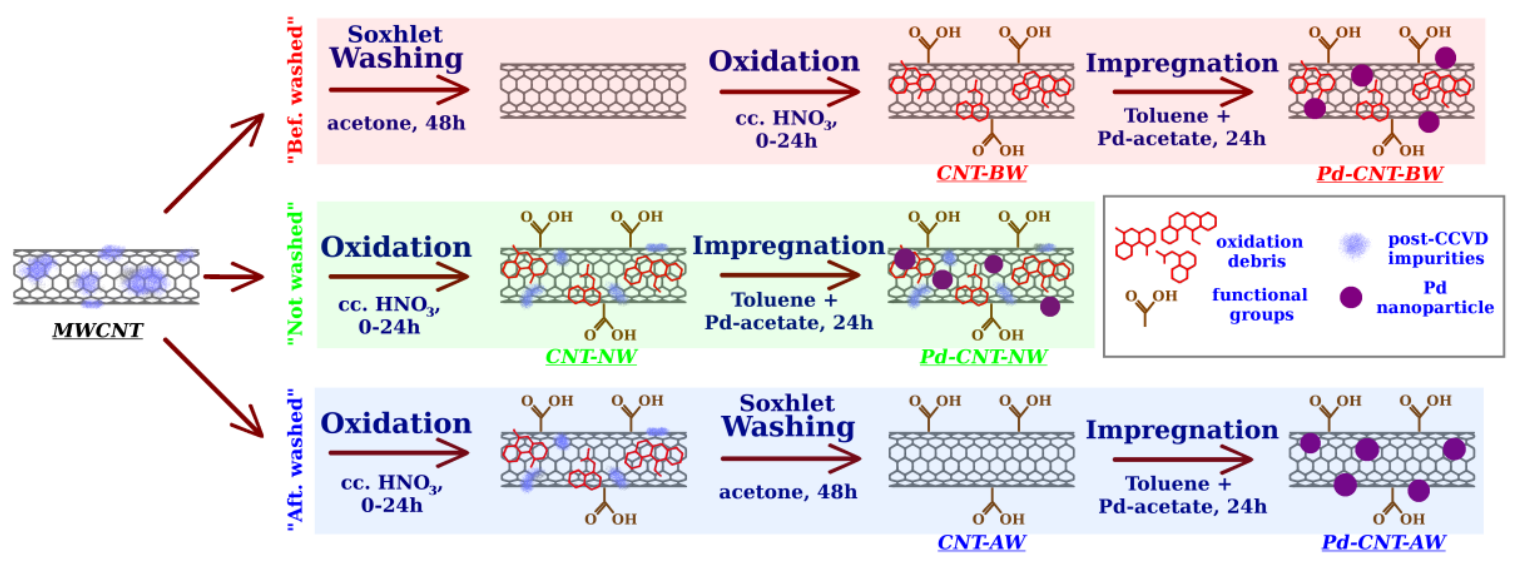

Figure 5.12: A graphical overview of the effects of various sample preparation steps on the surface environment of carbon nanotubes

\subsubsection{Characterization of palladium and palladium oxide modified "not-washed" oxidized carbon nanotubes}

In order to establish an understanding how metallic and oxide nanoparticles behave on oxidized CNTs, a basic series of not washed nanotubes with oxidation times $0,4,8,12$ and $16 \mathrm{~h}$ were prepared as first step. The shorter series was considered to be enough to learn the differences in particle size for oxide and metallic nanoparticles supported on identically treated CNTs.

\subsection{2.a. X-ray diffraction}



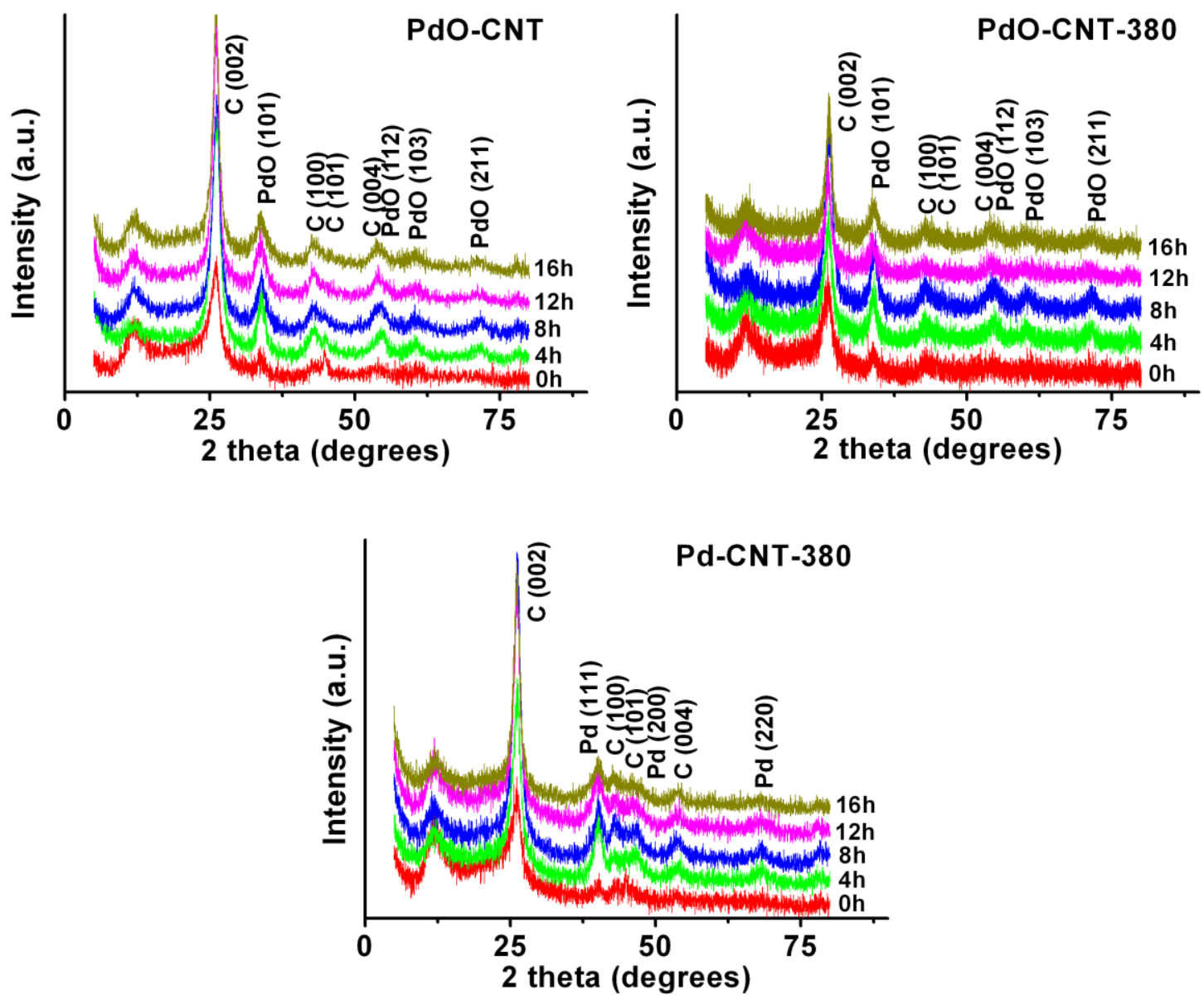

Figure 5.13. XRD patterns of Pd and PdO modified carbon nanotubes.

The formation of $\mathrm{Pd}$ and $\mathrm{PdO}$ nanoparticles on CNTs were followed by XRD. Diffractograms of all sample series are presented in Fig. 5.13. Peaks corresponding to the crystal lattice planes of PdO and Pd with Miller indexes of (101) (112) (103) (211) and (111) (200) (220) (311) at d-spacing values of 2.63, 1.66, 1.53, 1.31 and 2.24, 1.93, 1.37, $1.17 \AA$, respectively are observable. Crystallite sizes were calculated using Scherrer's equation from PdO (101) and Pd (111) peaks. Data obtained is tabulated in Table 5.3.

Table 5.3. CNT supported Pd and PdO crystal diameters (in $\mathrm{nm}$ ) calculated using Scherrer's equation for different oxidation times.

\begin{tabular}{lccccc}
\hline & $0 \mathrm{~h}$ & $4 \mathrm{~h}$ & $8 \mathrm{~h}$ & $12 \mathrm{~h}$ & $16 \mathrm{~h}$ \\
\hline PdO-CNT & 5.9 & 5.6 & 4.9 & 3.7 & 3.2 \\
PdO-CNT-380 & 7.2 & 6.4 & 5.4 & 4.8 & 4.2 \\
Pd-CNT-380 & 7.2 & 4.6 & 3.9 & 3.2 & 3.0 \\
\hline
\end{tabular}


An interesting connection is observable between functionalization time and calculated crystallite size. Calculated crystal diameters on functionalized PdO-CNT samples exhibited a gradual decrease starting from $5.6 \mathrm{~nm}$ to $3.2 \mathrm{~nm}$. A similar trend is observable for samples annealed a second time, where crystal diameters on functionalized CNTs gradually decreased from $6.4 \mathrm{~nm}$ to $4.2 \mathrm{~nm}$ and $4.6 \mathrm{~nm}$ to $3.0 \mathrm{~nm}$ at sample series PdO-CNT-380 and Pd-CNT380, respectively. Crystallite size was highest on non-functionalized CNT supported PdOCNT, PdO-CNT-380 and Pd-CNT-380 samples with diameters of $5.9 \mathrm{~nm}, 7.2 \mathrm{~nm}$ and 7.2 $\mathrm{nm}$, respectively. These results indicate, that there might be an inverse relation between functionalization time and crystallite size.

\subsection{2.b. Transmission electron microscopy}
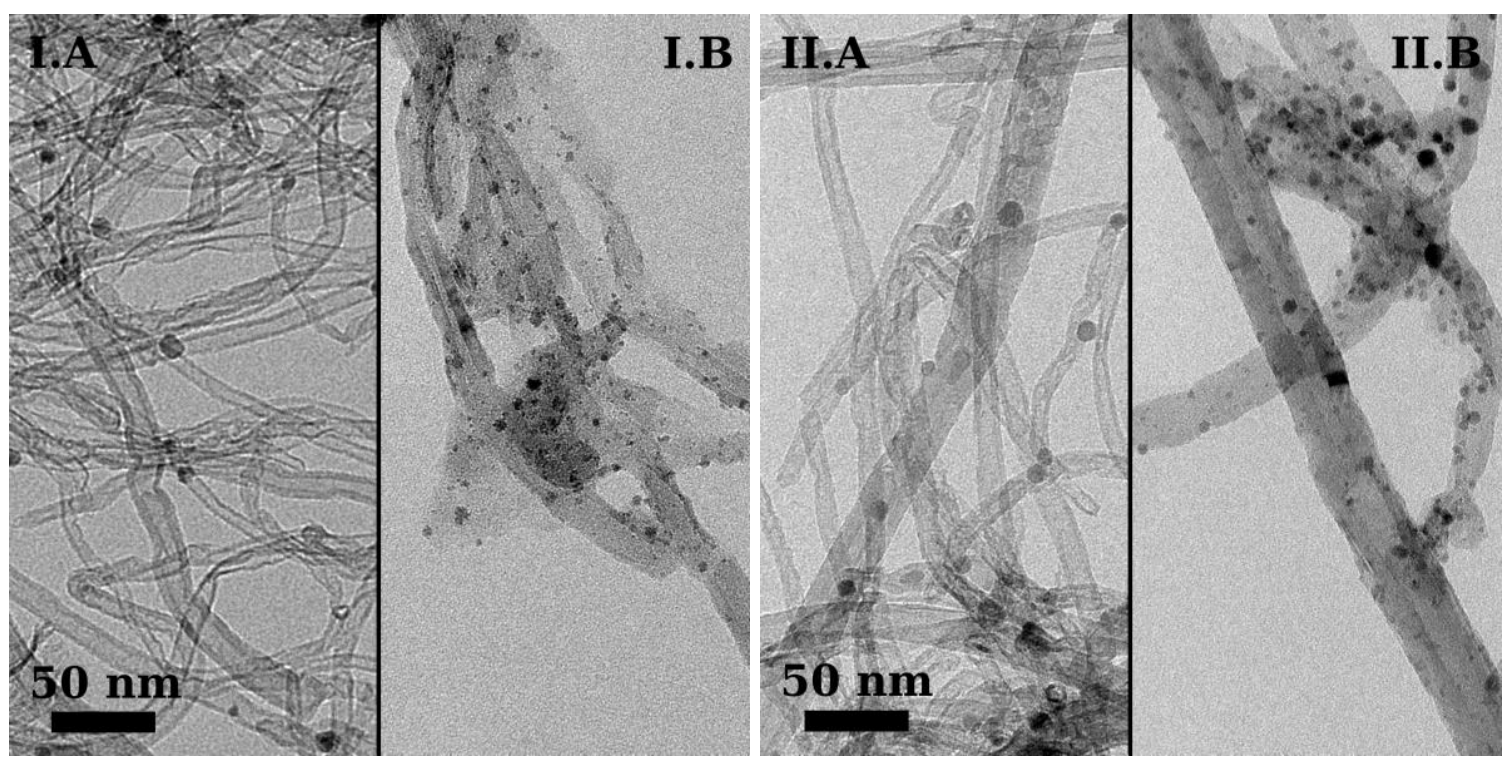


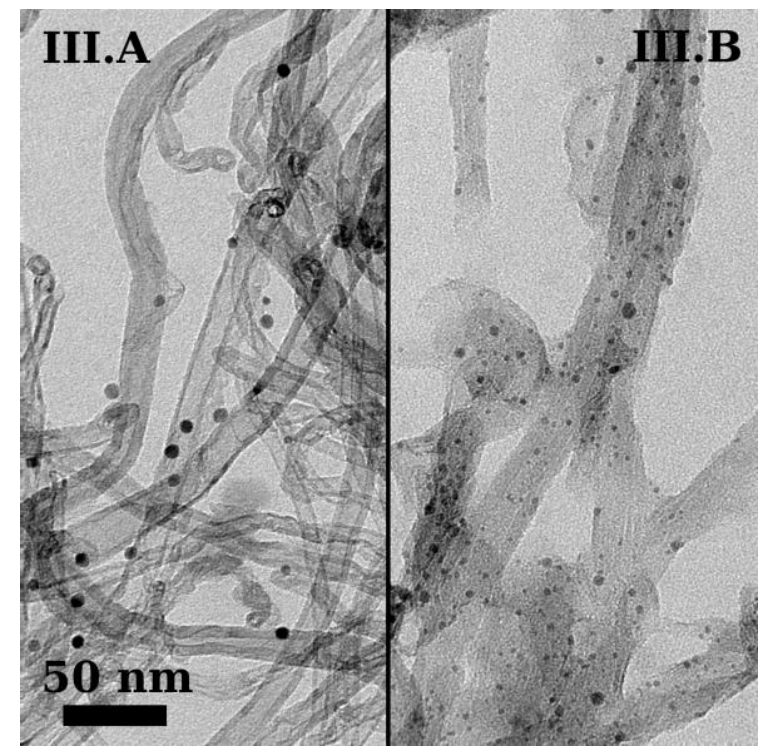

Figure 5.14. TEM micrographs of PdO and Pd modified CNTs. I: PdO-CNT, II: PdO-CNT-380 and III: Pd-CNT-380. Micrographs with captions " $A$ " and " $B$ " represent oxidation times $0 \mathrm{~h}$ and $16 \mathrm{~h}$, respectively.

TEM micrographs were captured of all nanoparticle modified samples. A clear difference is visible in both nanotube structure and nanoparticle size between not oxidized and oxidized samples presented (Fig 5.14.). Not oxidized nanotubes exhibit undamaged structures with nanoparticles supported on the sidewalls, whereas nanotubes oxidized for 16 h clearly show damaged walls and nanoparticles with considerably smaller size. To verify the results of XRD analysis the size of nanoparticles were manually scaled on TEM micrographs. The resulting data is tabulated in Table 5.4.

Particles supported on not oxidized samples exhibit an average diameter of $8.0 \mathrm{~nm}, 8.5$ $\mathrm{nm}$ and $6.4 \mathrm{~nm}$ for samples PdO-CNT, PdO-CNT-380 and Pd-CNT-380, respectively. Particles supported on oxidized samples show a gradually decreasing average diameter as functionalization time increases, starting from $7.5 \mathrm{~nm}, 6.8 \mathrm{~nm}$ and $5.1 \mathrm{~nm}$ at $4 \mathrm{~h}$ to $5.6 \mathrm{~nm}$, $4.3 \mathrm{~nm}$ and $3.1 \mathrm{~nm}$ at $16 \mathrm{~h}$ for PdO-CNT, PdO-CNT-380 and Pd-CNT-380 series, respectively. These results are in agreement with results obtained for crystallite sizes in the XRD study, whereas an inverse proportionality is observed between functionalization time and crystallite/particle size.

Interestingly, $\mathrm{PdO}$ particles supported on oxidized samples annealed for a second time (PdO-CNT-380) are smaller than PdO particles found on PdO-CNT series. This phenomenon could be attributed to fact that particles seen on TEM micrographs can be built up from several smaller crystallites, which form agglomerates seen as a single nanoparticle. These 
smaller crystallites can then sinter at higher temperatures essentially eliminating the intercrystallite voids making the overall new particle smaller in size.

Table 5.4. Pd and PdO nanoparticle diameters (in $\mathrm{nm}$ ) scaled manually on TEM micrographs for different CNT oxidation times.

\begin{tabular}{lccccc}
\hline & $0 \mathrm{~h}$ & $4 \mathrm{~h}$ & $8 \mathrm{~h}$ & $12 \mathrm{~h}$ & $16 \mathrm{~h}$ \\
\hline PdO-CNT & $8.0 \pm 1.8$ & $7.5 \pm 2.3$ & $6.9 \pm 2.4$ & $5.2 \pm 1.9$ & $5.6 \pm 2.5$ \\
PdO-CNT-380 & $8.5 \pm 2.2$ & $6.8 \pm 1.7$ & $5.7 \pm 2.3$ & $4.8 \pm 1.9$ & $4.3 \pm 1.5$ \\
Pd-CNT-380 & $6.4 \pm 1.5$ & $5.1 \pm 1.7$ & $4.0 \pm 1.7$ & $3.4 \pm 1.3$ & $3.1 \pm 1.1$ \\
\hline
\end{tabular}

This hypothesis is supported by the comparison of particle size obtained by TEM $\left(\mathrm{d}_{\text {TEM }}\right)$ measurements and crystallite sizes calculated from XRD patterns $\left(\mathrm{d}_{\text {Sch }}\right)$ (Figure 5.16. A), B)). While the difference between $\mathrm{d}_{\text {Sch }}$ and $\mathrm{d}_{\text {TEM }}$ was $\sim 2 \mathrm{~nm}$ for PdO-CNT samples, this difference is essentially eliminated for PdO-CNT-380 samples. Average crystallite sizes calculated for PdO-CNT-380 also tend to be higher than their PdO-CNT supported counterparts, which alone can prove the sintering of crystallites.

\subsection{2.c. Raman Spectroscopy}

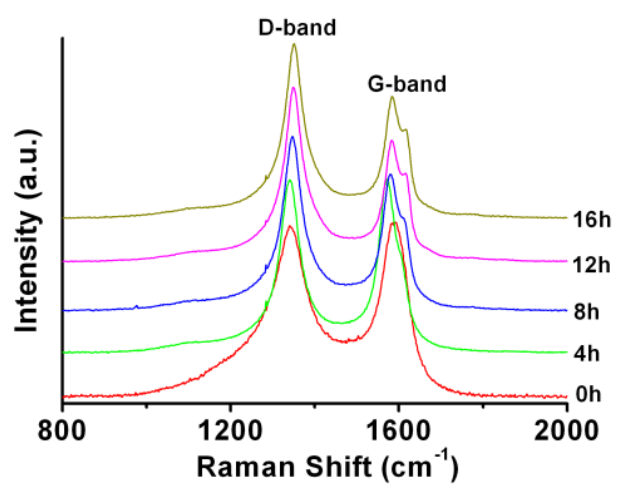

Figure 5.15. Raman spectra of non-impregnated carbon nanotubes oxidized for 0-16 h.

Raman spectra were collected on all non-impregnated CNT samples. The main two features of the spectra are the $D$ and $G$ bands found at $\sim 1349 \mathrm{~cm}^{-1}$ and $\sim 1586 \mathrm{~cm}^{-1}$, respectively. The intensity of the disorder-induced D-band is generally associated with the amount of defects (more precisely, with the amount of $\mathrm{sp}^{3}$ hybridized phonon scattering sites) in the nanotubes, while the G-band is attributed to the tangential lattice vibrations of $\mathrm{sp}^{2}$ bonded carbon atoms. G/D ratios are plotted alongside with particle diameter data in Fig 5.16. As it is seen, there is a correlation between functionalization time, G/D ratios and 
nanoparticle size: the smaller the G/D ratio, the smaller the obtained average nanoparticle diameter, regardless of nanoparticles being oxide or metallic. As first assumption, the growing amount of surface defect sites were thought to be the main governing factor behind sintering and aggregation inhibition, but as later studies reveal in Section 5.2.3, there is a more complex process behind these phenomena.
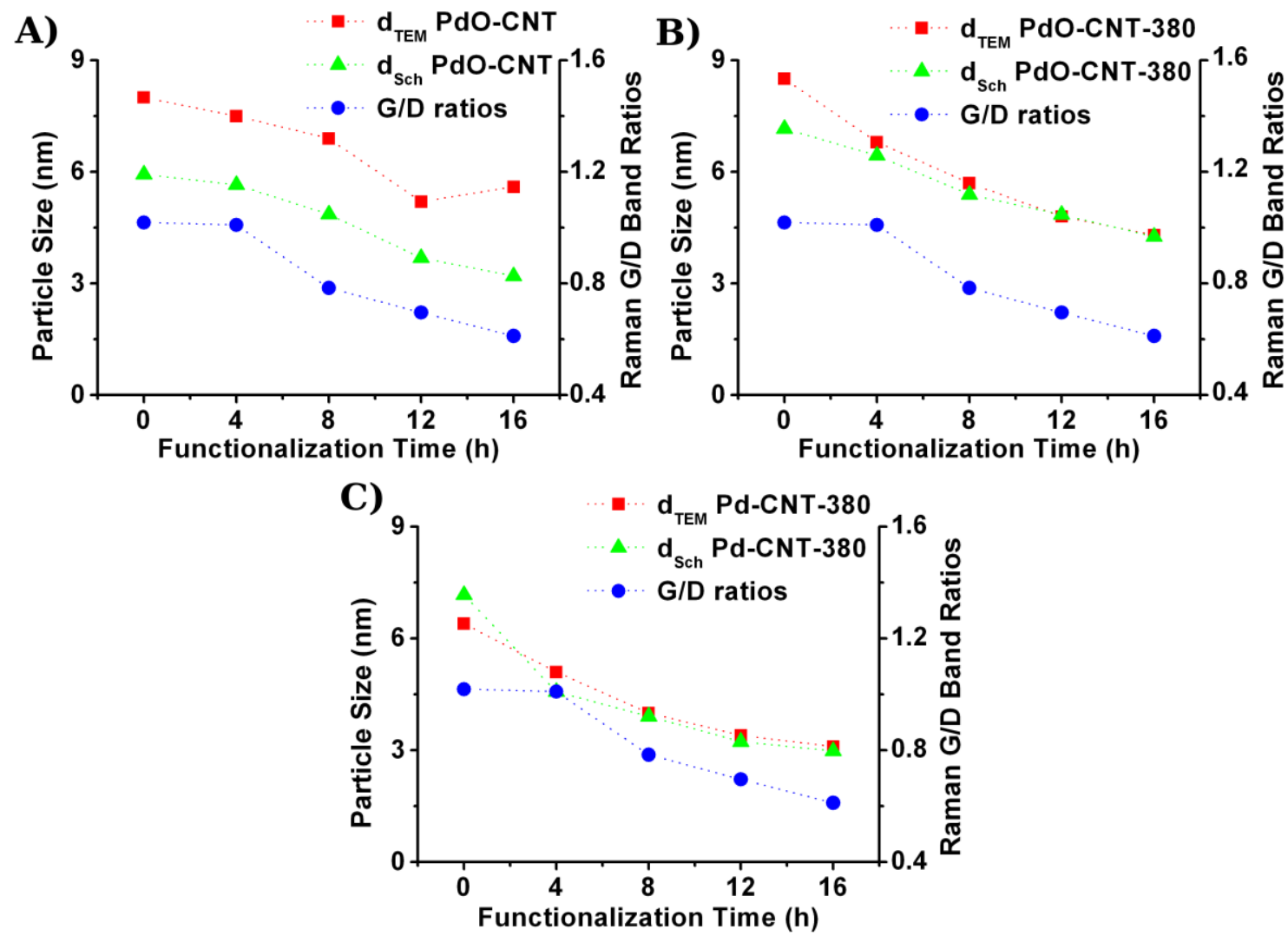

Figure 5.16. Comparison of particle diameters obtained for different sample series. Raman G/D ratios, particle diameters $\left(d_{\text {TEM }}\right)$ and crystallite diameters $\left(d_{\text {Sch }}\right)$ are plotted for $\left.\mathrm{A}\right)$ PdO-CNT, B) PdO-CNT-380 and C) Pd-CNT-380 sample series.

\subsubsection{Characterization of palladium modified oxidized carbon nanotubes prepared with Soxhlet-extractor enhanced acetone washing.}

In section 5.2.2 a correlation between oxidation time, Raman G/D bands and particle size has been established. As no difference was found between the general behavior of oxide and metallic nanoparticles on oxidized CNTs, only metallic palladium modified nanotubes were examined at later experiments. The full series of "Not Washed", "After Washed" and "Before Washed" were prepared for these tests with oxidation times up to $24 \mathrm{~h}$. In this part the various effects of post-CCVD impurities, oxidation debris, functional groups and structural defects have been studied.

\subsection{3.a. Raman Spectroscopy}



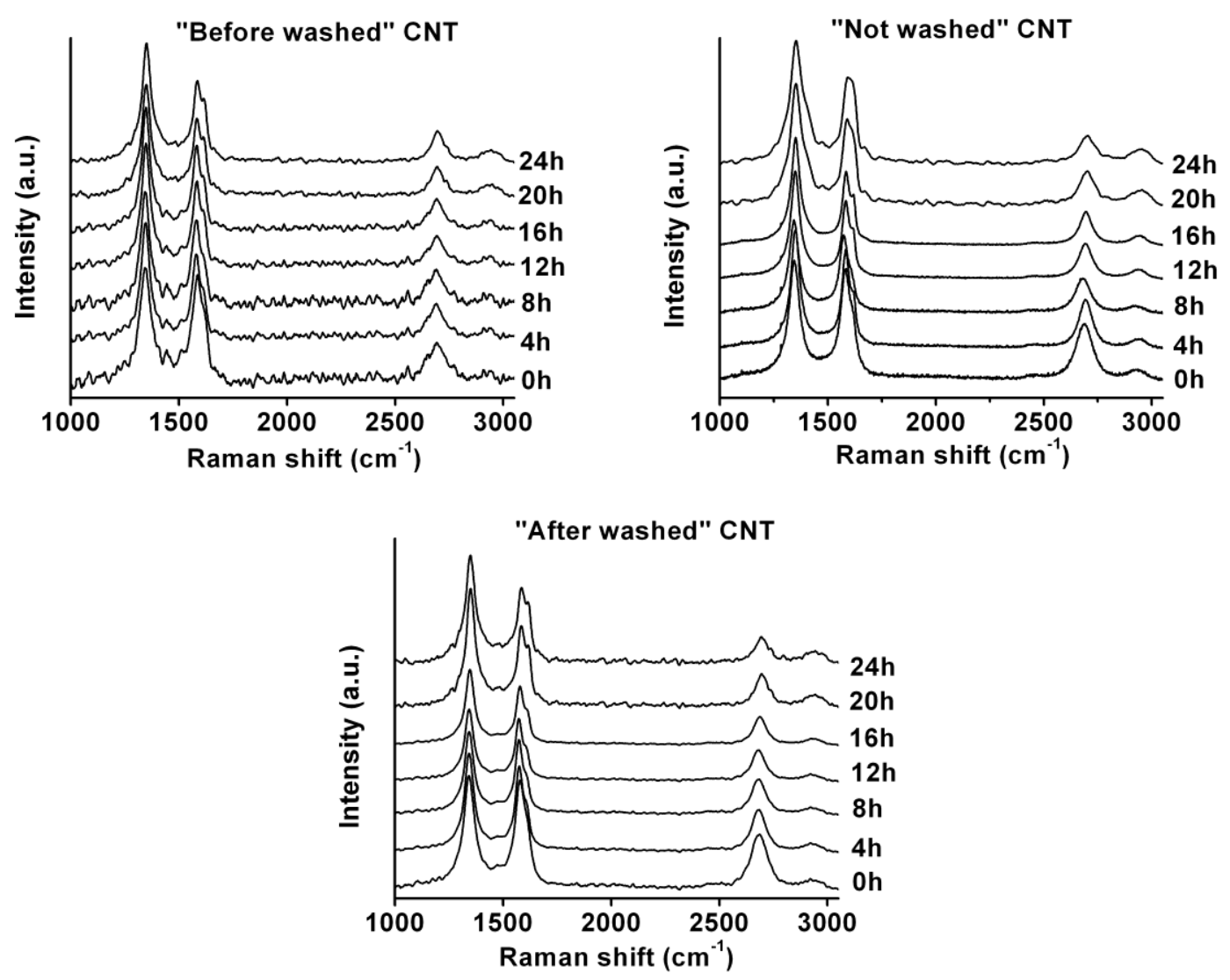

Figure 5.17. Raman spectra of carbon nanotubes oxidized from 0 to $24 \mathrm{~h}$. Spectra are divided into 3 sample series depending on the usage of Soxhlet extracor enhanced aceteone washing.

Raman spectra were collected for samples series CNT-BW, CNT-NW and CNT-AW (Fig 5.17). As discussed in the section 5.2.2.c, the ratio of Raman D and G band were examined to reveal structural differences between samples (Fig 5.18). In case of CNT-NW series the G/D ratio gradually decreased from 0.95 to 0.71 from $0 \mathrm{~h}$ to $24 \mathrm{~h}$, respectively, which is a $25 \%$ overall drop. This is in agreement with results discussed for $\mathrm{Pd}$ and $\mathrm{PdO}$ modified CNTs in section 5.2.2.c. Interestingly, if CNTs were washed before or after oxidation, an immediate $20 \%$ drop is visible after $4 \mathrm{~h}$ of oxidation, which is followed by a much slower graduate decrease if any. Values of CNT-AW's slowly decrease from 0.78 to 0.76 from $4 \mathrm{~h}$ to $16 \mathrm{~h}$, reaching 0.70 at even longer functionalization times. After the $\sim 20 \%$ drop in the first 4 hours, the CNT-BW samples show a slow G/D ratio decrease from 0.75 to 0.70 between $4-16$. The G/D ratio is independent of the washing process if functionalization is performed for longer than $20 \mathrm{~h}$.

Both CNT-NW and CNT-BW series exhibited a clearly distinguishable G/D ratio decrease between $4 \mathrm{~h}$ and $16 \mathrm{~h}$ of oxidation, while CNT-AW series showed almost constant G/D ratios in the same range. This drop can thus be attributed to the formation of oxidation 
debris and defect sites, as the drop only occurs on samples not washed after oxidation. If functional groups played a significant role in G/D ratio changes, the same drop would be present on CNT-AW series also. G/D ratios are also found to be lower for CNT-BW samples if compared to then for CNT-NW. This can be the result of the washing process, as washing applied before oxidation process can only remove already present impurities, namely postCCVD ones. This means that the presence of post-CCVD impurities have a significant effect on the oxidation process.

Interestingly, G/D ratios do not seem to be affected by post-CCVD impurities, as washed and not washed non-oxidized $(0 \mathrm{~h})$ samples exhibited virtually identical ratios. This could indicate that the amount of impurities left from the synthesis procedure were only present in rather small quantities. Nevertheless, this small amount is enough to alter surface properties of CNTs as will be evidenced by nitrogen adsorption measurements later.

As discussed previously, a steady decrease in G/D ratio for the CNT-NW series is apparent, which could probably be attributed to the formation of both oxidation debris and functional groups. The G/D ratio of CNT-AW samples on the other hand does not show such clear decrease. After a sudden drop at $4 \mathrm{~h}$ of oxidation the changes in ratio is insignificantly small up until $16 \mathrm{~h}$. This difference between G/D ratios of CNT-NW and CNT-AW series comes from the washing process only, as no other treatments were present after oxidation. The washing process thus caused the removal of oxidation debris regardless of functionalization time, leaving only functional groups unaffected by the washing on the surface. These groups do not seem to alter the G/D ratio of nanotubes.

It must be noted, that the G/D ratios of CNT-AW samples were found to be constantly higher up until $20 \mathrm{~h}$ of oxidation. This may be due to the fact that CNT-AW series were only washed after the oxidation process, thus post-CCVD impurities were still present on the surface during the acidic treatment. Since the graphitic structure of nanotubes is less prone to oxidation then the structurally amorphous impurities, it can be assumed that postCCVD impurities are more preferentially consumed by the nitric acid, thus exhibiting a shielding effect for nanotubes walls. This resulted in a slightly smaller number of defective sites on the walls of the carbon nanotubes compared to CNT-BW samples, where no impurities were present at the time of oxidation and the nitric acid attack could be fully directed at the CNT walls.

Samples oxidized for $20 \mathrm{~h}$ or longer exhibited virtually identical G/D ratios, which could imply that the concurring defect-generating and defect-consuming processes reached an equilibrium. These results thus indicate, that while oxidation debris greatly affects 
detected Raman signals, post-CCVD impurities and anchored functional groups barely alter the obtained spectra even though they can have a major impact on other attributes of the samples.

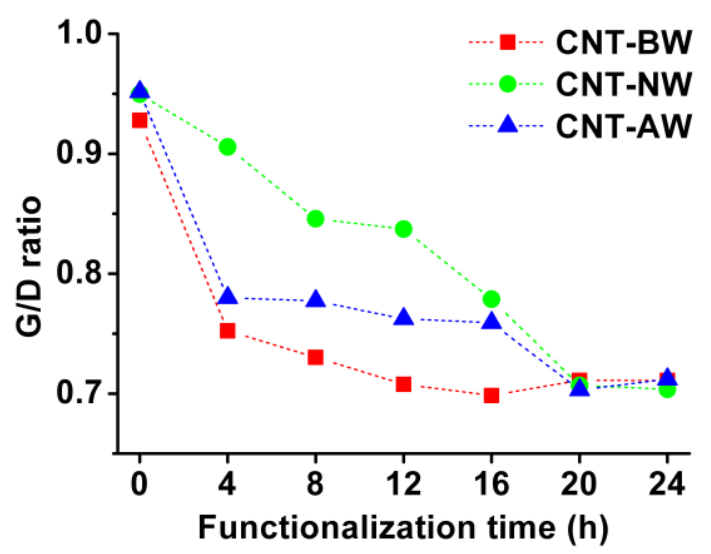

Figure 5.18. Raman G/D band ratios of multiwalled carbon nanotube samples after different functionalization durations (4-24 h) in the case of "Non Washed", "Before Washed" and "After Washed" samples (lines are guide to the eye).

\subsection{3.b. Nitrogen gas adsorption}

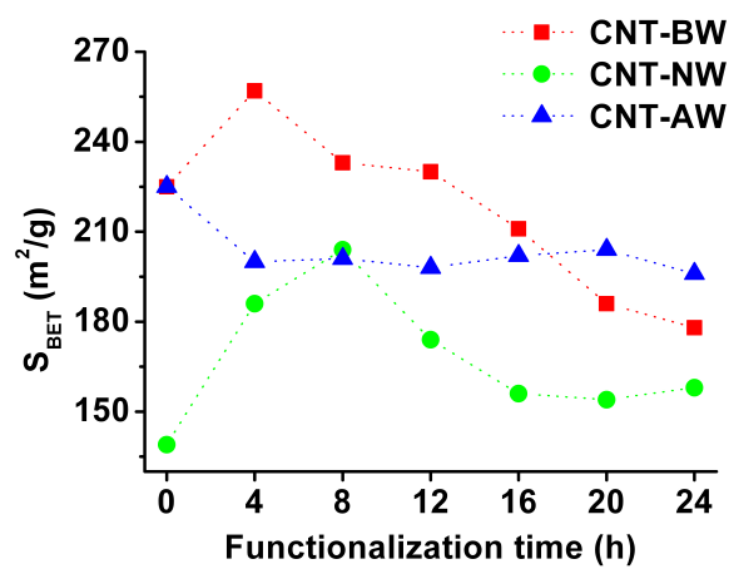

Figure 5.19: Specific surface area of carbon nanotubes subjected to different washing processes as a function of the oxidation time. Lines are guides for the eye only.

Nitrogen gas adsorption isotherms were collected for all three sample series. Specific surface area $\left(\mathrm{S}_{\mathrm{BET}}\right)$ values were calculated using the well-known Brunauer-Emmett-Teller (BET) method. Calculated data were plotted in Figure 5.19, where the effects of acetone washing and functionalization are evident.

In case of CNT-BW samples, $\mathrm{S}_{\mathrm{BET}}$ value rises from $225 \mathrm{~m}^{2} \cdot \mathrm{g}^{-1}$ to $257 \mathrm{~m}^{2} \cdot \mathrm{g}^{-1}$ at $4 \mathrm{~h}$ of oxidation, then it shows a steady drop up to $24 \mathrm{~h}$ functionalization where it reaches $178 \mathrm{~m}^{2} \cdot \mathrm{g}^{-}$ 1. The initial $S_{\text {BET }}$ value of the CNT-NW sample is $140 \mathrm{~m}^{2} \cdot \mathrm{g}^{-1}$, then it increases to $205 \mathrm{~m}^{2} \cdot \mathrm{g}^{-1}$ 
after $8 \mathrm{~h}$ functionalization and decreases again to $155 \mathrm{~m}^{2} \cdot \mathrm{g}^{-1}$ by $16 \mathrm{~h}$. There is no significant change in specific surface area after $16 \mathrm{~h}$ of functionalization for CNT-NW samples. CNTAW samples do not display the tendencies showed by the CNT-BW and CNT-NW series. Rather, their $\mathrm{S}_{\text {BET }}$ of $225 \mathrm{~m}^{2} \cdot \mathrm{g}^{-1}$ drops to $200 \mathrm{~m}^{2} \cdot \mathrm{g}^{-1}$ at $4 \mathrm{~h}$ and remains fairly constant at this value at longer functionalization times.

The sudden rise for both CNT-NW and CNT-BW series can be attributed to the preferential oxidation of CNT caps in the first few hours of oxidation, as higher C-C bonding curvature and pentagon concentration make these sites more prone to oxidation [186]. The steady drop of $\mathrm{S}_{\mathrm{BET}}$ values found for longer oxidation times can be attributed to the forming oxidation debris that at least partially block interstitial and intratube adsorption sites as the amount of debris increases with oxidation time. Oxidation debris is believed to be mainly composed of peeled off oxidized graphene "islands" that later form amorphous carbon [281,282].

CNT-NW and CNT-BW (or CNT-AW) non-oxidized samples showed great difference in $S_{\mathrm{BET}}$ values, whereas the only difference between the preparation methods of the two samples was the addition of the acetone washing performed in the Soxhlet-extractor. The $\sim 40 \%$ sudden leap from $140 \mathrm{~m}^{2} / \mathrm{g}$ to $225 \mathrm{~m}^{2} / \mathrm{g}$ in $\mathrm{S}_{\mathrm{BET}}$ after the washing thus clearly indicate that adsorption sites were vacated by the process. Since the only type of contamination present on non oxidized samples is post-CCVD impurities, the leap in $\mathrm{S}_{\mathrm{BET}}$ can only be explained by the removal of such impurities. Interestingly small amounts of post-CCVD impurities were not found to significantly alter Raman spectra, yet such amount are enough to greatly affect surface properties. This difference in $\mathrm{S}_{\mathrm{BET}}$ values does not disappear between CNT-BW and CNT-NW samples for longer functionalization times, which suggests a similar adsorption site blocking mechanism for the two series, namely the formation of oxidation debris during functionalization. The slight shift of peak maximum position to longer oxidation times of CNT-NW samples compared to CNT-BW samples is due to the presence of post-CCVD contamination, which could reduce the speed of the cap opening.

Oxidized CNT-AW series showed fairly constant $S_{\text {BET }}$ value of $\sim 200 \mathrm{~m}^{2} / \mathrm{g}$ which is a result of the removing of oxidation debris by the washing process. This means that functional groups and defect sites forming as a result of oxidation do not seriously alter the specific surface area of the nanotubes themselves, not even after prolonged acidic treatment. Rather, differences in the $\mathrm{S}_{\mathrm{BET}}$ values are related to the blockage of various pores and adsorption sites by the debris formed during the oxidation process. Results for non-oxidized washed samples 
also indicate that post-CCVD impurities greatly alter $S_{\mathrm{BET}}$ values even in small quantities, even though they are not detectable by Raman spectroscopy.

\subsection{3.c. X-Ray diffraction}

The formation of metallic Pd was also confirmed by powder XRD measurements, where peaks characteristic for fcc Pd (111) and Pd (200) reflections were detected on all Pd modified samples at d-spacing values of 2.24 and $1.93 \AA$, respectively. Calculation of crystallite sizes with Scherrer's equation was not possible due to the pronounced peak broadening typical in this particle size regime. XRD patterns are presented in Figure 5.20.

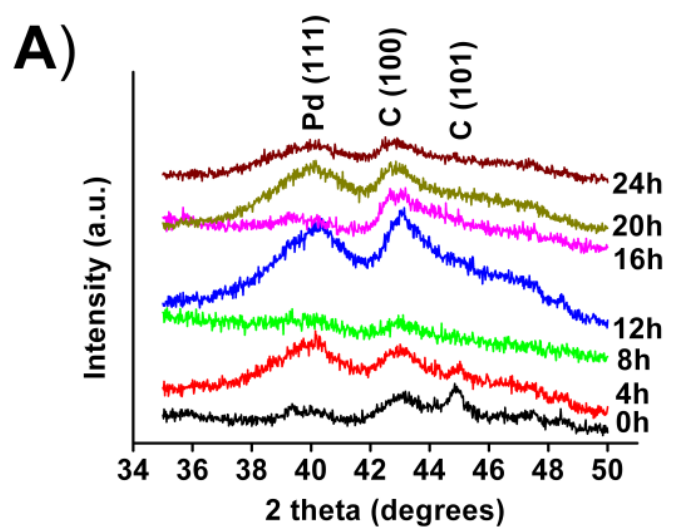

B)
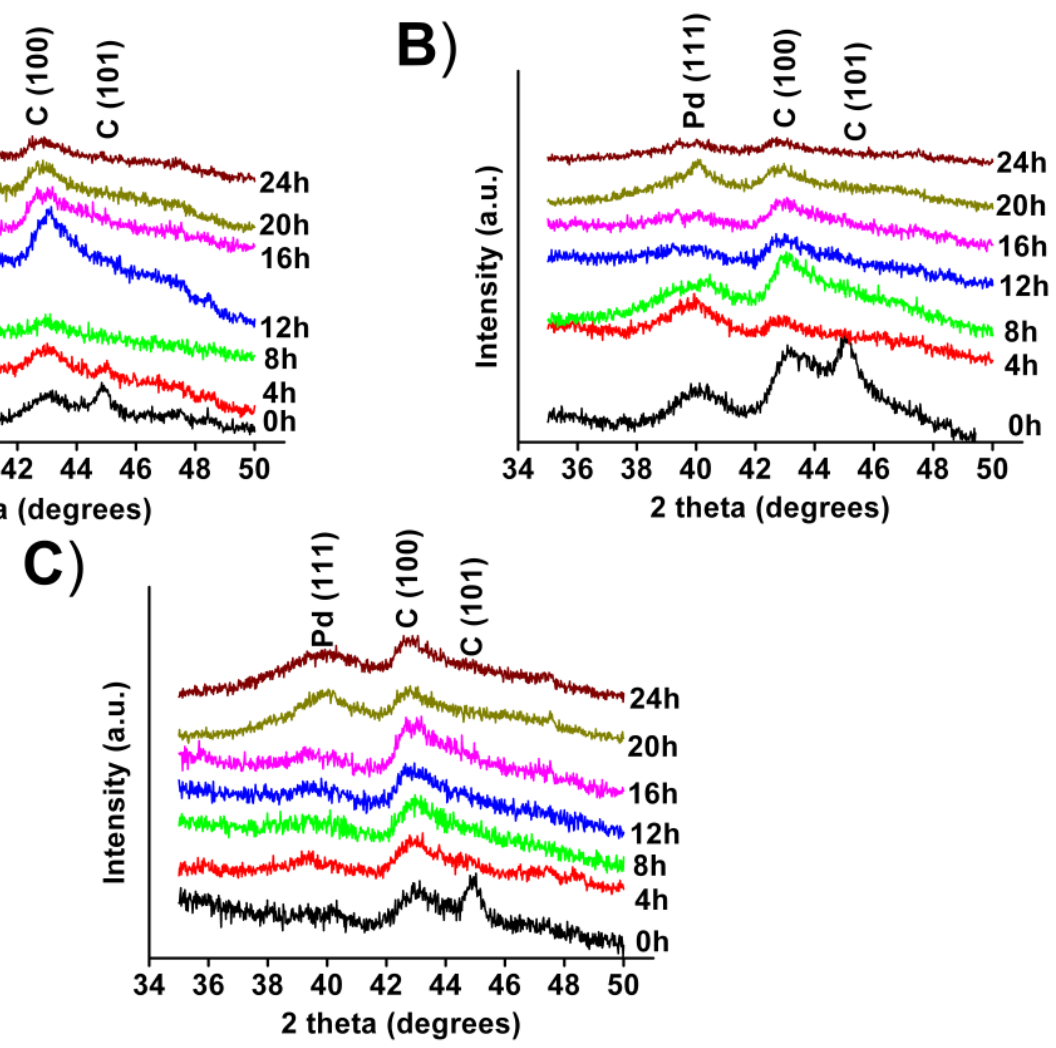

Figure 5.20. XRD patterns of palladium modified A) "Before Washed", B) "Not Washed" and C) "After Washed" sample series

\subsection{3.d. Transmission electron microscopy}




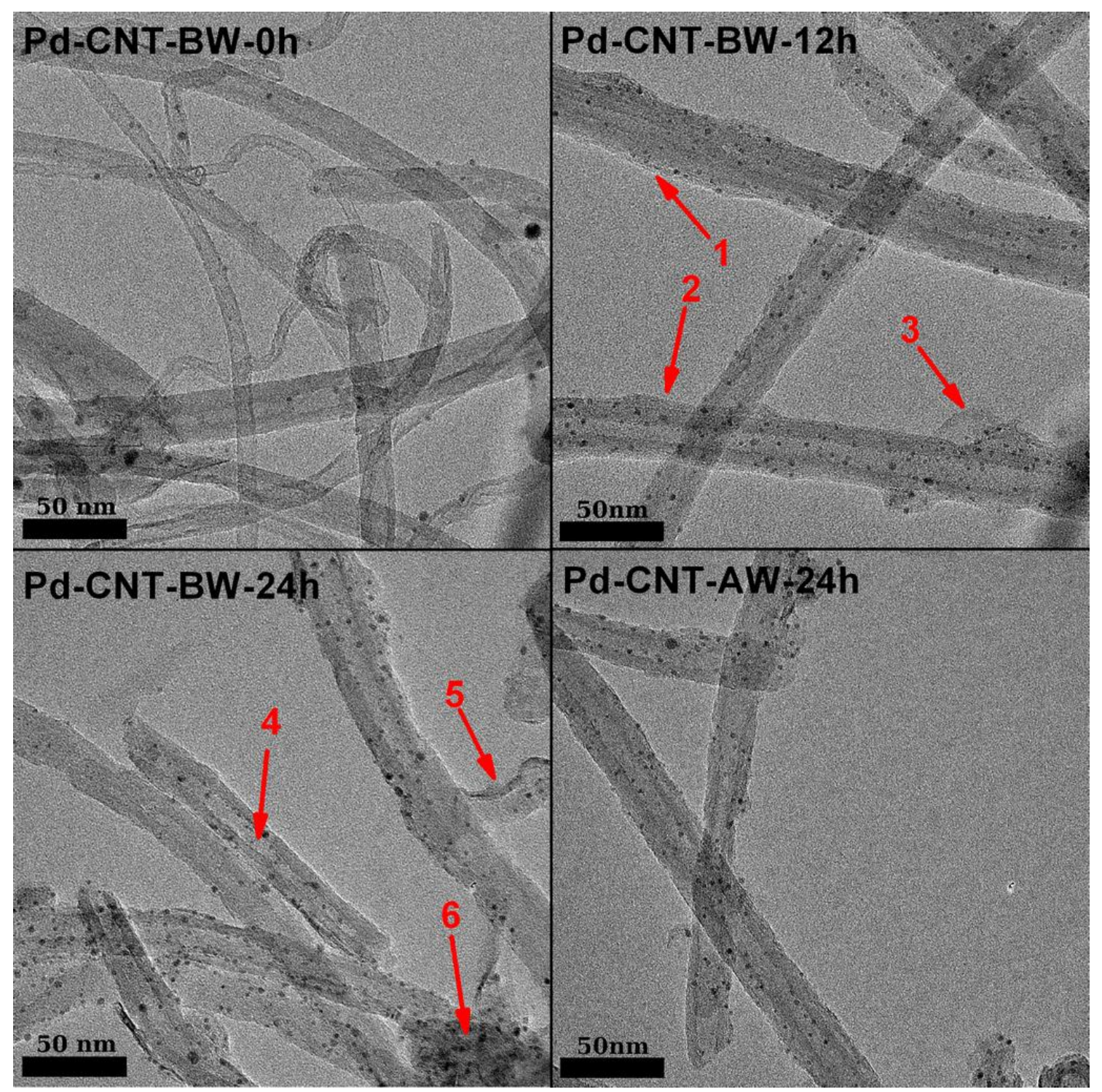

Figure 5.21. TEM micrographs of Pd/CNT samples from the "Before Washed" series with nanotube supports oxidized for $\mathbf{0 , 1 2}$ and $24 \mathrm{~h}$. Numbered arrows highlight distinct features of oxidized samples, such as surface coverage (1,2), loose layers of CNT (3), short nanotube pieces (4), CNT fragments (5) and amorphous content (6). The surface of "After Washed" nanotube sample oxidized for 24 h contains no debris or other contaminants.

All Pd modified carbon nanotubes were examined by transmission electron microscopy. TEM micrographs of selected samples are presented in Figure 5.21. All the particles were found to be evenly dispersed on nanotubes, no separate particles were found. The size of particles were scaled manually, calculated mean diameters and standard deviations are tabulated in Table 5.5.

Non oxidized $\left(\begin{array}{ll}0 & \mathrm{~h}\end{array}\right)$ acetone washed nanotubes exhibited intact surfaces, no contamination of any sort is visible. After $12 \mathrm{~h}$ of oxidation however evidence of surface coverage (Arrows 1 and 2, Fig. 5.21) and some loose layers (Arrow 3, Fig. 5.21) is apparent, which is most probably the oxidation debris formed during functionalization, as discussed 
earlier. After $24 \mathrm{~h}$ of oxidation amorphous carbon content (Arrow 6, Fig. 5.21) and CNT fragments (Arrow 5, Fig. 5.21) appear in the samples as a result of extensive oxidative treatment. Nanotubes are also known to break up into shorter pieces after long functionalization times, as evidenced by the Pd-CNT-BW-24h sample (Arrow 4, Fig. 5.21). In contrast to $12 \mathrm{~h}$ or $24 \mathrm{~h}$ oxidized "Before Washed samples, "Pd-CNT-AW-24h sample showed surfaces clean of contamination, which is an independent verification for the successful removal of oxidation debris by the Soxhlet-extractor washing. The roughness of nanotubes surfaces is most likely the result of surface defects generated by the prolonged exposure to nitric acid.

The average Pd particle diameter was $4.8 \mathrm{~nm}$ for Pd-CNT-NW samples without functionalization. This value decreased to $3.6 \mathrm{~nm}$ at $4 \mathrm{~h}, 2.9 \mathrm{~nm}$ at $8 \mathrm{~h}$ and reached $2.3 \mathrm{~nm}$ at $12 \mathrm{~h}$, then remained fairly constant up to $24 \mathrm{~h}$ functionalization time. The Pd-CNT-BW and Pd-CNT-AW samples featured an average particle diameter of $2.4 \mathrm{~nm}$ and $2.8 \mathrm{~nm}$, respectively independent of the functionalization duration. The standard deviation of the particle diameter was below $1 \mathrm{~nm}$ for most of the samples. However, Pd nanoparticles supported on non-functionalized carbon nanotubes showed slightly higher standard deviation of $0.8-2.3 \mathrm{~nm}$.

Table 5.5. Average diameter and standard deviation of Pd nanoparticles supported on "Before Washed", "Not Washed" and "After Washed" sample series.

\begin{tabular}{cccc}
\hline Oxidation time (h) & \multicolumn{3}{c}{ Particle diameter $(\mathbf{n m})$} \\
\hline & Pd-CNT-BW & Pd-CNT-NW & Pd-CNT-AW \\
0 & $2.7 \pm 0.8$ & $4.8 \pm 2.3$ & $2.7 \pm 0.8$ \\
4 & $2.3 \pm 0.4$ & $3.6 \pm 1.2$ & $2.7 \pm 0.6$ \\
8 & $2.3 \pm 0.4$ & $2.9 \pm 0.9$ & $2.7 \pm 0.7$ \\
12 & $2.2 \pm 0.4$ & $2.3 \pm 0.5$ & $2.8 \pm 0.9$ \\
16 & $2.3 \pm 0.5$ & $2.2 \pm 0.5$ & $2.9 \pm 0.9$ \\
20 & $2.3 \pm 0.7$ & $2.3 \pm 0.6$ & $2.8 \pm 0.6$ \\
24 & $2.6 \pm 0.8$ & $2.2 \pm 0.5$ & $2.9 \pm 0.6$ \\
\hline
\end{tabular}

In the case of CNT-NW samples, oxidation times yielded Pd nanoparticle diameters similar to our earlier observations [16]. The size of the Pd nanoparticles supported on CNTBW and CNT-AW series of carbon nanotubes seem to be entirely insensitive to the functionalization time. It must be noted however, that in case of washed and not washed unfunctionalized samples the mean diameter changed to $2.7 \pm 0.8 \mathrm{~nm}$ from $4.8 \pm 2.3 \mathrm{~nm}$, 
respectively. This indicates a major change in the carbon nanotube surface environment due the removal of post-CCVD impurities during the Soxhlet-extractor washing process. Some difference in average particle diameter and standard deviation is still observable between not washed and washed samples oxidized for $4 \mathrm{~h}$. This could mean that $4 \mathrm{~h}$ of oxidation may not remove all post-CCVD impurities.

Although the changes in Raman G/D intensity ratios were very similar between the CNT-BW and CNT-AW series, the corresponding $S_{\mathrm{BET}}$ values exhibit considerable differences. The dissimilarity revealed by the nitrogen sorption studies however, does not contradict the previously found Raman similarities between the washed samples, rather, it can help understand how the washing process alters the environment of the carbon nanotube surfaces and which types of carbon species contribute to Raman spectra or alter the size of Pd nanoparticles with functionalization time. Nitrogen sorption data of CNT-BW samples indicate that oxidation debris is formed during extended functionalization, which lowers $S_{\mathrm{BET}}$ after $4 \mathrm{~h}$. Post-CCVD impurities influence the nanoparticle size and size distribution as evidenced by the TEM studies of the unfunctionalized samples. The presence of oxidation debris does not seriously alter the size of supported particles. This may be attributed to the fact that oxidation debris does not encompass the available surface of the carbon nanotubes, rather, it accumulates in certain spots like interstitial and intratube pore entrances (nanotube endings). Since nanotubes are impregnated with Pd nanoparticles only after functionalization, the inner surfaces of the CNTs are available for Pd nanoparticle anchoring during impregnation from the toluene solution of the $\mathrm{Pd}(\mathrm{II})(\mathrm{ac})_{2}$. Oxidation debris does not enter the inner pores of nanotubes, therefore, $\mathrm{Pd}$ nanoparticles anchored on the inner walls of nanotubes are simply not affected by the debris. Nanoparticles anchored on the outer walls of nanotubes seem to be equally insensitive to the presence of oxidation debris. These findings suggest that while post-CCVD impurities affect supported Pd nanoparticle size, oxidation debris does not have significant effect on the nanoparticles.

\subsection{3.e. Catalytic tests}




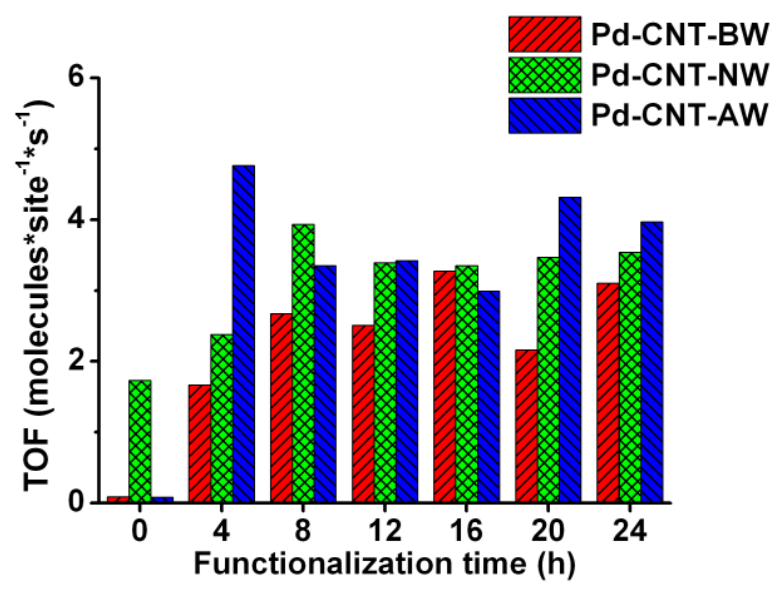

Figure 5.22. The difference in TOF 10 minutes after the initialization of the catalytic reaction. Catalysts supported on non-oxidized CNTs demonstrate the beneficial effect of post-CCVD impurities on conversion. On the other hand, samples oxidized for $4 \mathrm{~h}$ evidence the inhibition effect of oxidation debris as a evidenced by the sudden leap in TOF on "After Washed" sample.

Continuous flow catalytic reaction tests were performed on all samples to reveal further details on the effects of functionalization and washing. The hydrogenation of cyclohexene to cyclohexane was monitored and turnover frequencies (TOF) were calculated for all samples after $10 \mathrm{~min}$ of reaction time (Figure 5.22.).

In case of CNT-NW samples, a TOF value of 1.7 molecules $\cdot \mathrm{site}^{-1} \cdot \mathrm{s}^{-1}$ was measured for the unfunctionalized sample, then increased steadily to 2.4 at $4 \mathrm{~h}$ and reached 3.9 at $8 \mathrm{~h}$ oxidation. No significant changes were observed in the TOF at longer oxidation times.

On the other hand, unfunctionalized Pd-CNT-BW and Pd-CNT-AW samples showed no significant cyclohexene conversion, whereas longer oxidation times resulted in TOF values comparable or even larger than those of the corresponding Pd-CNT-NW samples. This can be attributed to the increasing amount of polar, oxygen containing functional groups on the surface with the progression of functionalization. Interestingly, acetone-washed, nonoxidized $(0 \mathrm{~h})$ samples showed almost negligible TOF values compared to the 1.7 molecules $\cdot \operatorname{site}^{-1} \cdot \mathrm{s}^{-1}$ value of the non-washed one, which seems to indicate that the presence of post-CCVD impurities has a significant effect on the catalytic activity. On the other hand, CNT-AW and CNT-BW samples oxidized for $4 \mathrm{~h}$ exhibited significant hydrogenation activity (1.65 and 4.8 molecules $\cdot \operatorname{sit}^{-1} \cdot \mathrm{s}^{-1}$, respectively) compared to non-oxidized samples. These results show that the presence of the post-CCVD impurities can inhibit the oxidation process and/or the removal of the oxidation debris can enhance catalytic activity.

Nanotubes oxidized for $8 \mathrm{~h}$ or longer show almost identical TOF values for all samples, which suggests that oxidation debris and functional groups do not alter the cyclohexene conversion in these samples as the size of the Pd nanoparticles has not changed 
with oxidation time in the $8-24 \mathrm{~h}$ range. This could be explained by the fact that carbon nanotubes break up into shorter nanotubes during prolonged oxidation [20], thus the shortening of nanotubes reduces transport resistance making the effect of oxidation debris less significant. Moreover, the inner nanotube surfaces are less prone to get covered by oxidation debris because of the narrower entrance diameter of inner nanotube pores compared to intertubular pores of forming CNT agglomerates. This is important, as it is possible for nanoparticles to be anchored in the interior of CNT and therefore, they are less affected by debris. These phenomena can explain why there is no decrease in TOF for CNTNW and CNT-BW samples despite the fact that the specific surface area tends to decrease for functionalization times longer than $8 \mathrm{~h}$. Consequently, oxidation debris blocks mostly intertube pores in the forming agglomerates, but not necessarily the inner pores of tubes.

\subsection{Preparation and characterization of $3 D$ structures based on functionalized carbon nanotubes with no additives.}

In order to provide a means to simply and cost efficiently bring carbon nanotube based materials closer to everyday use, a centrifugation based method has been investigated to form three dimensional structures from carbon nanotubes without the usage of further additives. This method would thus eliminate certain problems associated with composite materials, where the surface of nanotubes and supported metal nanoparticles become mostly inaccessible. Since in industrial catalytic applications powder samples are usually pelleted for better handling and overall throughput, this method could bring carbon nanotubes closer to this kind of application, as the assembly process of such nanotube structures can happen any time after different nanotube modifications.

\subsubsection{Preparation of $3 \mathrm{D}$ structures from carbon nanotubes}

The same MWCNTs were used for creating 3D structures as described in section 5.2 with no additional cleaning (i.e. in Soxhlet extractor). $4 \mathrm{~g}$ of MWCNTs were functionalized for $12 \mathrm{~h}$ by refluxing in $500 \mathrm{~cm}^{3}$ of concentrated nitric acid solution. Nitric acid was removed by centrifugation. CNTs were washed with distilled water until an indication of inadequate deposition of material in the centrifugation tube was evident. The $\mathrm{pH}$ was then adjusted with concentrated $\mathrm{HCl}$ solution to facilitate complete deposition of CNTs and finally the water was decanted to get the butter-like paste material at the bottom of the centrifugation tubes. All centrifugations were conducted at $3200 \mathrm{rpm}$ for $10 \mathrm{~min}$, where the sample rotated at 17 $\mathrm{cm}$ radius suffered a relative centrifuge force of 1950 g. $\sim 10 \mathrm{~g}$ of CNT paste was then 
transferred into a specially manufactured PTFE walled mold (Fig 5.23.), where the perforated bottom part was covered with a nylon membrane $(0.45 \mu \mathrm{m}$ pore size $)$ to support the CNT paste.

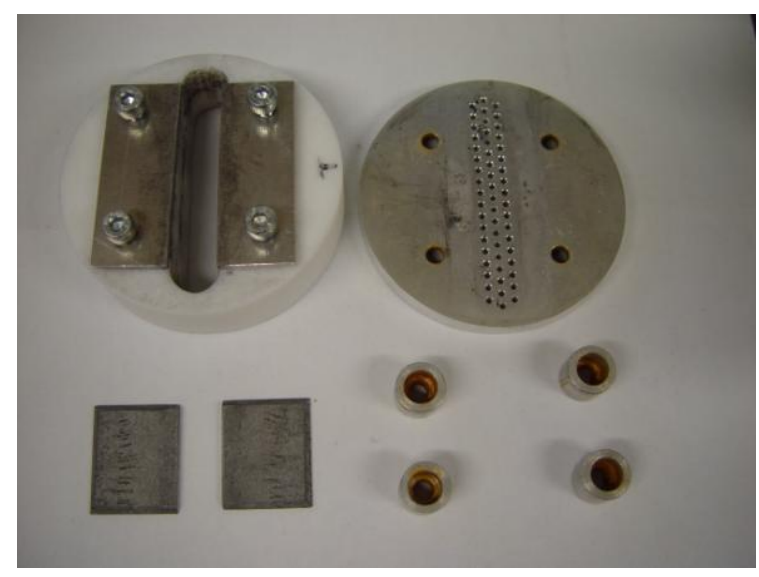

Figure 5.23. The dismountable mold used for creating 3D nanotube structures. The perforation of the bottom aluminum part is covered with nylon membrane to support the CNT paste. The upper PTFE part can be modified with two aluminum plates to create bone-like shapes for tensile testing. The four legs ensure to have enough room for the water to escape to.

Special attention was paid to fill the mold evenly with the paste, as uneven filling resulting in irregularly shaped forms. The CNT filled mold was then centrifuged at $3500 \mathrm{rpm}$ for $10 \mathrm{~min}$ where the sample suffered a relative centrifuge force of $2300 \mathrm{~g}$. As a result of centrifugation 5-6 $\mathrm{g}$ of water evaporated from the sample resulting in a considerable vertical shrinkage of the paste. The as-prepared CNT bar was then sandwiched between two PTFE sheets of $1 \mathrm{~mm}$ thickness and placed into an oven heated to $353 \mathrm{~K}$ for $24 \mathrm{~h}$. The upper PTFE sheet was weighted with 800-1600 g of concrete bricks, depending on the desired final shape of the 3D nanotube structure. Typically, using $800 \mathrm{~g}$ of weight resulted in a boat-like shape, while $1600 \mathrm{~g}$ in a straight CNT bar suitable for mechanical and other types of measurements (Fig 5.24.). The final weight of dried CNT structures was found to be $600 \mathrm{mg}$. 


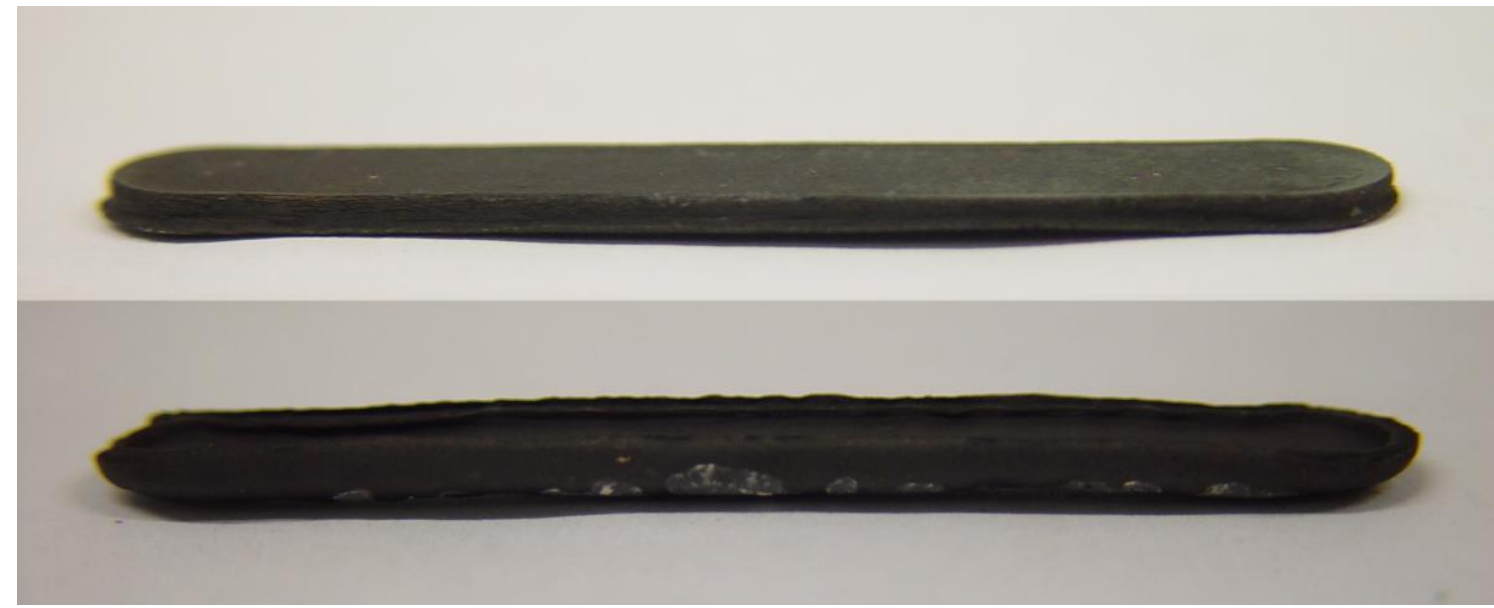

Figure 5.24. A bar shaped (top) and boat shaped (bottom) nanostructure made purely from oxidized carbon nanotubes.

\subsubsection{Characterization of 3D CNT nanostructures}

\subsection{2.a. Scanning electron microscopy}




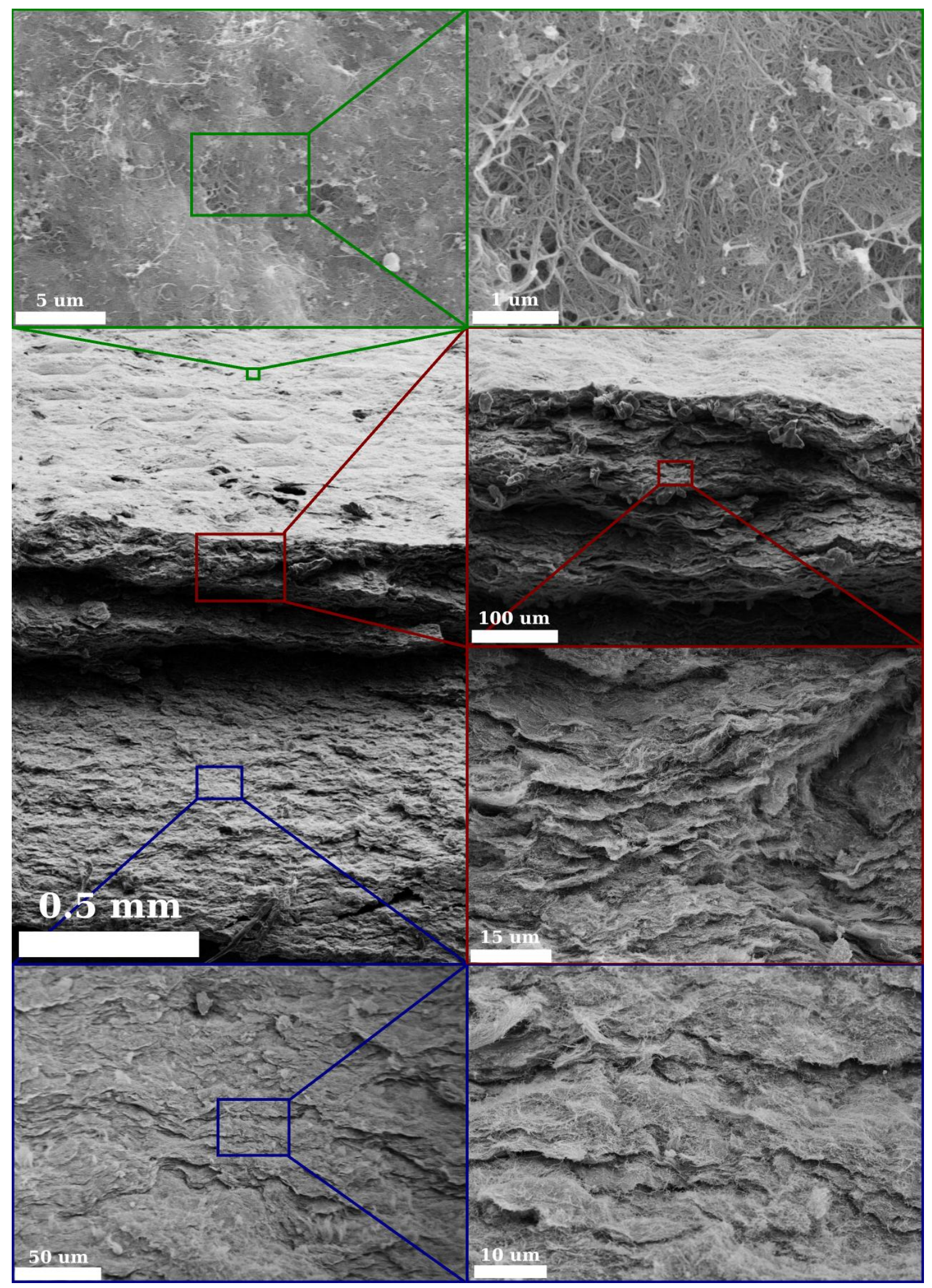

Figure 5.25. SEM micrographs of a CNT bar fracture. Images were taken at the general surface of the bar (green frame) vertical fracture surface (red frame) and low degree fracture surface or facet (blue frame). 
SEM micrographs were captured on a broken CNT bar imaging the resulting new vertical and low angle (facet) breaking surfaces as well as the outer surface. The resulting micrographs are presented in Figure 5.25.

The outer surface of the CNT bar presents a flat surface made up of closely packed individual nanotubes forming a network. Both the vertical breaking surface and facet indicates a non-homogenous inner structure, where nanotubes seem to settle into an interconnected layered structure. This is most probably caused by locally occurring shrinkage of the nanotube network during the drying process, leaving voids between such areas. This assumption is validated by nitrogen adsorption measurements, as discussed in more details later. The overall fracture surface of the nanotube block does not resemble surfaces typical of brittle fracture; instead it shows great resemblance to ductile fracture surfaces of metals caused by tensile tearing.

\subsection{2.b. Nitrogen adsorption measurements}
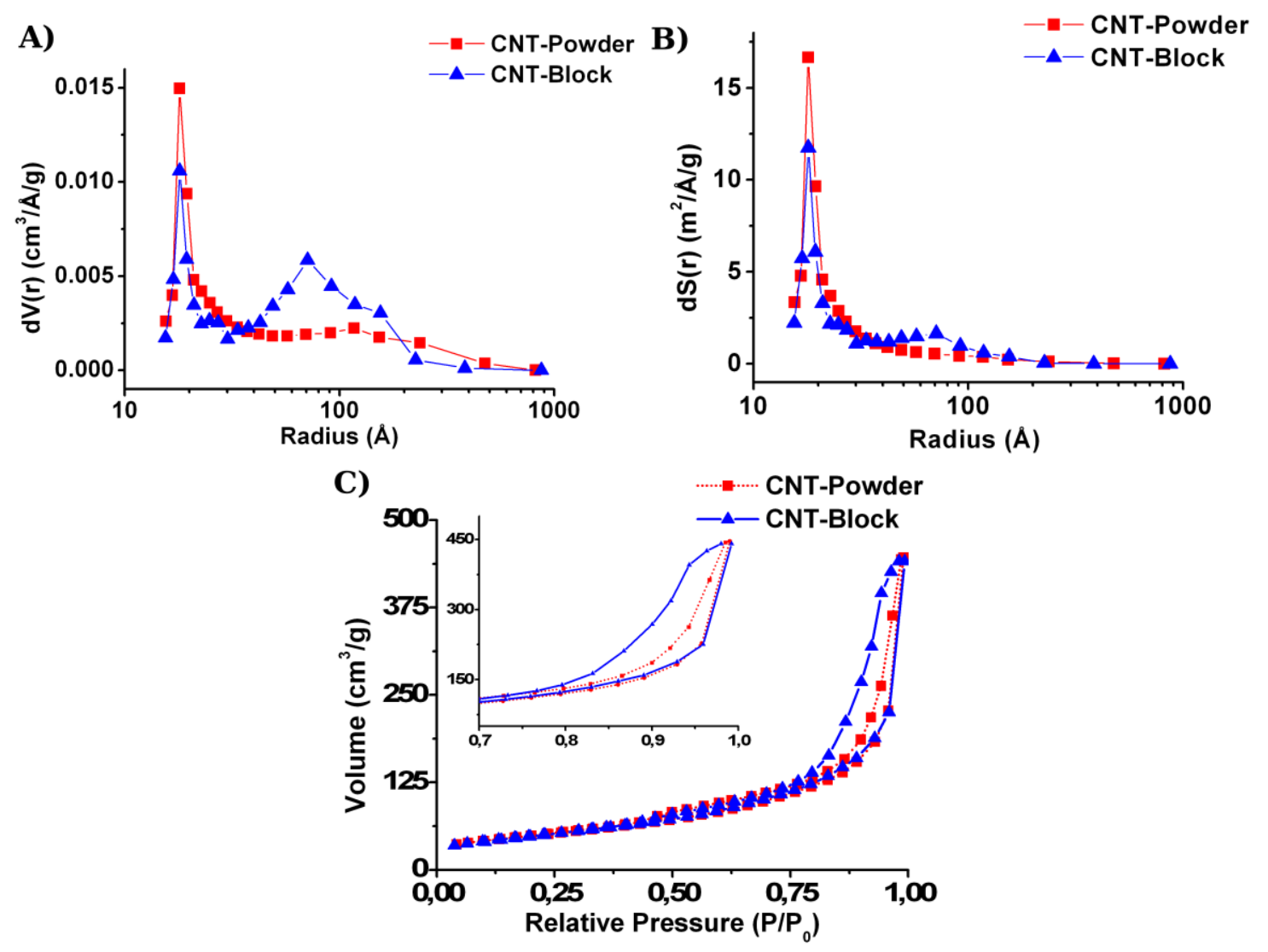

Figure 5.26. Plots of $A$ ) pore size versus volume, B) pore size versus surface area and C) adsorptiondesorption isotherms of CNT samples in powder and 3D block form. 
Nitrogen adsorption measurements were conducted on both powder and 3D structures. Adsorption isotherms and derived pore volume and pore size distribution data plots are presented in Fig. 5.26. Both CNT samples showed typical type IV adsorption curves as classified by IUPAC. The hysteresis curves of the two samples however show notable differences above relative pressure value of 0.75 . While the powder sample exhibits a relatively narrow hysteresis loop, the loop visible for the CNT block sample is considerably wider, which suggest a different pore structure. This is assumption is further validated by pore size distribution data derived from the isotherms as plotted in Fig 5.26 A) and B). All curves show a sharp peak for pore radii of $1.8 \mathrm{~nm}$ which can be considered to be the wellknown TSE artifact. Curves of pore radius versus volume show a gradual decrease in pore radius region 2-4 nm, where the block sample shows slightly lower values. The following 4$20 \mathrm{~nm}$ region then shows a small and larger peak for powder and block samples, respectively, where the values of the block sample are considerably higher. Similar tendencies are visible on pore radius versus surface area plots, with the exception of the CNT powder sample, which does not show a peak in the 4-20 nm region, instead a gradual decrease is visible.

These results mean that the block sample exhibits a slightly smaller amount of pores in the 2-4 $\mathrm{nm}$ region, while considerably more in the $4-20 \mathrm{~nm}$ region. These differences originate from the preparation methods used for both samples. Since powder samples were not centrifuged, CNTs were not forced to pack closest to each other, resulting in a more or less loose network of nanotubes. This pore structure was altered by centrifugation for block samples, resulting in closer packed nanotubes with smaller intertubular pores. The 4-20 nm region on the other hand showed a considerably larger amount of pores for the block sample, which could be the result of both centrifugation and drying process. Nanotubes pack closer during centrifugation, but the packing is restricted by water still present on the surface of nanotubes. It is important to note that not remove all water is removed by centrifugation, a considerable amount ( $\sim 500 \mathrm{wt} \%)$ still remains on the surface and/or intertubular pores of nanotubes. When removed, the nanotube structure shrinks further, creating locally denser areas where nanotubes are packed closest and as a result new voids form between such areas. These newly formed voids are responsible for the appearance of the larger pores in the 4-20 $\mathrm{nm}$ region for centrifuged samples.

Interestingly, calculated specific surface area $\left(173 \mathrm{~m}^{2} / \mathrm{g}\right.$ and $172 \mathrm{~m}^{2} / \mathrm{g}$ for powder and block samples, respectively) was found to be identical for both samples, despite the differences in pore structure. This difference was better represented in calculated total pore volume, where the CNT block exhibited $50 \%$ lower total pore volume $\left(0.69 \mathrm{~cm}^{3} / \mathrm{g}\right.$ and 0.35 
$\mathrm{cm}^{3} / \mathrm{g}$ for powder and block samples, respectively) due to the more dense nature of the structure.

\subsection{2.c. Thermogravimetry}
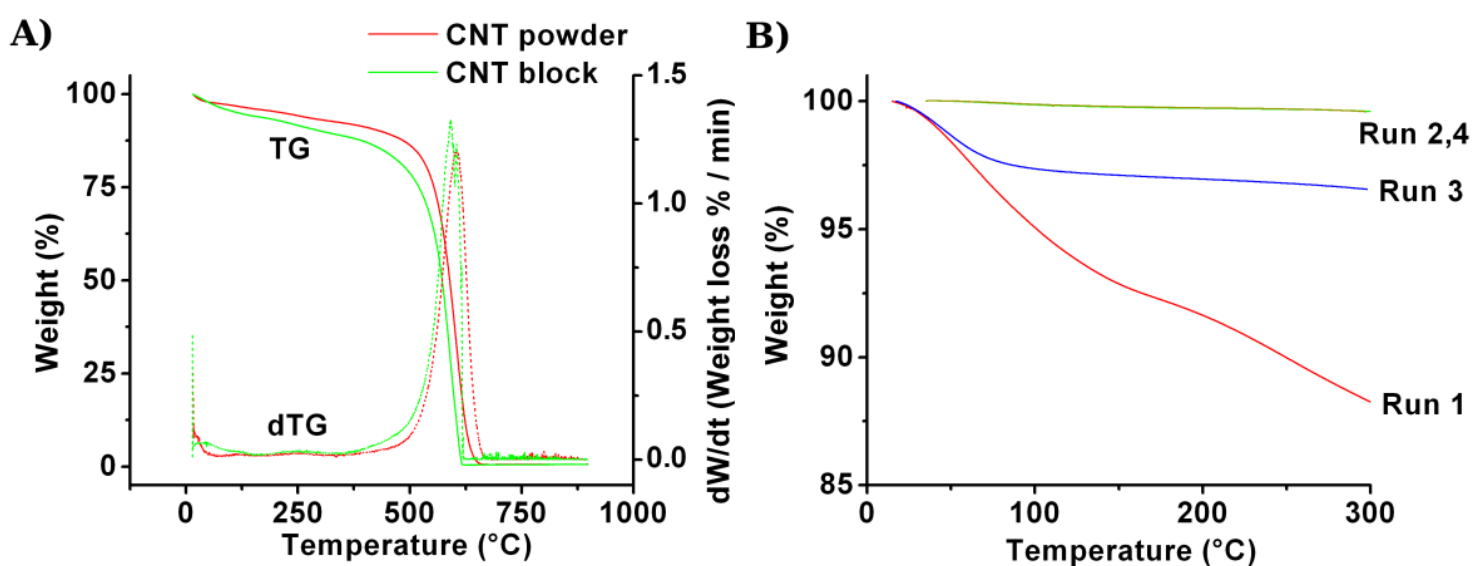

Figure 5.27. A) Thermogravimetric and differential thermogravimetric curves of CNT powder and block samples. B) Repeated thermogravimetric measurements of CNT block samples. Run 2 and Run 4 are measured directly after Run 1 and Run 3, respectively, while Run 3 was conducted after leaving the sample in air at room temperature for $48 \mathrm{~h}$ following Run 2 . Run 3 clearly demonstrates the presence of 2.5 wt\% of water that adsorbed during the 48 hours between Run 2 and Run 3 .

Thermogravimetric measurements were conducted on both powder and block samples to reveal any differences between the thermal stability of powdery and block samples. Thermogravimetric (TG) and differential thermogravimetric (dTG) curves are presented in Fig 5.27.

Both samples show one major and a smaller weight loss, the smaller starting at temperatures as low as $313 \mathrm{~K}$, and lasting up until the major weight loss starts at $648 \mathrm{~K}$. The smaller weight loss can be divided into two parts, beginning with the gradual desorption of water and adsorbed species, and ending with the decomposition of oxygen containing functional groups. The dTG curve shows a small peak starting at $458 \mathrm{~K}$ which most probably corresponds to the starting point for the decomposition of functional groups. Both TG and dTG curves show a slightly higher weight loss for the CNT block in this temperature range, which could correspond to a slightly higher water content of the this sample.

Samples start to burn at $648 \mathrm{~K}$, and the burning process ends at $898 \mathrm{~K}$ and $933 \mathrm{~K}$ for block and powder samples, respectively, where the weight loss is $100 \%$. Interestingly, the block sample seems to burn faster than the powder sample. This suggests that the closer packing of nanotubes helps the burning process by distributing the heat of combustion more efficiently throughout the block, resulting in a slightly higher local temperature around oxidation hot spots. It must be noted though, that the positive effect of heat transportation on 
the oxidation process could be somewhat higher, as a more dense nanotube structure could cause a hindered gas diffusion inside the block, which by itself would lower the burning speed of the nanotubes. This small difference in burning speed thus could be a sum of two concurring processes: the improved heat transportation and the hindered gas diffusion which would increase and decrease the burning speed, respectively.

Repeated heating measurements were also conducted on CNT block samples up until $573 \mathrm{~K}$ (Fig 5.27. B), to reveal water adsorption capabilities. These results are necessary to explain some changes in the mechanical properties of the nanotube block, as explained later. Four consecutive tests were performed, where the first was conducted on a fresh sample and was immediately followed by an identical second one. The third run was conducted after leaving the sample in humid air for $48 \mathrm{~h}$, and was immediately followed by the fourth run. This procedure ensured the precise determination of the amount of species adsorbed from air by eliminating the interference of functional group decomposition.

Run 1 indicates a two-step weight loss in this temperature region, consisting of water desorption and functional group decomposition, as explained earlier. The second run expectedly does not show any noticeable weight changes, as water could not adsorb onto the surface of nanotubes between two runs. Run 3 on the other hand indicates a $2.5 \%$ weight loss up until $373 \mathrm{~K}$, which decrease indicates the amount of water the CNT block adsorbed in the $48 \mathrm{~h}$ between measurements. No further weight loss was observed for Run 3. Expectedly, Run 4 turned out to be identical to Run 2, which again indicates no weight loss whatsoever.

\subsection{2.d. Mechanical properties}

A)

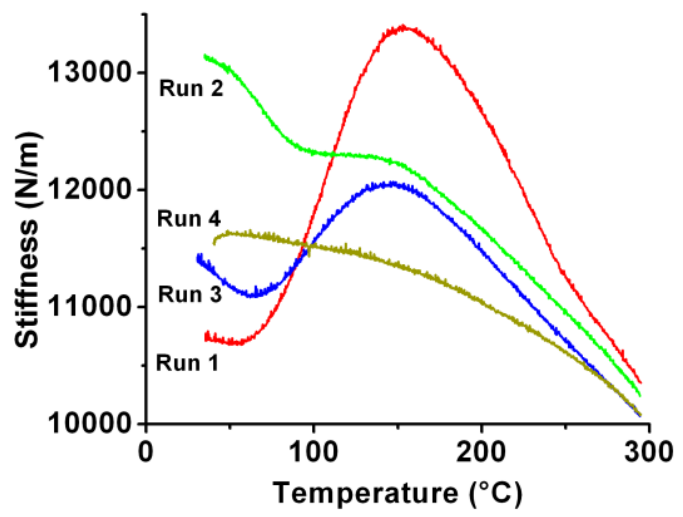

B)

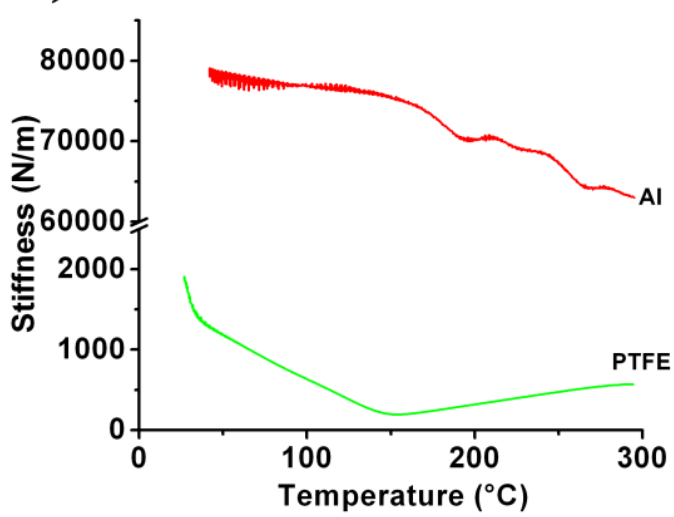

Figure 5.28. Stiffness vs. temperature curves of A) CNT block samples and B) reference materials (aluminum and PTFE) obtained by dinamic mechanical analysis. The mechanical properties of CNT material clearly show a dependence on adsorbed water, as proved by the several consecutive test runs on the same sample. 
Dynamic mechanical analysis and simple elasticity tests were performed to reveal the mechanical properties of the CNT block and to compare these properties with other wellknown materials. Stiffness vs. temperature curves of the CNT material and reference materials are presented in Figure 5.28 A) and B), respectively. Several consecutive measurements were conducted on the CNT block sample, as discussed in the thermogravimetry section. The sample was not removed from the device between test runs to eliminate experimental errors originating from dissimilar mounting of the sample in the cantilever.

Test run 1 was performed on a fresh sample, which exhibited large changes in stiffness in the entire temperature region measured. The stiffness curve starts at $10700 \mathrm{~N} / \mathrm{m}$ which then rises to $13400 \mathrm{~N} / \mathrm{m}$ reaching a maximum at $423 \mathrm{~K}$. The maximum is followed by a steady decrease, which trend does not change in the remaining temperature range of the measurement. To better understand these results, the second measurement (Run 2) was conducted after the sample cooled down to room temperature and the furnace door of the instrument was open. This way moisture found in air could adsorb onto the now activated nanotube surfaces. This resulted in an almost steady decaying curve with a negative peak found between $323 \mathrm{~K}$ and $423 \mathrm{~K}$. This small negative peak must be caused by the small amount of moisture adsorbed during the soaking time between measurements. This means that the initial large positive change for Run 1 in reality is part of a large negative peak caused by large amounts of moisture, as both changes end at $423 \mathrm{~K}$.

To validate this theory, the sample was left for two days in moist air to let proper amounts of water to adsorb, then the CNT block was measured again (Run 3). This resulted in a much a larger negative change at temperatures below $423 \mathrm{~K}$ if compared to Run 2, yet this negative peak still ends exactly at the same temperature as for the first two runs. Run 4 was conducted immediately after Run 3, but the furnace door was not opened this time during sample cooling, thus no significant amounts of water could adsorb onto the nanotubes. This resulted in a steady decaying curve without any negative peaks found, meaning that the cause of this peak really was adsorbed moisture. These results also confirmed by thermogravimetric data, where $2.5 \mathrm{wt} \%$ of water was found to adsorb onto the surface of nanotubes after leaving the samples in moist air for $48 \mathrm{~h}$.

Simple elasticity tests were also performed on both CNT block and an aluminum piece with comparable size. The test consisted of dropping a small steel ball $(\mathrm{d}=5.5 \mathrm{~mm}, \mathrm{~m}=0.68 \mathrm{~g})$ from a predefined height of $265 \mathrm{~mm}$. The relative elasticity of the CNT sample was determined by comparing the height to witch the ball bounced back with an equal sized $\mathrm{Al}$ 
rod. To ensure the repeatability of the ball trajectory, a $105 \mathrm{~mm}$ long glass tube was used to guide the ball during the first $\sim 2 / 3$ part of the fall. The ball then bounced off of the sample and the maximum height the ball achieved was recorded with a high speed camera. Both the aluminum and CNT block samples were fixed onto a $25 \mathrm{~mm}$ thick steel bar with duct tape. Combined images of both experiments are depicted in Fig 5.29.

Balls bounced to heights of $11.8 \mathrm{~cm}$ to $14.3 \mathrm{~cm}$ on aluminum, while $8.9 \mathrm{~cm}$ to $10.4 \mathrm{~cm}$ on CNT block. This could translate into better elastic properties for the aluminum sample, however small indentations were left on the surface of the aluminum after each ball drop. Such marks were not visible for the CNT sample, which means that the deformation induced by the impact of the ball is more elastic in nature for the CNT block, than it is for the aluminum, where a certain amount of plastic deformation is also visible. Interestingly, this completely reversible deformation of the nanotube block absorbs more energy than the elasto-plastic deformation of the aluminum, which could be caused by the friction between individual nanotubes during deformation.

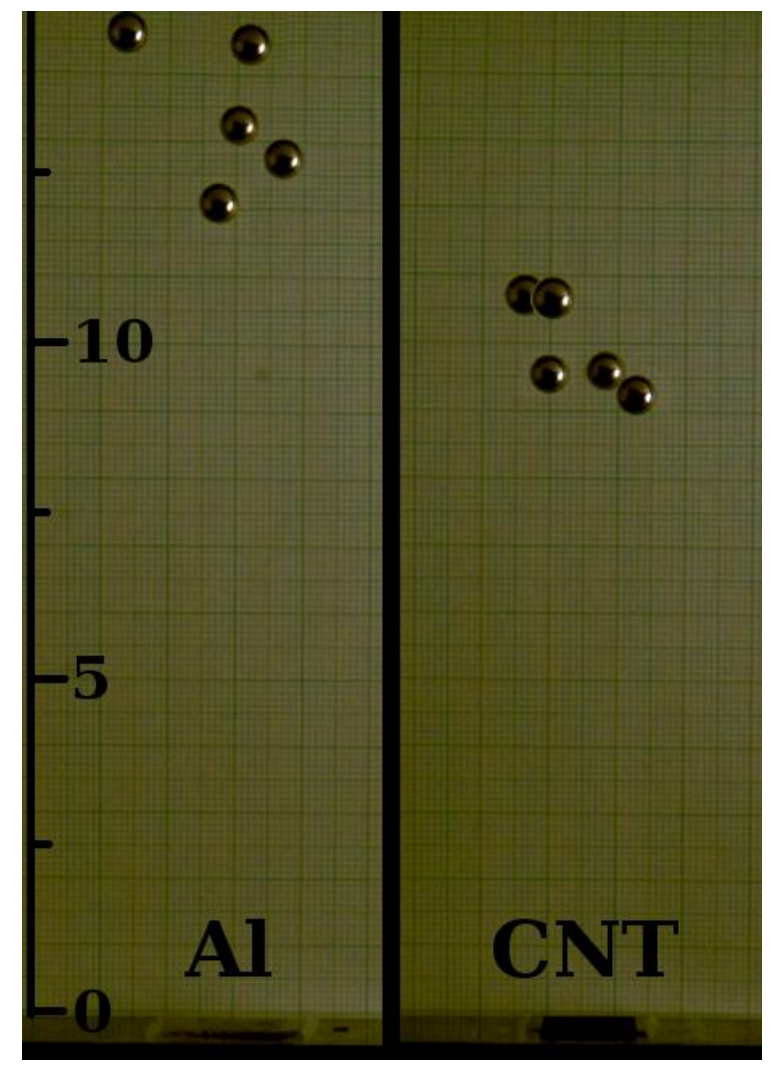

Figure 5.29. A simple elasticity test for aluminum and CNT block samples of comparable size. Steel balls were found to bounce higher on the aluminum sample then on the CNT block, however small indentations were visible on the aluminum sample suggesting elasto-plastic behavior. The CNT bar was free of such marks, yet the balls bounced lower suggesting greater energy absorption. The friction between individual nanotubes during deformation is believed to cause this greater energy absorption. The scale bar is in cm units. 


\section{Summary}

My doctoral research involved the synthesis and characterization of different $\mathrm{Pd}$ decorated carbon structures and 3D nanotube assemblies. The samples were characterized by a wide range of techniques, including electron microscopy, thermogravimetry, spectroscopy, mechanical analysis, nitrogen adsorption, gas chromatography, high speed imaging and others. Sample were prepared in order to reveal more insight into the effect of various surface species like functional groups, amorphous content, oxidation debris, trace elements on particle size, catalytic activity and physical properties of the prepared materials. The possibility of creating 3D nanostructured carbon nanotube assemblies by a simple and convenient method has also been investigated in order to provide better means to transfer the benefits of the wide arrange of nanotube modification methods developed in the past decades into the newly created three dimensional structures.

The main results of this $\mathrm{PhD}$ thesis are the following:

High specific surface area mesoporous carbon samples were successfully synthesized by the hard template method using various commercial Ludox ${ }^{\circledR}$ colloid silicas as template. A simple method was devised for the fine control of total pore volume and specific surface area within a wide range of 0.99 to $4.37 \mathrm{~cm}^{3} / \mathrm{g}$ and 624 to $1630 \mathrm{~m}^{2} / \mathrm{g}$, respectively, by using binary mixtures of the templates. This method provides a much more reliable way to control these properties than other techniques, where the amount of precursors or reaction times are used for this purpose, as in these cases the wall thickness and pore interconnectedness changes between different synthesis setups. Our technique on the other hand is free of such problems, thus provides a more reproducible final product.

Mesoporous carbon materials were successfully modified by Pd using the wet impregnation and "Pd-sol method". The "Pd-sol method" was developed in our laboratory as a one-step method to provide a means for directly investigating the catalytic activity of nanoparticles encapsulated into the carbon structure. The effects of various etching agents and synthesis temperatures were also investigated.

It was found that samples prepared with the "Pd-sol method" showed low TOF values of $\sim 0.5$ molecules $\operatorname{site}^{-1} \cdot \mathrm{s}^{-1}$, meaning that particles encapsulated were still accessible by the reactant. The reactivity of such samples was only modified by carbonization temperature, where lower temperatures resulted in slightly higher TOF which can be attributed to the 
smaller diameter of nanoparticles found on "Pd-sol" modified samples carbonized at lower temperatures.

The effect of specific surface area on catalytic activity was investigated by changing this value through carbonization temperature and etching method. Higher carbonization temperatures and HF etching both provided higher specific surface areas. In case of HF etched samples made by the impregnation method, higher carbonization temperature resulted in higher $S_{\mathrm{BET}}$ which resulted in a twice as high TOF compared to samples carbonized at a lower temperature. Specific surface area however does not always provide clear advantage, as samples containing minute amounts of sodium, which are known to promote certain catalytic reactions, showed much higher TOF, even though the specific surface area of such samples were considerably lower. These results show that higher specific surface area is only beneficial in certain cases only.

The effect of carbonization temperature and etching method on nanoparticle size was also investigated. If nanoparticles were deposited prior to synthesis, a direct correlation was found between nanoparticle size and carbonization temperature, however nanoparticles deposited after carbon synthesis were not sensitive to carbonization temperature. No direct correlation was found between etching method and nanoparticle size. These results showed that the general surface properties of the samples prepared by various carbonization temperatures and etching methods are similar, as nanoparticle size is sensitive to differences in surface environment.

Carbon nanotubes were oxidized and modified with Pd nanoparticles to reveal the exact role of post-synthetic impurities, oxidation debris and functional groups on the surface of nanotubes. For this purpose several sample series were created involving different functionalization times and Soxhlet-extractor enhanced acetone washing. Prepared samples were also subjected to catalytic tests to reveal further information, as such reactions are sensitive to changes in surface environment.

Oxidation time was found to affect the diameter of supported nanoparticles, regardless of the nanoparticles being metallic or oxide. The change of particle diameters closely followed Raman G/D ratios. This means that even though oxide and metallic nanoparticles are electronically different, the surface environment affects the formation of both types of nanoparticles identically. Evidence was also found that nanoparticles initially formed on the surface of nanotubes are made up of several smaller crystallites, which then sinter at moderately high temperatures to form a single crystal nanoparticle. This was found to be 
significant as the sintering temperatures employed were identical to the temperatures where the activation of the catalysts usually happens, thus this post-synthetic transformation of catalysts must carefully be taken into account at all characterization attempts of nanotube supported.

Incorporating a Soxhlet-extractor enhanced acetone washing into the synthesis procedure allowed the creation of a series of oxidized nanotubes, where the effects of oxidation debris, functional groups and post-synthetic impurities could separately be studied. It has been found that post-synthetic impurities negatively affected specific surface area of samples as well as particle diameters. In the absence of such impurities nanoparticles exhibited noticeably smaller diameters, while specific surface area was found to be higher. Surprisingly, cyclohexene hydrogenation was positively affected by the presence of such impurities, as non functionalized nanotubes showed considerably higher TOF then non functionalized samples freed from such moieties.

Oxidation debris on the other hand had little effect on the hydrogenation reaction, while specific surface area was negatively affected by these moieties. The negative effect on $\mathrm{S}_{\mathrm{BET}}$ was found to be caused by the blockage of intertubular pores. This blockage however had little effect on the diffusion of the hydrocarbons during catalytic tests, mostly because of the shortening of nanotubes caused by prolonged oxidation, which in turn significantly decreased transport resistance. The shortening of nanotubes is especially beneficial for particles residing in the inner surface of nanotubes, as these particles are the most affected by diffusion limitation phenomena. The presence of oxidation debris was also found not to have any significant effect on supported nanoparticle size.

Surface functional groups were not found to affect specific surface area or nanoparticle size contrary to statements found many times in literature. Functional groups on the other hand seem to have a beneficial effect on the catalytic reaction, which is caused by changed sorption capabilities of hydrocarbons.

A new, simple method was successfully devised for the creation of 3D nanotube assemblies made purely of carbon nanotubes. This method allows the utilization of various functionalized or decorated carbon nanotubes, as the assembly of nanotubes can happen any time after various treatments. Also, no high temperature treatments are involved in the assembly process, thus no degradation is expected even from sensitive surface functionalities as biomolecules. The physical properties of as prepared assemblies were found to be affected by the amount of adsorbed water. Dried materials were capable of taking up water from 
moist air up to $2.5 \mathrm{wt} \%$. Signs of material fatigue were also found after repeated measurements. Simple elasticity tests showed greater energy absorption from sample deformation if compared to aluminum, which might be caused by friction between individual nanotubes during deformation. 


\section{Acknowledgement}

First of all I wish to thank my family for their complete confidence and love during my graduate studies.

I am heartily thankful to my supervisor Prof. Zoltán Kónya for guiding my work and for his useful suggestions regarding my work. I also thank Prof. Ákos Kukovecz for all the additional insights and discussions related to my work. I would also like to thank both of them for providing all the means for doing my research and all the opportunities to learn new things throughout the years. Without their help and support none of this would have been possible.

I would also like to thank Prof. Imre Kiricsi, the late head of Department of Applied and Environmental Chemistry, who first made it possible for me to work at the department as a young student almost a decade ago.

I would also like to thank my colleague Dr. András Sápi for his many encouraging words, useful suggestions, and generally the huge amount of help he provided me over the years.

I am also grateful for the help and patience of my colleague Dr. Erzsébet Bogya, who contributed a lot to my work in the short amount of time I worked with her.

I also thank all the present and past staff of the Department of Applied and Environmental Chemistry of University of Szeged for their patience, support and sense of humor.

I also express my gratitude to Prof. Krisztián Kordás at University of Oulu for showing great understanding during the writing of my thesis.

I also thank all the people outside the Department who contributed in some way to my work with either suggestions, advice, comments or otherwise.

This research was realized in the frames of TÁMOP 4.2.4. A/2-11-1-2012-0001 „National Excellence Program - Elaborating and operating an inland student and researcher personal support system convergence program" The project was subsidized by the European Union and co-financed by the European Social Fund. 


\section{References}

1 H. Jager, W. Frohs, G. Collin, F. von Sturm, O. Vohler, G. Nutsch; Ullmann's Encyclopedia of Industrial Chemistry DOI: 10.1002/14356007.a05_095.pub2

2 J.A. Menendez-Diaz, I. Martin-Gullon; T.J. Bandosz, eds., Activated Carbon Surfaces in Environmental Remediation, Elsevier, 2006, pp. 1-47

${ }^{3}$ A. Dabrowski; Adv. Colloid Interface Sci. 93 (2001) 135-224

${ }^{4}$ V.J. Inglezakis, S.G. Poulopoulos, Adsorption, Ion Exchange and Catalysis: Design of Operations and Environmental Applications, Elsevier 2006

${ }^{5}$ University of Kentucky, Center for Applied Energy Research, web information. Available at www.caer.uky.edu/carbon/history/carbonhistory.shtml., Accessed: August, 2015

${ }^{6}$ F. Cecen, O. Aktas, Activated Carbon for Water and Wastewater Treatment: Integration of Adsorption and Biological Treatment, Wiley-VCH, 2011

7 Boardman, John. "The Neolithic-Eneolithic Period". The Cambridge ancient history, Volume 3, Part 1 pp. 31-32. ISBN 0521224969

${ }^{8}$ U.S. Geological Survey, 2005, graphite statistics, in Kelly, T.D., and Matos, G.R., comps., Historical statistics for mineral and material commodities in the United States: U.S. Geological Survey Data Series 140, available online at http://pubs.usgs.gov/ds/2005/140/ (Accessed: August 2015.)

${ }^{9}$ U.S. Geological Survey, 2005, diamond statistics, in Kelly, T.D., and Matos, G.R., comps., Historical statistics for mineral and material commodities in the United States: U.S. Geological Survey Data Series 140, available online at http://pubs.usgs.gov/ds/2005/140/ (Accessed: August 2015.)

${ }^{10}$ G. Lalwani,B. Sitharaman; Nano Life. 3 (2013) 1342003

${ }^{11}$ J.H. Knox, K.K. Unger, H. Mueller; J. Liq. Chromatogr. 1 (1983) 6

12 J.H. Knox, B.Kaur, G.R. Millward; J. Chromatogr. 3 (1986) 352

${ }^{13}$ D. Kawashima, T. Aihara, Y. Kobayashi, T. Kyotani, A. Tomita,; Chem. Mater. 12 (2000) 3397

${ }^{14}$ T. Kyotani, L.F. Tsai, A. Tomita; Chem. Mater. 8 (1996) 2109

${ }^{15}$ T. Kyotani, L.F. Tsai, A. Tomita; Chem. Mater. 7 (1995) 1427

16 T.F. Baumann, J.H. Satcher; Chem. Mater. 15 (2003) 37

${ }^{17}$ J. Lee, K. Sohn, T. Hyeon; J. Am. Chem. Soc. 123 (2001) 5146.

${ }^{18}$ A.B. Fuertes; Chem Mater 16 (2004) 449

${ }^{19}$ Y. Oda, K. Fukuyama, K. Nishikawa, S. Namba, H. Yoshitake, T. Tatsumi; Chem. Mater. 16 (2004) 3860

${ }^{20}$ S.J. Han, T. Hyeon; Chem. Commun. 19 (1999) 1955

${ }^{21}$ S.J. Han, K. Sohn, T. Hyeon; Chem. Mater. 12 (2000) 3337

${ }^{22}$ S.B. Yoon, G.S. Chai, S.K. Kang, J.S. Yu, K.P. Gierszal, M. Jaroniec; J. Am. Chem. Soc. 127 (2005) 4188

${ }^{23}$ J. Lee, J. Kim, T. Hyeon; Chem. Commun. 10 (2003) 1138

${ }^{24}$ K.P. Gierszal, M. Jaroniec; Chem. Commun. 22 (2004) 2576.

25 A.A. Zakhidov, R.H. Baughman, Z. Iqbal, C.X. Cui, I. Khayrullin, S.O. Dantas, et al; Science 282 (1998) 897

${ }^{26}$ R. Ryoo, S.H. Joo, S. Jun; J. Phys. Chem. B 103 (1999) 7743.

${ }^{27}$ M. Kaneda, T. Tsubakiyama, A. Carlsson, Y. Sakamoto, T. Ohsuna, O. Terasaki, et al; J. Phys. Chem. B 106 (2002) 1256.

28 J. Lee, S. Yoon, S.M. Oh, C.H. Shin, T. Hyeon; Adv. Mater. 12 (2000) 359

${ }^{29}$ B.Z. Tian, S.N. Che, Z. Liu, X.Y. Liu, W.B. Fan, T. Tatsumi, et al; Chem. Commun. 21 (2003) 2726

${ }^{30}$ J. Ren, J. Ding, K-Y. Chan, H. Wang; Chem. Mater. 19 (2007) 2786 
31 J. Parmentier, C. Vix-Guterl, P. Gibot, M. Reda, M. Ilescu, J. Werckmann et al; Microporous Mesoporous Mater. 62 (2003) 87

32 A.Y. Lo, S.J. Huang, W.H. Chen, Y.R. Peng, C.T. Kuo, S.B. Liu; Thin Solid Films 498 (2006) 193

${ }^{33}$ Y. Xia, Z. Yang, R. Mokaya; J. Phys. Chem. B 108 (2004) 19293

${ }^{34}$ Y. Xia, R. Mokaya; Adv. Mater. 16 (2004) 886

${ }^{35}$ R. Ryoo, S.H. Joo, M. Kruk, M. Jaroniec; Adv. Mater. 13 (2001) 677.

${ }^{36}$ S.H. Joo, S.J. Choi, I. Oh, J. Kwak, Z. Liu, O. Terasaki, R. Ryoo; Nature 412 (2001) 169.

${ }^{37}$ M. Kruk, M. Jaroniec, T.W. Kim, R. Ryoo; Chem. Mater. 15 (2003) 2815.

${ }^{38}$ A.A. Attia, W.E. Rashwan, S.A. Khedr; Dyes Pigment 69 (2006) 128-136

${ }^{39}$ P. Chingombe, B. Saha, R.J. Wakeman; J. Colloid Interface Sci 297 (2006) 434

${ }^{40}$ J.R. Rangal-Mendez, F.S. Cannon; Carbon 43 (2005) 467

${ }^{41}$ H.L. Chiang, P.C. Chiang, C.P. Huang; Chemosphere 47 (2002) 267

${ }^{42}$ D. Aggarwal, M. Goyal, R.C. Bansal; Carbon 37 (1999) 1989

${ }^{43}$ M.M. Marato-Valer, I. Dranca, T. Lupascu, R. Nastas; Carbon 42 (2004) 2655

${ }^{44}$ A. Aburub, D.E. Wurster, J. Colloid Interface Sci. 296 (2006) 79

45 A.B. Garcia, A. Martinez-Alonso, C.A.L.Y. Leon, J.M.D. Tascon; Fuel 77 (1998) 613

${ }^{46}$ M. Domingo-Garcia, F.J. Lopez-Garzon, M. Perez-Mendoza; J. Colloid Interface Sci. 222 (2000) 233

${ }^{47}$ H.L. Chiang, C.P. Huang, P.C. Chiang; Chemosphere 47 (2002) 257-265

${ }^{48}$ J. Przepiorski. J. Hazard. Mater 135 (2006) 453

${ }^{49}$ W. Chen, F.S. Cannon, J.R. Rangel-Mendez; Carbon 43 (2005) 573

${ }^{50}$ W. Chen, F.S. Cannon, J.R. Rangel-Mendez; Carbon 43 (2005) 581

${ }^{51}$ N. Adhoum, L. Monser; Sep. Purif. Technol. 38 (2004) 233

${ }^{52}$ C.P. Huang, L.M. Vane, J. Water Pollut. Contam. Fed. 61 (1989) 1596

${ }^{53}$ R. Leyva Ramos, J. Ovalle-Turrubiartes, M.A. Sanchez-Castillo; Carbon 37 (1999) 609

${ }^{54}$ X, Wang, Q. Li, J. Xie, Z. Jin, J. Wang, Y. Li et al; Nano Lett. 9 (2009) 3137

${ }^{55}$ R. Jasti, J. Bhattacharjee, J.B. Neaton and C.R. Bertozzi; J. Am. Chem. Soc, 130 (2008) 17646.

${ }^{56}$ N. Hamada, S.I. Sawada and A. Oshiyama; Phys. Rev. Lett., 68 (1992) 1579

${ }^{57}$ S. Iijima; Nature, 354 (1991) 56

${ }^{58}$ A. Leonhardt, M. Ritschel, K. Bartsch, A. Graff, C. Taschner, J. Fink; J. Phys. IV: Proc., 11 (2001) 445

59 Y.Y. Wang, G.Y. Tang, F.A.M. Koeck, B. Brown, J.M. Garguilo, R.J. Nemanich; Diamond Relat. Mater. 13 (2004) 1287

${ }^{60}$ Y. Saito, M. Okuda, N. Fujimoto, T. Yoshikawa, M. Tomita, T. Hayashi; Jpn. J. Appl. Phys. 33 (1994) L526.

${ }^{61}$ S.J. Lee, H.K. Baik, J. Yoo, J.H. Han; Diam. Rel. Mat. 11 (2002) 914

62 J. Journet, W.K. Maser, P. Bernier,A. Loiseau, M.L. de la Chapelle, S. Lefrant, et al; Nature 388 (1997) 756

${ }^{63}$ M. Yao, B. Liu, Y. Zou, L. Wang, D. Li, T. Cui, et al; Carbon 43 (2005) 2894

64 J.L. Hutchison, N.A. Kiselev, E.P. Krinichnaya, A.V. Krestinin, R.O. Loutfy, A.P. Morawsky; Carbon 39 (2001) 761

${ }^{65}$ Y. Saito, T. Nakahira, S. Uemura;. J. Phys. Chem. B 107 (2003) 931

${ }^{66}$ H. Huang, H. Kajiura, S. Tsutsui, Y Murakami, M. Ata; J. Phys. Chem. B 107 (2003) 8794

${ }^{67}$ Y.-H .Wang, S.-Ch .Chiu, K.-M. Lin, Y.-Y. Li; Carbon 42 (2004) 2535

${ }^{68}$ M. Ishigami, J. Cumings, A. Zettl, S. Chen;. Chem. Phys. Lett. 319 (2000) 457

${ }^{69}$ N. Sano, J. Nakano, T. Kanki; Carbon 42 (2004) 686

${ }^{70}$ M.V. Antisari, R. Marazzi, R. Krsmanovic; Carbon 41 (2003) 2393 
${ }^{71}$ S.-D.Wang, M.-H. Chang, K.M.-D. Lan, Ch-Ch. Wu, J.-J. Cheng, H.-K. Chang; Carbon 43 (2005) 1778

72 T. Guo, P. Nikolaev, A.G. Rinzler, D. Tomanek, D.T. Colbert, R.E. Smalley; J. Phys. Chem. 99 (1995) 10694

${ }^{73}$ M. Yudasaka, R. Yamada, N. Sensui, T. Wilkins, T. Ichihashi, S. Iijima; J. Phys. Chem. B 103 (1999) 6224

${ }^{74}$ M. Zhang, M. Yudasaka, S. Iijima; Chem. Phys. Lett. 336 (2001) 196

${ }^{75}$ H. Kataura, Y. Kumazawa, Y. Maniwa, Y. Ohtsuka, R. Sen, S. Suzuki, et al; Carbon 38 (2000) 1691

${ }^{76}$ D. Nishide, H. Kataura, S. Suzuki, K. Tsukagoshi, Y. Aoyagi, Y. Achiba; Chem. Phys. Lett. 372 (2003) 45

${ }^{77}$ M. Yudasaka, T. Komatsu, T. Ichihashi, Y. Achiba, S. Iijima; J. Phys. Chem. B 102 (1998) 4892

${ }^{78}$ A.A. Gorbunov, R. Friedlein, O. Jost, M.S. Golden, J. Fink, W. Pompe; Appl. Phys. A 69 (1999) S593

${ }^{79}$ N. Braidy, M.A. El Khakani, G.A. Botton; Carbon 40 (2002) 2835

${ }^{80}$ F. Kokai, K. Takahashi, M. Yudasaka, R. Yamada, T. Ichihashi, S. Iijima; J. Phys. Chem. B 103 (1999) 4346

${ }^{81}$ S. Bandow, S. Asaka, Y.A.M. Rao, L. Grigorian, E. Richter, P.C. Eklund, Phys. Rev. Lett. 80 (1998) 3779

${ }^{82}$ A.C. Dillon, P.A. Parilla, J.L. Alleman, J.D. Perkins, M.J. Heben; Chem. Phys. Lett. 316 (2000) 13

${ }^{83}$ M. Yudasaka, T. Ichihashi, T. Komatsu, S. Iijima; Chem. Phys. Lett. 299 (1999) 91

${ }^{84}$ H. Zhang, Y. Ding, Ch. Wu, Y. Chen, Y. Zhu, Y. He et al; Physica B 325 (2003) 224

${ }^{85}$ P. Ahmad, M.U. Khandaker, Z.R. Khan, Y.M. Amin; RSC Adv. 5 (2015) 35116

${ }^{86}$ L.V. Radushkevich, V.M. Lukyanovich; Soviet J. Chem. Phys. 26 (1952) 88

${ }^{87}$ A. Oberlin, M. Endo, T. Koyama; J. Cryst. Growth 32 (1976) 335

${ }^{88}$ S. Iijima; Nature. 354 (1991) 56

${ }^{89}$ M.J. Yacaman, M.M. Yoshida, L. Rendon, J.G. Santiesteban; Appl. Phys. Lett.62 (1993) 202

${ }^{90}$ D. Golberg, Y. Bando, C. Tang, C. Zhi; 3rd International Nanoelectronics conference (INEC) IEEE, (2010) 47

${ }^{91}$ S. Mori, M. Suzuki; Thin Solid Films 517 (2009) 4264

${ }_{92}$ A.H. Jayatissa, K. Guo; Vacuum 83 (2009) 853

${ }^{93}$ K. Hata, D.H. Futaba, K. Nizuno, T. Namai, M. Yumura, S. Iijima; Science 306 (2004) 1362

${ }^{94}$ S. Chaisitsak, J. Nukeaw, A. Tuantramont; Diamond Rel. Mat. 16 (2007) 1958

${ }^{95}$ C. Oncel, Y. Yurum;. Fuller. Nanotub. Car. N. 14 (2006) 17

${ }^{96}$ A.K. Sinha, D.W. Hwang, L.-P. Hwang; Chem. Phys. Lett. 332 (2000) 455

${ }^{97}$ K. Mukhopadhyay, A. Koshio, T. Sugai, N. Tanaka, H. Shinohara, Z. Konya, et al; Chem. Phys. Lett. 303 (1999) 117

${ }^{98}$ S. Zhu, C.-H. Su, S.L. Lehoczky, I. Muntele, D. Ila; Diam. Rel. Mat. 12 (2003) 1825.

${ }^{99}$ Z. Jia, Z. Wang, J. Liang, B. Wei, D. Wu; Carbon 37 (1999) 903.

${ }^{100}$ D.C. Lee, F.V. Mikulec, B.A. Korgel. J. Am. Chem. Soc., 126 (2004) 4951

${ }^{101}$ M. Maynea, N. Grobert, M. Terrones, R. Kamalakaran, M. Rühle, H.W. Kroto, et al; Chem. Phys. Lett. 338 (2001) 101

${ }_{102}$ W. Wasel, K. Kuwana, K. Saito; Chem. Phys. Lett. 422 (2006) 470

${ }^{103}$ G. Ortega-Cervantez, G. Rueda-Morales, J. Ortiz-Lopez; Microelectron. J. 36 (2005) 495

${ }^{104}$ T. Okazaki, H. Shinohara; Chem. Phys. Lett. 376 (2003) 606 
${ }^{105}$ K. Hernadi, A. Fonseca, J.B Nagy, A. Siska, I. Kiricsi. Appl. Catal. A. Gen. 199 (2000) 245

${ }^{106}$ J. Liu, M. Shao, X. Chen, W. Yu, X. Liu, Y. Qian. J. Am. Chem. Soc. 125 (2003) 8088

107 J.T. McCann, B. Lim, R. Ostermann, M. Rycenga, M. Marquez, Y. Xia, Nano Lett. 7 (2007) 2470

${ }^{108}$ M. Laskoski, T.M. Keller, S.B. Qadri; Polymer 48 (2007) 7484

${ }^{109}$ L.C. Yang, Y. Shi, Q.S. Gao, B. Wang, Y.P. Wu, Y. Tang; Carbon 46 (2008) 1816

${ }^{110}$ S. Shang, X. Yang, X. Tao; Polymer 50 (2009) 2815

111 C.M. Yang, C. Weidemthaler, B. Spliethoff, M. Mayanna, F. Schuth; Chem. Mat. 17 (2005) 355

112 J. Jang, H. Yoon; Adv. Mater. 15 (2003) 2088

113 A. Kukovecz, Z. Konya, N. Nagaraju, I. Willems, A. Tamasi, A. Fonseca et al; Phys. Chem. Chem. Phys. 2 (2000) 3071

114 M.A. Ermakova, D.Y. Ermakov, A.L. Chuvilin, G.G. Kuvshinov; J. Catal. 201 (2001) 183

${ }^{115}$ S.S. Pan, Z.W. Xie, B.H. Chang, L.F. Sun, W.Y. Zhou, G. Wang; Chem. Phys. Lett.; 299 (1999) 97

${ }_{116}^{116}$ D. Venegoni, P. Serp, R. Feurer, Y. Kihn, C. Vahlas, P. Kalck; Carbon 40 (2002) 1799

${ }^{117}$ H. Ago, T. Komatsu, S. Ohshima, Y. Kuriki, M. Yumura; Appl. Phys. Lett. 77 (2000) 79.

${ }^{118}$ C.M. Hsu, H.L. Lin, C.H. Chang, C.T. Kuo; Thin solid films 420-421 (2002) 225

${ }^{119}$ C. Klinke, J.-M. Bonard, K. Kern;. Surf. Sci. 492 (2001) 195

${ }^{120}$ M. Yudasaka, R. Kikuchi, T. Matsui, O. Yoshimasa, S. Yoshimura; Appl. Phys. Lett. 67 (1995) 2477

${ }^{121}$ K.B.K. Teo, M. Chhowalla, G.A.J. Amaratunga, W.I. Milne, D.G. Hasko, G. Pirio et al; Appl. Phys. Lett. 79 (2001) 1534.

${ }^{122}$ Z.F. Ren, Z.P. Huang, J.W. Xu, J.H. Wang, P. Bush, M.P. Siegal et al; Science;282 (1998) 1105

${ }^{123}$ H.M. Cheng, S.F. Li, G. Pan, H.Y. Pan, L.L. He, X. Sun et al; Appl. Phys. Lett. 72 (1998) 3282

${ }^{124}$ Y. Li, W. Kim, Y. Zhang, M. Rolandi, D. Wang, H. Dai; J. Phys. Chem. B 105 (2001) 11424

${ }^{125}$ C.L. Cheung, A. Kurtz, H. Park, C.M. Lieber; J. Phys. Chem. B 106 (2002) 2429

${ }^{126}$ P. Nikolaev, M. Bronikowski, R. Bradley, F. Rohmund, D. Colbert, K. Smith et al; Chem. Phys. Lett. 313 (1999) 91

${ }^{127}$ J.F. Colomer, J.F. Bister, I. Willems, Z. Konya, A. Fonseca, G. Van Tendeloo et al; Chem. Commun.;14 (1999) 1343

${ }^{128}$ B.C. Satishkumar, A. Govindaraj, R. Sen, C.N.R. Rao; Chem. Phys. Lett. 293 (1998) 47

129 J.-F. Colomer, C. Stephan, S. Lefrant, G. Van Tendeloo, I. Willems, Z. Konya et al; Chem. Phys. Lett. 317 (2000) 83

${ }^{130}$ R. Seidel, G.S. Duesberg, E. Unger, A.P. Graham, M. Liebau, F. Kreupl; J. Phys. Chem. $B ; \mathbf{1 0 8}(2004) 1888$

${ }^{131}$ Y. Yang, Z. Hu, Y.J. Tian, Y.N. Lu, X.Z. Wang, Y. Chen; Nanotechnology;14 (2003) 733

132 A.M. Cassel, G.C. McCool, H. Tee Ng, J.E. Koehne, B. Chen, J. Li et al; Appl. Phys. Lett. 82 (2003) 817

${ }^{133}$ B. Chen, G. Parker, J. Han, M. Meyyappan, A.M. Cassell; Chem. Mater. 14 (2002) 1891

${ }^{134}$ I. Willems, Z. Konya, J.-F. Colomer, G. Van Tendeloo, N. Nagaraju, A. Fonseca et al; Chem. Phys. Lett. 317 (2000) 71

${ }^{135}$ R. Vajtai, K. Kordas, B.Q. Wei, J. Bekesi, S. Leppavuori, T.F. George et al; Mater. Sci. Eng. C; 19 (2002) 271 
${ }^{136}$ H. Cui, G. Eres, J.Y. Howe, A. Puretkzy, M. Varela, D.B. Geohegan et al; Chem. Phys. Lett.;374 (2003) 222

${ }^{137}$ M. Su, B. Zheng, J. Liu; Chem. Phys. Lett. 322 (2000) 321

${ }^{138}$ H. Ago, S. Ohshima, K. Uchida, M. Yumura; J. Phys. Chem. B; 105 (2001) 10453

${ }^{139}$ W. Alvarez, B. Kitiyanan, A. Borgna, D. Resasco. Carbon; 39 (2001) 547

140 B. Kitiyanan, W.E. Alvarez, J.H. Harwell, D.E. Resasco; Chem. Phys. Lett. 317 (2000) 497

${ }^{141}$ C.J. Lee, J. Park, J.M. Kim, Y. Huh, J.Y. Lee, K.S. No; Chem. Phys. Lett. 327 (2000) 277

${ }^{142}$ N. Halonen, A. Sapi, L. Nagy, R. Puskas, A.R. Leino, J. Maklin et al; Phys. Status Solidi B 248 (2011) 2500

${ }^{143}$ Y. Gogotsi, J.A. Libera, M. Yoshimura; J. Mat. Res. 15 (2000) 2591

${ }^{144}$ S. Manafi; H. Nadali; H.R. Irani; Mat. Lett. 62 (2008) 4175

${ }^{145}$ W.K. Hsu, J.P. Hare, M. Terrones, H.W. Kroto, D.R.M. Walton, P.J.H. Harris; Nature, 377 (1995) 687

${ }^{146}$ G.Z. Chen, X. Fan, A. Luget, M.S.P. Shaffer, D.J. Fray, A.H. Windle; J. Electroanal. Chem. 446 (1998) 1

${ }^{147}$ M.R.A. Leela, S. Ramaprabhu, Int. J. Hydrogen Energy 32 (2007) 3998

148 C.E. Hong, J.H. Lee, P. Kalappa, S.G. Advani; Compos. Sci. Technol. 67 (2007) 1027

${ }^{149}$ I.D. Rosca, F. Watari, M. Uo, T. Akasaka; Carbon 43 (2005) 3124

150 M.T. Martinez, M.A. Callejas, A.M. Benito, M. Cochet, T. Seeger, A. Anson et al; Carbon 41 (2003) 2247

${ }^{151}$ P. Umek, A. Hassanien, M. Tokumoto, D. Mihailovic; Carbon 38 (2000) 1723

152 J.F. Colomer, P. Piedigrosso, A. Fonseca and J.B. Nagy; Synth. Met. 103 (1999) 2482

${ }^{153}$ E. Raymundo-Pinero, T. Cacciaguerra, P. Simon, F. Beguin; Chem. Phys. Lett. 412 (2005) 184

${ }^{154}$ T. Suzuki, K. Suhama, X. Zhao, S. Inoue, N. Noriyoshi, Y. Ando; Diam. Relat. Mater. 16 (2007) 1116

${ }^{155}$ A. Rasheed, J.Y. Howe, M.D. Dadmun, P.F. Britt; Carbon 45 (2007) 1072

${ }^{156}$ E. Raymundo-Pinero, D. Cazorla-Amoros, A. Linares-Solano, S. Delpeux, E. Frackowiak, K. Szostak et al; Carbon 40 (2002) 1614

${ }^{157}$ E. Raymundo-Pinero, P. Azais, T. Cacciaguerra, A. Linares-Solano, D. Cazorla-Amoros, F. Beguin, Carbon 43 (2005) 786

${ }^{158}$ F.H. Ko, C.Y. Lee, C.J. Ko, T.C. Chu; Carbon 43 (2005) 727

${ }^{159}$ C.M. Chen, M. Chen, F.C. Leu, S.Y. Hsu, S.C. Wang, S.C. Shi et al; Diam. Relat. Mater. 13 (2004) 1182

${ }^{160}$ C.M. Chen, M. Chen, Y.W. Peng, H.W. Yu, C.F. Chen; Thin Solid Films 498 (2006) 202

${ }^{161}$ M. Chen, H.W. Yu, J.H. Chen, H.S. Koo; Diam. Relat. Mater. 16 (2007) 1110

162 C.J. Ko, C.Y. Lee, F.H. Ko, H.L. Chen, T.C. Chu; Microelectron. Eng. 570 (2004) 73

${ }^{163}$ C. Li, D. Wang, T. Liang, X. Wang, J. Wu, X. Hu, J. Liang; Powder Technol. 142 (2004) 175

${ }^{164}$ M.S.P. Shaffer, X. Fan, A.H. Windle; Carbon 36 (1998) 1603

${ }^{165}$ Y. Ando, X. Zhao, H. Shimoyama; Carbon 39 (2001) 569

${ }^{166}$ M.R. Smith, S.W. Hedges, R. Lacount, D. Kern, N. Shah, G.P. Huffman et al, Carbon 41 (2003) 1221

167 S.R.C. Vivekchand, A. Govindaraj, M. Seikh, C.N.R. Rao, J. Phys. Chem. B 108 (2004) 6935

${ }^{168}$ Y. Wang, L. Gao, J. Sun, Y. Liu, S. Zheng, H. Kajiura. Phys. Lett. 432 (2006) 205

${ }^{169}$ T. Abatemarco, J. Stickel, J. Belfort, B.P. Frank, P.M. Ajayan, G. Belfort; J. Phys. Chem. B 103 (1999) 3534. 
${ }^{170}$ N. Tagmatachis, A. Zattoni, P. Reschiglian, M. Prato, Carbon 43 (2005) 1984.

171 K.B. Shelimov, R.O. Esenaliev, A.G. Rinzler, C.B. Huffman, R.E. Smalley; Chem.Phys.Lett. 282 (1998) 429

172 K.L. Lu, R.M. Lago, Y.K. Chen, M.L.H Green, P.J.F Harris, S.C. Tsang, Carbon 34 (1996) 814

${ }^{173}$ K.B. Shelimov, R.O. Esenaliev, A.G. Rinzler, C.B. Huffman, R.E. Smalley; Chem. Phys. Lett. 282 (1998) 429

${ }^{174}$ E. Farkas, M.E. Anderson, Z. Chen and A.G. Rinzler; Chem. Phys. Lett. 363 (2002) 111

${ }^{175}$ Y. Yang, L. Xie, Z. Chen, M. Liu, M. Zhu, T. Zhu,Z. Liu; Synth. Met. 155 (2005) 455

${ }^{176}$ G.S. Duesberg, W. Blau, H.J. Byene, J. Muster, M. Burghard, S. Roth; Synth. Met. 103 (1999) 2482

${ }^{177}$ J. Zhao, J.P. Lu, J. Han, C.K. Yang, Appl. Phys. Lett. 82 (2003) 3746

178 J. Chattopadhyay, I. Galeska, F. Papadimitrakopoulos, J. Am. Chem. Soc., 125 (2003) 3370

179 B.R. Azamian, J.J. Davis, K.S. Coleman, C.B. Bagshaw, M.L.H. Green, J. Am. Chem. Soc. 124 (2002) 12664

${ }^{180}$ R.J. Chen, Y. Zhang, D. Wang, H. Dai, J. Am. Chem. Soc. 123 (2001) 3838

${ }^{181}$ W. Huang, S. Taylor, K. Fu, D. Zhang, T.W. Hanks, A.M. Rao et al; Nano Lett. 2 (2002) 311

${ }^{182}$ M. Zheng, A. Jagota, E.D. Semke, B.A. Diner, R.S. McLean, S.R. Lustig; Nature Mater. 2 (2003) 338

${ }^{183}$ M.S. Strano, M. Zheng, A. Jagota, G.B. Onoa, D.A. Heller, P.W. Barone; Nano Lett. 4 (2004) 543

${ }^{184}$ M. Zheng, A. Jagota, M.S. Strano, A.P. Santos, P.W. Barone, S.G. Chou et al; 302 (2003) 1545

${ }^{185}$ H. Murakami, T. Nomura, N. Nakashima; Chem. Phys. Lett. 378 (2003) 481

${ }^{186}$ P.M. Ajayan, T.W. Ebbesen, T. Ichihashi, S. Iijima, K. Tanigaki, H. Hiura; Nature 362 (1993) 522

187 W. Xia, C. Jin, S. Kundu, M. Muhler; Carbon 47 (2009) 919

${ }^{188}$ M. Grujicic, G. Gao, A.M. Rao, T.M. Tritt, S. Nayak; Appl. Surf. Sci. 214 (2003) 289

${ }^{189}$ T. Savage, S. Bhattacharya, B. Sadanadan, J. Gaillard, T.M. Tritt, Y.P. Sun, et al; J. Phys. Conden. Mat. 15 (2003) 5915

${ }^{190}$ V. Datsyuk, M. Kalyva, K. Papagelis, J. Parthenios, D. Tasis, A. Siokou, et al; Carbon 46 (2008) 833

${ }^{191}$ K.J. Ziegler, Z. Gu, H. Peng, E.L. Flor, R.H. Hauge, R.E. Smalley J. Am. Chem. Soc. 127 (2005) 1541

192 Y.J. Kim, T.S. Shin, H.D. Choi, J.H. Kwon, Y.C. Chung, H.G. Yoon; Carbon 43 (2005) 23

${ }^{193}$ V. Datsyuk, M. Kalyva, K. Papagelis, J. Parthenios, D. Tasis, A. Siokou; Carbon 46 (2008) 833

194 F. Aviles, J.V. Cauich-Rodriguez, L. Moo-Tah, A. May-Pat, R. Vargas-Coronado; Carbon, 47 (2009) 2970

${ }^{195}$ P.J. Boul, J. Liu, E.T. Mickelson, C.B. Huffman, L.M. Ericson, I.W. Chiang, et al; Chem. Phys. Lett. 310 (1999) 367

${ }^{196}$ H. Peng, P. Reverby, V.N. Khabashesku, J.L. Margrave; Chem. Commun. (2003) 362

197 A. Gromov, S. Dittmer, J. Svensson, O.A. Nerushev, S.A. Perez-Garcia, L. LiceaJimenez et al; J. Mater. Chem. 15 (2005) 3334

${ }_{198}$ P.C. Ma, J.-K. Kim, B.Z. Tang; Carbon 44 (2006) 3232

${ }^{199}$ Y. Wang, Z. Iqbal, S.V. Malhotra; Chem. Phys. Lett. 402 (2005) 96. 
${ }^{200}$ N. Hu, G. Dang, H. Zhou, J. Jing, C. Chen; Mater. Lett. 61 (2007) 5285

${ }^{201}$ Y. Yao; W. Li, S. Wang D. Yan, X. Chen; Macromol. Rapid Commun. 27 (2006) 2019.

${ }^{202}$ A.M. Zhang, J L. Dong, Q.H. Xu, H.K. Rhee, X.L. Li; Catal. Today, 93-95 (2004) 347

${ }^{203}$ R. Puskas, A. Sapi, A. Kukovecz, Z. Konya; Top. Catal. 55 (2012) 865

${ }^{204}$ S. Dominguez-Dominguez, A. Berenguer-Murcia, B.K. Pradhan, A. Linares-Solano, D. Cazorla-Amoros; J. Phys. Chem. C 112 (2008) 3827

${ }^{205}$ L. Jiang, H. Gu, X. Xu, X. Yan; J. Mol. Catal. A Chem. 310 (2009) 144

${ }^{206}$ X.R. Ye, Y.H. Lin, C.M. Wang, M.H. Engelhard, Y. Wang, C.M. Wai; J. Mater. Chem. 14 (2004) 908

207 J.-P. Tessonnier, O. Ersen, G. Weinberg, C. Pham-Huu, D.S. Su, R. Schlögl; ACS Nano 3 (2009) 2081

${ }^{208}$ H. Ma, L. Wang, L. Chen, C. Dong, W. Yu, T. Huang, et al; Catal. Commun. 8 (2007) 452

${ }^{209}$ E. Castillejos, P.J. Deboutti, L. Roiban, A. Solhy, V. Martinez, Y. Kihn, et al. Angew. Chem. 121 (2009) 2567-2571

${ }^{210}$ T. Lu, E.M. Goldfield; J. Phys. Chem. C 112 (2008) 2654

${ }^{211}$ E.E. Santiso, M.K. Kostov, A.M. George, M. Buongiorno-Nardelli, K.E. Gubbins; Appl. Surf. Sci. 253 (2007) 5570

${ }_{212}$ M.D. Halls, H.B. Schlegel, J. Phys. Chem. B, 106 (2002) 1921

${ }^{213}$ W. Chen, L. Duan, D. Zhu; Environ. Sci. Technol. 41 (2007) 8295

${ }^{214}$ M. Kragulj, J. Trickovic, B. Dalmacija, A. Kukovecz, Z. Konya, J. Molnar et al; Chem. Eng. J. 225 (2013) 144

215 A.J. Brooks, H. Lim, J.E. Kilduff; Nanotechnology 23 (2012) 294008

${ }^{216}$ S. Gotovac, C.-M. Yang, Y. Hattori, K. Takahashi, H. Kanoh, K. Kaneko; J. Coll. Interf. Sci. 314 (2007) 18

${ }^{217}$ W. Liu, B. Wu, C. Cha; J. Electroanal. Chem. 476 (1999) 101

${ }^{218}$ R. Remias, A. Sapi, R. Puskas, A. Kukovecz, Z. Konya, I. Kiricsi; Chem. Phys. Lett. 482 (2009) 296

${ }^{219}$ M.S. Mohlala , X.-Y. Liu , J. M. Robinson , N.J. Coville; Organometallics 24 (2005) 972

${ }^{220}$ D.K. Smith, D.C. Lee, B.A. Korgel; Chem. Mater. (2006) 183356

221 A. Koshio, M. Shiraishi, Y. Kobayashi, M. Ishihara, Y. Koga, S. Bandow et al; Chem. Phys. Lett. 396 (2004) 410

${ }^{222}$ B. Xue, P. Chen, Q. Hong, J. Lin, K.L. Tan; J. Mater. Chem. 11 (2001) 2378

${ }^{223}$ A. Govindaraj, B.C. Satishkumar, M. Nath, C.N.R. Rao, Chem. Mater. 12 (2000) 202

${ }^{224}$ J.H. Bitter, M.K. van der Lee, A.G.T. Slotboom, A.J. van Dillen, K.P. de Jong; Catal. Lett. 89 (2003) 139

${ }^{225}$ X.-W. Wei, J. Xu, X.-J. Song, Y.-H. Ni, P. Zhang, C.-J. Xia, et al; Mater. Res. Bull 41 (2006) 92

${ }^{226}$ Y.-T. Kim, T. Mitani; J. Catal. 238 (2006) 394

${ }^{227}$ S. Komarneni, D. Li, B. Newalkar, H. Katsuki, A.S. Bhalla; Langmuir 18 (2002) 5959

${ }^{228}$ Z. Liu, J.Y. Lee, W. Chen, M. Han, L.M. Gan; Langmuir 20 (2004) 181

${ }^{229}$ C. Liu, X. Wu, T. Klemmer, N. Shukla, X. Yang, D. Weller; J. Phys. Chem. B, 108 (2004) 6121

${ }^{230}$ W.X. Chen, J.Y. Lee and Z. Liu, Chem. Commun. (2002) 2588

${ }^{231}$ H.C. Choi, M. Shim, S. Bangsaruntip H. Dai; J. Am. Chem. Soc 124 (2002) 9058

${ }^{232}$ L. Qu, L. Dai; J. Am. Chem. Soc 127 (2005) 10806

233 A.E. Fischer, K.A. Pettigrew, D.R. Rolison, R.M. Stroud, J.W. Long; Nano Lett. 7 (2007) 281

${ }^{234}$ Y. Okinaka, M. Hoshino; Gold Bull. 31 (1998) 3 
${ }^{235}$ H.W. Zhu, C.L. Xu, D.H. Wu, B.Q. Wei, R. Vajtai, P.M. Ajayan; Science 296 (2002) 884

${ }^{236}$ G. Liu, Y. Zhao, K. Deng, Z. Liu, W. Chu, J. Chen et al; Nano Lett. 8 (2008) 1071

${ }^{237}$ W. Ma, L. Liu, R. Yang, T. Zhang, Z. Zhang, L. Song, et al. Adv. Mater. 21 (2009) 603

${ }^{238}$ M. Zhang, K.R. Atkinson, R.H. Baughman; Science 306 (2004) 1358

${ }^{239}$ X. Zhang, Q. Li, Y. Tu, Y. Li, J.Y. Coulter, L. Zheng et al; Small 3 (2007) 244

${ }^{240}$ M. Motta, A. Moisala, I. A. Kinloch, A. H. Windle; Adv. Mater. 19 (2007) 3721

${ }^{241}$ K. Liu, Y. Sun, R. Zhou, H. Zhu, J. Wang, L. Liu, et al; Nanotechnology 21 (2010) 045708

${ }^{242}$ Y.L. Li, I.A. Kinloch, A.H. Windle; Science 304 (2004) 276.

${ }^{243}$ H.W. Zhu, C.L. Xu, D.H. Wu, B.Q. Wei, R. Vajtai, P.M. Ajayan; Science 296 (2002) 884

${ }^{244}$ Y.L. Li, I.A. Kinloch, A.H. Windle; Science 304 (2004) 276.

${ }^{245}$ X.H. Zhong, Y.L. Li, Y.K. Liu, X.H. Qiao, Y. Feng, J. Liang; Adv. Mater. 22. (2009) 692

${ }^{246}$ W. Ma, L. Song, R. Yang, T. Zhang, Y. Zhao, L. Sun; Nano Lett. 7 (2007) 2307

${ }^{247}$ W. Zhou, X. Bai, E. Wang, S. Xie; Adv. Mater. 21 (2009) 4565

${ }^{248}$ X. Zhang, K. Jiang, C. Feng, P. Liu, L. Zhang, J. Kong et al; Adv. Mater. 18 (2006) 1505

${ }^{249}$ M. Zhang, S. Fang, A.A. Zakhidov, S.B. Lee, A.E. Aliev, C.D. Williams et al; Science 309 (2005) 1215

${ }^{250}$ J. Di, D. Hu, H. Chen, Z. Yong, M. Chen, Z. Feng. et al. ACS Nano 6 (2012) 5457

${ }^{251}$ K. Liu, Y. Sun, L. Chen, C. Feng, X. Feng, K. Jiang, Y. Zhao, S. Fan, Nano Lett. 8 (2008) 700.

${ }^{252}$ X. Li, L. Zhang, X. Wang, I. Shimoyama, X. Sun, W.S. Seo; J. Am. Chem. Soc. 129 (2007) 4890.

${ }^{253}$ M.C. LeMieux, M. Roberts, S. Barman, Y.W. Jin, J.M. Kim, Z. Bao; Science 321 (2008) 101

${ }^{254}$ H.Z. Geng, K.K. Kim, K.P. So, Y.S. Lee, Y. Chang, Y.H. Lee; J. Am. Chem. Soc. 129 (2007) 7758

${ }^{255}$ R. Duggal, F. Hussain, M. Pasquali, Adv. Mater.18 (2006) 29

256 J. Liu, A.G. Rinzler, H. Dai, J.H. Hafner, R.K. Bradley, P.J. Boul, et al; Science 280 (1998) 1253

${ }^{257}$ Z. Wu, Z. Chen, X. Du, J.M. Logan, J. Sippel, M. Nikolou, et al; Science 305 (2004) 1273

${ }^{258}$ D. Wang, P. Song, C. Liu, W. Wu, S. Fan; Nanotechnology 19 (2008) 075609

259 W.Z. Li, S.S. Xie, L.X. Qian, B.H. Chang, B.S. Zou, W.Y. Zhou, et al; Science 274 (1996) 1701

${ }^{260}$ Z.F. Ren, Z.P. Huang, J.W. Xu, J.H. Wang, P. Bush, M.P. Siegal et al; Science 282 (1998) 1105

${ }^{261}$ S. Fan, M.G. Chapline, N.R. Franklin, T.W. Tombler, A.M. Cassell, H. Dai; Science 283 (1999) 512

${ }^{262}$ H. Sugime, S. Noda, S. Maruyama, Y. Yamaguchi; Carbon 47 (2009) 234

${ }^{263}$ K. Hasegawa, S. Noda; Appl. Phys Express 3 (2010) Num. 4

${ }^{264}$ K. Hata, D.N. Futaba, K. Mizuno, T. Namai, M. Yumura, S. Iijima; Science 306 (2004) 1362

${ }^{265}$ H. Liu, Y. Zhang, R. Li, X. Sun, F. Wang, Z. Ding; Appl. Surf. Sci. 256 (2010) 4692

${ }^{266}$ W.D. Cho, M. Schulz, V. Shanot; Carbon 72 (2014) 264

${ }^{267}$ S. Noda, K. Hasegawa, H. Sugime, K. Kakehi, Z. Zhang, S. Maruyama; Jpn. J. Appl. Phys Part 2; 46 (2007) L399 Num 17-19

${ }^{268}$ C.R. Oliver, E.S. Polsen, E. R. Meshot, S. Tawfick, S. J. Park, M. Bedewy et al; ACS Nano 7 (2013) 3565

${ }^{269}$ S. Talapatra, S. Kar, S.K. Pal, R. Vajtai, L. Ci, P. Victor et al; Nat. Nanotechnol. 1 (2006) 112. 
${ }^{270}$ Y.J. Jung, S. Kar , S. Talapatra, C. Soldano, G. Viswanathan, X. Li et al. Nano Lett. 6 (2006) 413

${ }^{271}$ L. Ci, S. M. Manikoth, X. Li, R. Vajtai, P. M. Ajayan, Adv. Mater. 19 (2007) 3300

${ }^{272}$ M. De Volder, S.H. Tawfick, S.J. Park, D. Copic, Z.Z. Zhao, W. Lu, et al; Adv. Mater. 22 (2010) 4384

${ }^{273}$ X. Gui, J. Wei, K. Wang, A. Cao, H. Zhu, Y. Jia, et al; Adv. Mater. 22 (2010) 617.

${ }^{274}$ V.N. Khabashesku, Z. Gu, B Brinson, J.L. Zimmerman, J.L. Margrave; J. Phys. Chem. B, 106 (2002) 11155

${ }^{275}$ J.L. Li, G.Z. Bai, J.W. Feng, W. Jiang ; Carbon 43 (2005) 2649

${ }^{276}$ Y. Sato, M. Ootsubo, G. Yamamoto, G. Van Lier, M. Terrones, S. Hashiguchi et al; ACS Nano 2 (2008) 348

${ }^{277}$ M.B. Bryning, D.E. Milkie, M.F. Islam, L.A. Hough, J.M. Kikkawa, A.G. Yodh; Adv. Mater. 19 (2007) 661

${ }^{278}$ J. Zou, J. Liu, A.S. Karakoti, A. Kumar, D. Joung, Q. Li et al; ACS Nano 4 (2010) 7293

${ }^{279}$ K.H. Kim, Y. Oh, M.F. Islam; Nature Nanotechnol. 7 (2012) 562

${ }^{280}$ M.O. Nutt, K.N. Heck, P. Alvarez, M.S. Wong; App. Catal. B 69 (2006) 115

${ }^{281}$ N. Yao, V. Lordi, S.X.C. Ma; J. Mater. Res. 13,(1998) 2432

${ }^{282}$ E. Dujardin, T.W. Ebbesen, A. Krishnan, M.M.J. Treacy; Adv. Mater. 10 (1998) 611 